\title{
LES FONCTIONS DE TYPE POSITIF ET LA THEORIE DES GROUPES
}

\author{
PAR \\ ROGER GODEMENT

\section{INTRODUCTION}

La théorie des groupes topologiques, bien que de création récente, constitue aujourd'hui une branche importante des mathématiques; elle englobe des théories comme: la mesure de Haar, la théorie du produit de composition, les séries et intégrales de Fourier, les fonctions presque-périodiques, les groupes d'opérateurs unitaires, et en partie, la théorie du potentiel, la théorie ergodique et la topologie algébrique.

On connait les principales étapes de son développement: l'extension de la théorie des séries de Fourier aux groupes de Lie compacts par F. Peter et $\mathrm{H}$. Weyl; la démonstration de l'existence d'un mesure invariante par A. Haar; la théorie des fonctions presque périodiques due à J. von Neumann; la théorie de la dualité pour les groupes abéliens par L. Pontrjagin et A. R. van Kampen; enfin l'année 1940 vit apparaitre le traité fondamental d'André Weil qui, outre une systématisation à peu près parfaite des résultats antérieurs, apportait une construction complète de l'analyse harmonique sur les groups abéliens.

A partir de cette date, c'est avant tout à l'école de Moscou, représentée particulièrement, semble-t-il, par D. Raīkov, I. Gelfand, et M. Neumark, que l'on doit les progrès les plus considérables. Alors que jusqu'en 1940 tous les travaux concernaient les représentations unitaires de dimension finie, Gelfand et Raïkov, dans un mémoire paru en 1943, étudient systématiquement les représentations unitaires de dimension quelconque (finie ou non) et prouvent l'existence, sur tout groupe localement compact, d'un système complet de représentations unitaires irréductibles-résultat qui contient tous ceux connus jusqu'alors dans cette ordre d'idées et qui, au surplus, n'avait jamais été demontré dans les cas classiques-celui du groupe de Lorentz par exemple.

Le but du présent travail est de poursuivre les recherches précédentes. Les principaux résultats en sont: (1) l'extension à un groupe quelconque des théorèmes d'approximation par ce qui remplace les "polynômes trigonométriques": résultat qui contient donc, en particulier, le théorème de Peter et Weyl (qui est relatif à un groupe compact); (2) l'extension à un groupe quelconque des propriétés "spectrales" des fonctions de type positif, et même d'autres classes de fonctions (dans cette voie, c'est le remarquable résultat publié récemment par A. Beurling qui m'a guidé) ainsi que l'extension du

Presented to the Society, October 25, 1947; received by the editors November 7, 1946. 
théorème de Bochner, et de quelques propriétés des spectres; (3) une étude complète du "spectre ponctuel" d'une représentation unitaire quelconque, et des propriétés correspondantes des fonctions de type positif (ce qui contient une partie de la théorie des fonctions presque-périodiques); (4) une étude systématique des fonctions de carré sommable, faite au point de vue du produit de composition $\left({ }^{1}\right)$.

D'autre part, un article qui sera publié séparément contiendra un éxposé complet et direct de la théorie des groupes abéliens, basé lui aussi sur les méthodes de Gelfand et Raïkov, et conçu de manière à constituer une éventuelle base de départ vers une extension de l'analyse harmonique aux groupes non abéliens.

Le lecteur constatera que je pose aussi un certain nombre de problèmes que je n'ai pu résoudre dans le cas général, et qui semblent, pour l'instant, arrêter quelque peu les progrès qu'on est en droit d'espérer.

Il m'a paru d'autre part impossible de ne pas réexposer complètement les travaux de Gelfand et Raïkov, tout d'abord parce; que en les supposant connus, $\mathbf{j}$ 'aurais compromis fâcheusement l'unité de ce travail ; ensuite parce que, ayant été publiés en russe, ils sont peut-être d'un accès quelque peu difficile à la majorité des malheureux occidentaux; enfin parce que, n'ayant eu connaissance moi-même du mémoire en question qu'en février 1946, j'ai passé un certain nombre de mois à retrouver une partie des résultats qu'il contient, comme on le constatera en lisant les Notes que j'ai publiées aux Comptes rendus entre juillet 1945 et janvier 1946. Cette dernière raison m'amène plus généralement à faire des réserves en ce qui concerne l'originalité éventuelle de mes résultats, une grande partie des publications russes étant actuellement introuvable en France.

En ce qui concerne la terminologie et les méthodes adoptées, ce sont en principe celles de A. Weil et de $\mathrm{N}$. Bourbaki. J'ai cependant repris complètement la théorie du produit de composition, en insistant plus que ne le fait A. Weil sur le rôle du facteur $\rho(s)$ (noté $\Delta(s)$ par A. Weil et $l(s)$ par Gelfand et Raikov) de la mesure de Haar. La plupart des résultats de cet exposé préliminaire sont du reste connus, et se trouvent soit dans le livre d'A. Weil, soit dans le mémoire cité d'H. Cartan.

Qu'il me soit permis maintenant d'exprimer à Mr Henri Cartan toute la reconnaissance que je lui dois pour la façon dont il m'a aidé à poursuivre ces recherches. Sa contribution ne s'est pas bornée à revoir ligne par ligne les rédactions successives de cet article et à les améliorer considérablement; il m'a souvent mis sur la voie de nouveaux résultats-par exemple en ce qui concerne le spectre d'une fonction de type positif-et m'a permis par ses remarques, d'éclaircir beaucoup d'idées qui, sans cela, fussent restées quelque peu nuageuses. Il m'a aussi définitivement conduit à penser que les mathé-

(1) H. Cartan et R. Godement, Analyse harmonique et théorie de la dualite dans les groupes abeliens localement compacts, Ann. Ecole Norm. 
matiques ne sont pas nécessairement étrangères aux soucis esthétiques (ceci dit sans préjuger du résultat obtenu, qu'on devra m'attribuer s'il est mauvais).

Enfin, je remercie sincèrement $M M$. Elie Cartan et Jean Favard, qui ont accepté de se joindre à M: Henri Cartan pour constituer le jury auquel cette thèse a été soumis; $M$. Elie Cartan, au surplus, a bien voulu présenter mes Notes à l'Académie des Sciences-ce dont je lui suis également infiniment reconnaissant.

\section{INDEX DES DEFINITIONS ET DES NOTATIONS $\left({ }^{2}\right)$}

Caractéristique (fonction.......... 7

contenue (structure unitaire) . . . . . . 5

convergence $C$-faible............ 22

convergence étroite.............. 1

convergence faible (dans $L$ )........ 13

convergence $\mathfrak{H}$-faible............. 22

convergence vague. ............. 1

Disjointes (fonctions de t.p.)........ 12

disjointe (partie) .............. 12

dual (d'un espace de Banach) ........ 13

Elémentaire (fonction) $\ldots \ldots \ldots \ldots \ldots 11$

ergodique (fonction) $\ldots \ldots \ldots \ldots \ldots \ldots 23$

extrémal (point)................ 13

Irréductible (structure unitaire) ....... 5

isomorphes (structures unitaires) ...... 5

Mesure de Radon.................. 1

Norme (d'une mesure)............ 1

Partition (d'une fonction de t.p.)...... 10

polynóme trigonométrique.......... 15

produit de composition (mesures)...... 2

produit de composition (fonctions) ..... 3

produit tensoriel. ............... 20

Représentation unitaire........... 5

régularisation (d'une mesure) ........ 4

Sommable sur tout compact......... 3 spectre (d'une fonction de t.p.). . . . . 15

spectre (d'une fonction qcq.)........ 18

structure unitaire............... 5

structure unitaire simple........... 5

Type positif (fonctions) ........... 8

type positif (mesures) ............. 9

Unite (dans $\left.L^{2}\right) \ldots \ldots \ldots \ldots \ldots \ldots \ldots 29$

$L_{0} ; L_{0}^{+} ; L ; L_{\infty} ;\|\mu\|_{\mathrm{h}} \ldots \ldots \ldots \ldots \ldots \ldots .1$

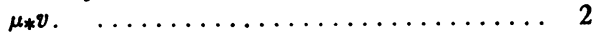

$d x ; \rho(x) ; L^{p} ;\|f\|_{p} ; L^{\infty} ;\|f\|_{\infty} ; f_{*} g \ldots \ldots \ldots, 3$

$U_{x} ;\left\{\mathcal{H C}, U_{x}\right\} ;\left\{\mathcal{F C}, U_{x}, X\right\} \ldots \ldots \ldots \ldots .5$

$T_{\mu} \ldots \ldots \ldots \ldots \ldots \ldots \ldots \ldots \ldots \ldots$

$C^{\infty} ; f_{s} ; V_{\phi} ;(\psi, \theta)_{\phi} ; \mathcal{F C}_{\phi} ; H_{\phi} \ldots \ldots \ldots \ldots \ldots 7$

$f^{\sim} ; \mu^{\phi} ;(f, g\rangle_{\mu} ; p ; \Phi ; \mu \ll{ }_{\nu} \ldots \ldots \ldots \ldots . \quad 9$

$\phi \ll \psi \ldots \ldots \ldots \ldots \ldots \ldots \ldots \ldots \ldots \ldots$

$E ; E^{\prime} ;\|f\| ;(f, \phi) ; \Phi_{0} \ldots \ldots \ldots \ldots \ldots 14$

$A_{\phi} ; \bar{A}_{\phi} \ldots \ldots \ldots \ldots \ldots \ldots \ldots \ldots \ldots 15$

$G \backslash ; \Phi ; \sigma_{A} ; \mathfrak{F} \uparrow \ldots \ldots \ldots \ldots \ldots \ldots \ldots 16$

$\Gamma ; \Lambda_{\infty} ; \mathscr{H}_{0}^{+} ; \phi_{\mu} \ldots \ldots \ldots \ldots \ldots \ldots 17$

$X \wedge X^{\prime} ; \mathfrak{H} \wedge \mathfrak{H}^{\prime} \ldots \ldots \ldots \ldots \ldots \ldots \ldots 20$

$\left\{\mathcal{C}_{\phi}, U_{x}\right\} \wedge\left\{\mathcal{H}_{\phi}, U_{x}\right\} \ldots \ldots \ldots \ldots \ldots 21$

$M_{i}[\phi(t)] ; M(\phi) \ldots \ldots \ldots \ldots \ldots \ldots \ldots 23$

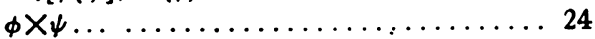

$U_{s} ; V_{\varepsilon} ; P^{2} ; \mathbb{P}^{2} ;[f, g] ; A_{\mu} ; P^{I} ; \mathcal{P}^{1} ; A_{\phi} \ldots \ldots 26$

$f^{\prime}(x)=f\left(x^{-I}\right) /(\rho(x))^{1 / 2} \ldots \ldots \ldots \ldots \ldots 28$

\section{RAPPEL DE NOTIONS PRELIMINAIRES: THEORIES DE}

\section{LA MESURE ET DU PRODUIT DE COMPOSITION}

1. Mesures de Radon-convergence des mesures $\left({ }^{(2)}\right) G$ étant un espace topologique localement compact (=localement bicompact au sens d'Alexandroff-Hopf) on désignera par $L_{0}$ l'ensemble des fonctions numériques réelles à valeurs finies sur $G$, continues, et nulles en dehors de parties compactes de $G$. $L_{0}^{+}$étant l'ensemble des $f \geqq 0$ de $L_{0}$, une mesure de Radon positive sur $G$ sera une fonctionnelle linéaire $\mu(f)$ définie sur $L_{0}$ et positive sur $L_{0}^{+}$. On la notera aussi

(2) Les numeros renvoient aux paragraphes du texte.

(8) La methode suivie ici est celle de H. Cartan, Sur les fondements de la théorie du potentiel (Bull. Soc. Math. France t. 69 (1941) pp. 71-96). 


$$
\mu(f)=\int f(x) d \mu(x)=\int f d \mu .
$$

On définira de manière évidente les mesures complexes (de la forme $\mu_{1}-\mu_{2}$ $+i\left(\mu_{3}-\mu_{4}\right)$ où les $\mu_{p}$ sont positives) ainsi que la valeur de (I 1$)$ pour $f=g+i h$ $\left(g, h \in L_{0}\right)$; l'ensemble de ces $f$ sera noté $L$. Soit $\mu$ une mesure positive; soit $g(x)$ une fonction positive et semi-continue inférieurement sur $G$, pouvant prendre la valeur $+\infty$. On posera

$$
\mu(g)=\int g d \mu=\sup \int f d \mu
$$

pour $f \in L_{0}^{+}$et $f(x) \leqq g(x)$. De même, si $h(x)$ est positive, semi-continue supérieurement, à valeurs finies sur $G$, et nulle en dehors d'un compact, on posera

$$
\mu(h)=\int h d \mu=\inf \int f d \mu \quad(<+\infty)
$$

pour $f \in L_{0}^{+}$et $f(x) \geqq h(x)$.

Désignens par $\Im_{0}^{+}$et $\mathfrak{S}_{0}^{+}$les deux classes de fonctions qu'on vient de définir. Une fonction $f(x)$ positive (et pouvant prendre la valeur $+\infty$ ) sera dite sommable pour $\mu \mathrm{si}$

$$
\inf \int g d \mu=\sup \int h d \mu
$$

pour $g \in \Im_{0}^{+}, h \in \Im_{0}^{+}$et $h(x) \leqq f(x) \leqq g(x)$, la valeur commune des deux membres étant par définition

$$
\mu(f)=\int f d \mu=\int f(x) d \mu(x) .
$$

Une $f(x)$ réelle sera sommable si

$$
f^{+}(x)=\sup [f(x), 0], \quad f^{-}(x)=\sup [-f(x), 0]
$$

le sont; et on posera

$$
\int f d \mu=\int f^{+} d \mu-\int f^{-} d \mu
$$

Enfin une $f(x)$ complexe le sera si $\Re f(x)$ et $\Im f(x)$ le sont. Posons, pour $f(x)$ bornée et continue sur $G$,

$$
\|f\|_{\infty}=\sup _{x \in G}|f(x)|
$$

Une $\mu$ réelle ou complexe sera de normie finie (ou bornée) si 


$$
\|\mu\|_{1}=\sup \left|\int f d \mu\right| \operatorname{pour} f \in L,\|f\|_{\infty} \leqq 1 \text { est }<+\infty .
$$

C'est toujours le cas si $\mu$ est "portée par un compact" $K \subset G$, c'est à dire si

$$
\int f d \mu=0 \text { pour toute } f \in L \text { nulle sur } K \text {. }
$$

Si $\mu$ est bornée, toute $\phi(x)$ continue et bornée sur $G$ est sommable; de plus

$$
\left|\int \phi d \mu\right| \leqq\|\phi\|_{\infty}\|\mu\|_{1}
$$

et, si $\mu$ est positive:

$$
\|\mu\|_{1}=\int d \mu(x)=\mu(1) .
$$

La propriété fondamentale des mesures bornées est la suivante: désignons par $L_{\infty}$ l'ensemble des fonctions numériques $f(x)$ bornées et continues sur $G$, et telles que pour tout $\epsilon>0$ l'ensemble des $x \in G$ avec $|f(x)| \geqq \epsilon$ soit compact; munissons $L_{\infty}$ de la norme $\|f\|_{\infty}$ définie ci-dessus. Alors, $L_{\infty}$ est un espace de Banach, et l'ensemble des mesures bornées sur $G$ est identique au $d u a l$ de $L_{\infty}$; de plus, la norme $\|\mu\|_{1}$ définie ci-dessus est aussi celle de $\mu(f)$ considéré comme fonctionnelle linéaire bornée sur $L_{\infty}$.

Dans l'ensemble des mesures de Radon réelles, on peut introduire une relation d'ordre qu'on notera $\mu \leqq \nu$ et qui signifiera que la mesure $\nu-\mu$ est positive. L'ensemble de ces mesures est réticulé: pour toute $\mu$ on peut définir $|\mu|$ et

$$
\mu^{+}=\sup (\mu, 0), \quad \mu^{-}=\sup (-\mu, 0) \quad\left(|\mu|=\mu^{+}+\mu^{-}\right)
$$

par

$$
\int f d \mu^{+}=\sup \int g d \mu \quad \text { pour } g \in{L_{0}}^{+}, g \leqq f,
$$

pour toute $f \in L_{0}^{+}$. Il est facile de voir que $\mu=\mu^{+}-\mu^{-}$. Si $\mu$ et $\nu$ sont réelles, $\sup (\mu, \nu)$ existe et est définie par

$$
\sup (\mu, \nu)=\nu+(\mu-\nu)^{+}=\mu+(\nu-\mu)^{+} .
$$

L'ensemble des $\mu \geqq 0$ forme ainsi un "domaine fondamental" au sens de F. Riesz( $\left.{ }^{4}\right)$, et même un peu plus, puisque c'est l'ensemble des fonctionnelles linéaires et positives sur le domaine fondamental $L_{0}^{+}$.

$\left({ }^{4}\right)$ F. Riesz, Sur quelques notions fondamentales de la théorie des opérations linéaires, Ann. of Math. t. 41 (1940). 
Introduisons maintenant, dans l'ensemble des mesures de Radon sur $G$, une topologie dont on aura constamment à se servir par la suite. On dira qu'une mesure $\mu$ variable converge vaguement $\left(^{(5)}\right.$ vers une mesure $\mu_{0} \mathrm{si}$, pour toute $f \in L, \int f d \mu$ tend vers $\int f d \mu_{0}$. Cette topologie correspond aux voisinages $\mathfrak{B}\left(\mu_{0} ; \epsilon ; f_{1}, \cdots, f_{n}\right)$ obtenus en prenant les $\mu$ qui vérifient

$$
\left|\int f_{i} d \mu-\int f_{i} d \mu_{0}\right| \leqq \epsilon \quad \text { pour } f_{i} \in L .
$$

L'ensemble des $\mu$ devient ainsi un espace vectoriel topologique. La proposition suivante est facile à démontrer :

LeMme 1. (a) L'ensemble des mesures de Radon de norme $\leqq 1$ est compact pour la topologie vague.

(b) L'ensemble des mesures de Radon positives majorées par une mesure fixe est compact pour la topologie vague.

La partie (a) du Lemme 1 est une propriété classique - et triviale-des espaces de Banach, si l'on observe que: dans l'ensemble des $\mu$ de norme $\leqq 1$, la convergence vague n'est autre que la convergence faible dans le dual de $L_{\infty}$.

A côté de la convergence vague, on introduira la convergence étroite définie dans l'ensemble des mesures de norme bornée:

On dira qu'un filtre $\phi$, défini sur cet ensemble, converge étroitement vers $\mu_{0}$ si les conditions suivantes sont remplies:

(a) $\phi$ converge vaguement vers $\mu_{0}$;

(b) à tout nombre $\epsilon>0$ correspondent un compact $K \subset G$ et un élément $A \in \phi$ tels que, pour toute $\mu \in A$, la restriction de $\mu$ à l'adhérence de $G-K$ ait une masse totale inférieure à $\epsilon$.

Dans le cas où les $\mu$ sont toutes positives, on peut remplacer la condition (b) par la suivante, en apparence plus faible:

(b') la masse totale de $\mu$ tend vers celle de $\mu_{0}$.

LeMme 2. Pour qu'une $\mu$ bornée converge étroitement vers $\mu_{0}$, il faut et il suffit que $\int \phi d \mu$ tende vers $\int \phi d \mu_{0}$ pour toute fonction continue et bornée $\phi$ sur $G$.

Il est clair que, pour des $\mu$ portées par un compact fixe $K$ (donc en particulier si $G$ est compact), la convergence vague équivaut à la convergence étroite.

De plus, si on note $L_{K}$ l'espace des $f(x)$ continues sur $K$, normé par

$$
\|f\|_{K}=\sup _{x \in K}|f(x)|
$$

le dual de $L_{K}$ n'est autre que l'ensemble $\mathfrak{M}_{K}$ des mesures portées par $K$; sur

(5) Nous employons ici, comme dans toute la suite, une terminologie "naive"; mais il est indispensable pour la justifier d'employer les filtres (N. Bourbaki, Topologie génerale, chap. 1, p. 20) ou une méthode équivalente. Précisons que nous ne supposons jamais la séparabilité des espaces envisagés, ni aucune autre propriété analogue. 
$\mathfrak{M}_{K}$, la topologie vague (ou étroite) coincide avec la topologie faible bien connue dans la théorie des espaces de Banach. Cette remarque, conjuguée avec le classique théorème de Hahn-Banach, conduit au

Lemme 3. Si $G$ est compact, et si $D$ est un ensemble linéaire de fonctions continues sur $G$, une condition necessaire et suffisante pour que toute fonction continue sur $G$ soit limite uniforme de fonctions de $D$ est que

$$
\int f d \mu=0 \quad \text { pour toute } f \in D
$$

entraine $\mu=0$.

Si $G$ n'est pas compact, l'adjonction( $\left.{ }^{(}\right)$d'un "point à l'infini" à $G$ permet de le considérer comme plongé dans un compact $G$. Alors le Lemme 3 donne sans difficulté le

Lemme $3^{\prime}$. Pour qu'un ensemble linéaire $D C L_{\infty}$ soit dense dans $L_{\infty}$ au sens de la convergence uniforme, il faut et il suffit que toute $\mu$ bornée sur $G$ vérifiant

$$
\int f d \mu=0 \quad \text { pour toute } f \in D
$$

soit identiquement nulle.

2. Composition des mesures de Radon. Tout d'abord, soient $\mu$ et $\nu$ deux mesures $\geqq 0$ sur les espaces localement compacts $E$ et $F$.

Dans l'espace produit $E \times F$, considérons les fonctions de la forme

$$
g(x, y)=\sum_{i} e_{i}(x) f_{i}(y) \quad(x \in E, y \in F)
$$

où $e_{i}$ et $f_{i}$ appartiennent respectivement à $L_{E}$ et $L_{F}$; il est clair que $g \in L_{E \times \boldsymbol{r}}$. De plus( $\left.{ }^{7}\right)$ : toute $h \in L_{E \times F}$ nulle en dehors d'un compact de la forme $A \times B$ ( $A$ compact $C E, B$ compact $C F$ ) est limite, uniforme sur $E \times F$, de fonctions de la forme précédente avec $e_{i} \in L_{E}, f_{i} \in L_{F}$ et: $e_{i}$ nulles en dehors d'un compact convenable fixe $A^{\prime} \supset A$ et $f_{i}$ nulles en dehors d'un compact convenable fixe $B^{\prime} \supset B$.

Ces remarques permettent de voir que la fonctionnelle

$$
\lambda(g)=\sum \mu\left(e_{i}\right) \cdot \nu\left(f_{i}\right)
$$

est prolongeable à tout $L_{E \times F}$ d'où la mesure produit qu'on notera

$$
\iint g(x, y) d \lambda(x, y)=\iint g(x, y) d \mu(x) d \nu(y) .
$$

(") N. Bourbaki, loc. cit. p. 65 ("Théorème d'Alexandroff").

(7) J. Dieudonné. C. R. Acad. Sci. Paris t. 205 (1937) p. 593. 
De plus on a le classique

LeMme 4 (Lebesgue-Fubini). Si $\phi(x, y)$ est sommable sur $E \times F$ pour la mesure produit, alors

$$
e(x)=\int \phi(x, y) d \nu(y)
$$

existe pour presque tout $x \in E\left({ }^{7}\right)$ et est $\mu$-sommable; de même

$$
f(y)=\int \phi(x, y) d \mu(x)
$$

existe pour presque tout $y \in F\left({ }^{8}\right)$ et est $\nu$-sommable; enfin on a

$$
\iint \phi(x, y) d \mu(x) d \nu(y)=\left\{\begin{array}{l}
\int d \mu(x) \int \phi(x, y) d \nu(y), \\
\int d \nu(y) \int \phi(x, y) d \mu(x) .
\end{array}\right.
$$

Ceci étant, et si l'on a un groupe $G$ localement compact, on dira que le produit de composition de deux mesures positives $\mu, \nu$ existe si, pour toute $f \in L$, la fonction $f(x \cdot y)$ est sommable sur $G \times G$ pour la mesure produit, $\lambda=\mu * \nu$ étant alors défini par

$$
\int_{G} f(z) d \lambda(z)=\iint_{G \times G} f(x y) d \mu(x) d \nu(y) .
$$

Il est à peu près évident que $\mu * \nu$ existe dès que $\mu$ (ou $\nu$ ) est portée par un compact; que si $\mu$ et $\nu$ sont portées par les compacts $A$ et $B, \mu * \nu$ est portée par le compact $A \cdot B$; qu'enfin $\mu * \nu$ existe toujours si $\mu$ et $\nu$ sont de masses totales finies, auquel cas on a

$$
\|\mu * \nu\|_{1} \leqq\|\mu\|_{1} \cdot\|\nu\|_{1} .
$$

Bien entendu, on peut définir aussi $\mu * \nu$ pour des mesures complexes-en se ramenant à des mesures positives.

Lemme 5. (a) Si $\mu$ converge vaguement vers $\mu_{0}$, et si $\nu_{0}$ est portée par un compact, $\mu * \nu_{0}$ et $\nu_{0} * \mu$ convergent vaguement vers $\mu_{0} * \nu_{0}$ et $\nu_{0} * \mu_{0}$.

(b) Si $\mu$ converge vaguement vers $\mu_{0}$ en restant portée par un compart fixe et si $\nu_{0}$ est quelconque, $\mu * \nu_{0}$ et $\nu_{0} * \mu$ convergent vaguement vers $\mu_{0} * \nu_{0}$ et $\nu_{0} * \mu_{0}$.

(c) Si $\mu$ et $\nu$, portées par un compact fixe, convergent vaguement vers $\mu_{0}$

(8) "Presque tout $x^{n}$ s'applique à la mesure $\mu$; "presque tout $y$ " à la mesure $\nu$. Rappelons qu'une propriété a lieu "preșque partout relätivement à $\mu^{\text {" }}$ si les $x$ qui ne la vérifient pas constituent un ensemble $A$ dont la fonction caractéristique $\phi_{A}(x)$ est $\mu$-sommable et vérifie $\mu(A) \equiv \int_{\phi_{A}}(x) d \mu(x)=0$. 
et $\nu_{0}$, alors $\mu * \nu$ converge vaguement vers $\mu_{0} * \nu_{0}$.

3. Composition des fonctions. On sait $\left({ }^{9}\right)$ que, parmi les mesures de Radon positives sur le groupe $G$, il en existe une (et une seule à un facteur constant près) qui vérifie

$$
\int f\left(s^{-1} x\right) d \mu(x)=\int f(x) d \mu(x)
$$

pour toute $f \in L$ et tout $s \in G$. C'est la mesure de Haar invariante a gauche; on la notera $d x$.

On définirait de même une mesure invariante à droite. Si $s$ est un élément donné de $G, \int f\left(x s^{-1}\right) d x$ est aussi une fonctionnelle linéaire sur $L_{0}$, positive sur $L_{0}^{+}$, et invariante à gauche: d'après l'unicité de la mesure de Haar, il existe donc un facteur $\rho(s)>0$ tel qu'on ait

$$
\int f\left(x s^{-1}\right) d x=\rho(s) \int f(x) d x
$$

pour toute $f \in L$. Bien entendu $\rho(s)$ est une fonction continue et vérifie $\rho(s t)=\rho(t s)=\rho(s) \rho(t)$. Il est facile par ailleurs de voir que, pour toute $f \in L$ :

$$
\int f\left(x^{-1}\right) \frac{d x}{\rho(x)}=\int f(x) d x .
$$

Bien entendu ces égalités sont valables aussi pour toute $f(x)$ sommable pour $d x$. $G$ est dit unimodulaire si $\rho(s) \equiv 1$; c'est notamment le cas si $G$ est abélien ou compact. On dira qu'une $\phi(x)$ est sommable sur tout compact si $\phi(x) f(x)$ est sommable pour $d x$ quel que soit $f \in L$. Alors, l'expression

$$
\mu(f)=\int f(x) \phi(x) d x
$$

définit une mesure qu'on notera $\phi(x) d x$; celle-ci sera de masse totale finie si $\phi$ est sommable pour $d x$; on posera alors

$$
\|\phi\|_{1}=\int|\phi(x)| d x
$$

et on notera $L^{1}$ l'espace normé ainsi obtenu. Il est bien connu que $L^{1}$ est complet-autrement dit est un espace de Banach; que $L \subset L^{1}$; et que $L$ (muni de la norme de $\left.L^{1}\right)$ est dense dans $L^{1}$. Plus généralement, $L^{p}(1 \leqq p<+\infty)$ sera l'espace des $\phi(x)$ (sommables sur tout compact) pour lesquelles $|\phi|^{p} \in L^{1}$, normé par

(') A. Weil, l' Intégration dans les groupes topologiques et ses applications (Actualités Scientifiques et Industriélles, no. 869), Paris, 1940. Cité Weil dans la suite. 


$$
\|\phi\|_{p}=\left\{\int|\phi(x)|^{p} d x\right\}^{1 / p}
$$

Ici encore on pourrait obtenir $L^{p}$ en complétant $L$ pour la norme $\|\phi\|_{p}$. De plus, si $f \in L^{p}$, si $f^{\prime} \in L^{p^{\prime}}$ et si

$$
1 / p+1 / p^{\prime}=1
$$

alors $f(x) f^{\prime}(x) \in L^{1}$ et on a

$$
\left|\int f(x) f^{\prime}(x) d x\right| \leqq\left\|f \cdot f^{\prime}\right\|_{1} \leqq\|f\|_{p} \cdot\left\|f^{\prime}\right\|_{p^{\prime}}
$$

$L^{p^{\prime}}$ est du reste, pour $p>1$, le dual de $L^{p}$.

Pour $p=1$, le dual de $L^{1}$ est l'espace $L^{\infty}$ des $\phi(x)$ sommables sur tout compact et bornées sur $G$ (ou "presque partout" sur $G$ ). La norme sera définie dans $D^{\infty}$ par

$$
\|\phi\|_{\infty}=\sup _{f \in L^{1}} \frac{\left|\int f(x) \phi(x) d x\right|}{\|f\|_{1}}=\underset{x \in G}{\operatorname{vraimax}}|\phi(x)|,
$$

en sorte que l'inégalité (I 3) sera remplacée ici par

$$
\left|\int f(x) \phi(x) d x\right| \leqq\|f\|_{1} \cdot\|\phi\|_{\infty} .
$$

On peut maintenant définir comme suit le produit de composition. Associons à toute $f$, sommable sur tout compact, la mesure

$$
d \mu_{p}^{f}(x)=f(x) \frac{d x}{\rho(x)^{1 / p}}
$$

où $p$ est un nombre à priori quelconque (mais $\neq 0$ ). Si $\nu$ est une mesure telle que $\mu_{p}^{f} * \nu$ existe, on aura pour $\phi \in L$, et d'après le Lemme 4:

$$
\begin{aligned}
\int \phi(z) d \mu_{p}^{\prime} * \nu(z) & =\iint \phi(x y) f(x) \frac{d x}{\rho(x)^{1 / p}} d \nu(y) \\
& =\int d \nu(y) \cdot \int \phi(x y) f(x) \frac{d x}{\rho(x)^{1 / p}}=\int d_{\nu}(y) \int \frac{\phi(z) f\left(z y^{-1}\right)}{\rho\left(z y^{-1}\right)^{1 / p}} \frac{d z}{\rho(y)} \\
& =\int d \nu(y) \int \frac{\phi(z) f\left(z y^{-1}\right)}{\rho(z)^{1 / p}} \frac{d z}{\rho(y)^{1 / p^{\prime}}} \quad\left(\frac{1}{p}+\frac{1}{p^{\prime}}=1\right) \\
& =\int \phi(z) \frac{d z}{\rho(z)^{1 / p}} \int f\left(z y^{-1}\right) \frac{d \nu(y)}{\rho(y)^{1 / p^{\prime}}}
\end{aligned}
$$


et par suite

$$
\stackrel{f}{\mu_{p} * \nu}=\stackrel{g}{\mu_{p}}
$$

où $g(x)$ est déterminée par

$$
g(x)=f * \nu(x)=\int f\left(x y^{-1}\right) \frac{d \nu(y)}{\rho(y)^{1 / p^{\prime}}} .
$$

Bien entendu, $g$ dépend de $p$.

Par contre, si $\nu$ est elle-même de la forme

$$
d \mu_{p}^{f^{\prime}}(x)=d \nu(x)=f^{\prime}(x) \frac{d x}{\rho(x)^{1 / p}},
$$

il vient

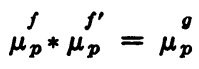

où

$$
g(x)=f * f^{\prime}(x)=\int f\left(x y^{-1}\right) f^{\prime}(y) \frac{d y}{\rho(y)}
$$

c'est à dire

$$
\begin{aligned}
f * f^{\prime}(x) & =\int f(x y) f^{\prime}\left(y^{-1}\right) d y \\
& =\int f(y) f^{\prime}\left(y^{-1} x\right) d y
\end{aligned}
$$

et cette fois la valeur attribuée à $p$ n'intervient pas. Bien entendu, $f * f^{\prime}$, quand elle existe, n'est déterminée en général qu'à un ensemble de mesure nulle près.

Il nous reste à éxaminer les principaux cas où (I 4) et (I 5) ont un sens.

Il est clair que (I 4) a toujours un sense si $f \in L$, et représente une fonction continue-et même une fonction de $L$ si $\nu$ est portée par un compact. Supposons maintenant

$$
\|\nu\|_{1}<+\infty \text {, et } p=2, \quad \text { d'où } p^{\prime}=2 \text {, }
$$

et

$$
f * \nu(x)=\int f\left(x y^{-1}\right) \frac{d \nu(y)}{(\rho(y))^{1 / 2}}
$$

Alors si $\phi \in L$ on aura 


$$
\begin{aligned}
\left|\int \phi(x) f * \nu(x) d x\right| & =\left|\iint \frac{\phi(x) f\left(x y^{-1}\right)}{(\rho(y))^{1 / 2}} d x d \nu(y)\right| \\
& =\left|\int d \nu(y) \int \phi(x) \frac{f\left(x y^{-1}\right)}{(\rho(y))^{1 / 2}} d x\right| \\
& \leqq\|\nu\|_{1} \cdot\|\phi\|_{2} \cdot\left[\int\left|\frac{f\left(x y^{-1}\right)}{(\rho(y))^{1 / 2}}\right|^{2} d y\right]^{1 / 2} \\
& =\|\nu\|_{1} \cdot\|\phi\|_{2} \cdot\left\{\int|f(x)|^{2} d x\right\}^{1 / 2}=\|\nu\|_{1} \cdot\|\phi\|_{2} \cdot\left\|_{f}\right\|_{2}
\end{aligned}
$$

d'où le

LEMME 6(10). Si $f \in L^{2}$ et si $\nu$ est de norme finie, la fonction

$$
f * \nu(x)=\int f\left(x y^{-1}\right) \frac{d \nu(y)}{(\rho(y))^{1 / 2}}
$$

est dans $L^{2}$ et vérifie

$$
\|f * \nu\|_{2} \leqq\|\nu\|_{1} \cdot\|f\|_{2}
$$

Ce lemme prouve que l'opérateur $f \rightarrow f * \nu$ est borné dans l'espace $L^{2}$; on étudiera plus loin cette question (chap. IV). Considérons maintenant (I 5). Si $f, f^{\prime} \in L$, évidemment $f * f^{\prime} \in L$. De plus, si $\phi \in L$ on a

$$
\begin{aligned}
\left|\int \phi(x) f * f^{\prime}(x) d x\right| & =\left|\iint f(x y) f^{\prime}\left(y^{-1}\right) \phi(x) d x d y\right| \\
& =\left|\int f(y) d y \int f^{\prime}\left(y^{-1} x\right) \phi(x) d x\right| \leqq\|f\|_{1} \cdot\left\|f^{\prime}\right\|_{p} \cdot\|\phi\|_{p^{\prime}}
\end{aligned}
$$

d'où (Weil, p. 49) le

Lemme 7. $f * f^{\prime}$ est définie pour $f \in L^{1}, f^{\prime} \in L^{p}$, appartient d $L^{p}$, et vérifie

$$
\left\|f * f^{\prime}\right\|_{p} \leqq\|f\|_{1} \cdot\left\|f^{\prime}\right\|_{p}
$$

Ce resultat est valable pour $1 \leqq p \leqq+\infty$.

4. Regularisation des mesures et des fonctions. Désignons par $\epsilon$ la mesure formée d'une "masse +1 en $e$," et donnée par

$$
\int f(x) d \epsilon(x)=f(e)
$$

Soit $V$ un voisinage compact de $e$, et $f$ une fonction de $L_{0}^{+}$, nulle en dehors de

(10) C'est à H. Cartan que je dois cette proposition, ainsi que l'idée d'introduire (I 16). 
$V$, telle que $\int f(x) d x=1$. Alors quand $V$ converge vers $e$, la mesure $f(x) d x$ converge étroitement vers $\epsilon$. Soit en effet $\phi \in L$; choisissons $\alpha>0$, puis $V$ tel que

$$
x \in V \rightarrow|\phi(x)-\phi(e)|<\alpha
$$

( $\phi$ est continue en $e$ ). Alors on a

$$
\left|\int f(x) \phi(x) d x-\int \phi(x) d \epsilon(x)\right| \leqq \int|\phi(x)-\phi(e)| f(x) d x \leqq \alpha
$$

ce qui prouve la proposition puisque: $f(x) d x$ converge vaguement-et reste portée par un compact fixe.

Dans l'ensemble des mesures positives de normes $\leqq 1$, nous désignerons par $\Phi$ le filtre dont une base est constituée en associant, à chaque voisinage compact $V$ de $e$, les mesures $f(x) d x$ où $f \in L_{0}^{+}$est nulle en dehors de $V$ et vérifie $\int f(x) d x=1$. Ce qui précède prouve que $\Phi$ converge étroitement vers la mesure $\epsilon$.

LEMME 8. Si $\phi(x)$ est continue sur $G$, on a

$$
\phi(x)=\lim _{\Phi} f * \phi(x)
$$

uniformément sur tout compact-et uniformément sur $G$ si $\phi(x)$ est uniformément continue.

Il suffit pour le voir d'écrire

$$
\begin{aligned}
|f * \phi(x)-\phi(x)| & =\left|\int\left[\phi\left(y^{-1} x\right)-\phi(x)\right] f(y) d y\right| \\
& \leqq \int\left|\phi\left(y^{-1} x\right)-\phi(x)\right| f(y) d y
\end{aligned}
$$

et d'observer que $\phi$ est uniformément continue sur tout compact. Par ailleurs, le lemme $5 c$ entraine le

LeMme 9. Si $f(x) d x$ et $g(x) d x$ convergent vers $\epsilon$ suivant le filtre $\Phi$, il en est de méme de $f * g(x) d x$.

Si maintenant $\mu$ est une mesure de Radon, ses "régularisées" seront ses produits de composition avec les $f(x) d x$. L'intérêt de cette notion est que la régularisation fait passer de $\mu$ à une mesure définie par la densité continue

$$
g(x)=\int f\left(x y^{-1}\right) \frac{d \mu(y)}{\rho(y)} .
$$

Lemme 10. Quand $f(x) d x$ converge suivant $\Phi$, la régularisée de $\mu$ par $f$ converge vaguement vers $\mu$. 
En effet, si $\phi \in L$, on a

$$
\begin{aligned}
\int \phi(x) f * \mu(x) d x & =\iint \frac{\phi(x) f\left(x y^{-1}\right)}{\rho(y)} d \mu(y) d x \\
& =\iint \phi(x y) f(x) d x \cdot d \mu(y)=\int d \mu(y) \int \phi(x y) f(x) d x
\end{aligned}
$$

et comme un raisonnement analogue à celui qui a conduit au Lemme 8 prouve que

$$
\phi(y)=\lim _{\boldsymbol{\Phi}} \int \phi(x y) f(x) d x
$$

uniformément sur tout compact, la proposition est démontrée.

Il en résulte que, si $f * \mu(x) \equiv 0$ pour des $f \in L_{0}^{+}$qui s'annulent en dehors de voisinages arbitrairement petits de $e$, la mesure $\mu$ est identiquement nulle.

\section{CHAPITRE I}

\section{FONCTIONS DE TYPE POSITIF ET REPRESENTATIONS UNITAIRES CONTINUES}

\section{A. Proprietes generales des Representations unitaires continues}

5. Définitions. Soient $G$ un groupe topologique localement compact, et ऊC un espace de Hilbert, où l'on désignera par $(X, Y)$ et $|X|$ le produit scalaire et la norme $\left({ }^{11}\right)$.

(a) Par définition une représentation unitaire continue de $G$ dans $\mathfrak{H}$ est une fonction qui associe, à chaque $x \in G$, un opérateur unitaire $U_{x}$ dans $\mathfrak{H}$, de façon que:

(1) $U_{x^{-1} y}=U_{x}^{-1} \circ U_{y}$ quels que soient $x, y \in G$;

(2) Quels que soient $X, Y \in \mathfrak{H}$, la fonction

$$
\left(U_{x} X, Y\right)
$$

soit continue $\operatorname{sur} G$.

Ce n'est donc pas autre chose qu'une représentation (au sens général du terme) de $G$ dans le groupe des opérateurs unitaires de $\mathfrak{T}$ (groupe muni de la topologie faible ou, ce qui revient au même, de la topologie forte $\left.\left({ }^{12}\right)\right)$. Il est essentiel d'observer que la condition (2) est remplie d'clle-même dès qu'elle

(11) Les notations et la terminologie adoptées sont celles de B. von Sz. Nagy, Spektraldarstellung linearer Transformationen des Hilbertschen Raumes (Ergebnisse der Mathematik, t. 5, H.5, Berlin, 1942).

${ }^{(12)} \mathrm{Si}$ un opérateur unitaire variable $U$ converge faiblement vers un opérateur unitaire $U_{0}$, pour chaque $X, U X$ converge faiblement vers $U_{0} X$ (par définition); mais comme au surplus $|U X|=|X| \rightarrow\left|U_{0} X\right|=|X|$, on peut affirmer que $U X$ converge fortement vers $U_{0} X$ : donc (par definition de la topologie forte) $U$ converge fortement vers $U_{0}$. 
l'est pour $X=Y$; ceci d'après l'identité bien connue-et fondamentale pour la suite-qui s'écrit

$$
\begin{aligned}
4\left(U_{x} X, Y\right) \equiv & \left(U_{x}(X+Y), X+Y\right)-\left(U_{x}(X-Y), X-Y\right) \\
& +i\left(U_{x}(X+i Y), X+i Y\right)-i\left(U_{x}(X-i Y), X-i Y\right)
\end{aligned}
$$

On dira qu'une partie $A \subset \mathcal{H}$ est invariante (sous entendu: pour la représentation $U_{x}$ ) si $X \in A$ entraine $U_{x} X \in A$ quel que soit $x \in G$. Il est clair qu'avec $A$ sont également invariantes: les adhérences faible et forte de $A$.dans $\mathcal{B}$, l'enveloppe convexe de $A$, la variété linéaire soustendue par $A$, la variété linéaire fermée soustendue par $A$.

Lemme 11. Si une variêté linéaire fermée $V$ est invariante, il en est de méme de la variêté complémentaire $\left.{ }^{13}\right) \operatorname{Hc} \theta V$. De plus l'invariance de $V$ équivaut d

$$
U_{x} \circ P_{V}=P_{V} \circ U_{x} \quad \text { quel que soit } x \in G \text {. }
$$

(b) Conformément d la terminologie de $N$. Bourbaki, relative aux "structures," nous appellerons structure unitaire l'ensemble $\left\{\mathcal{H C}, U_{x}\right\}$ d'un espace de Hilbert $\mathfrak{H C}$ et d'une représentation unitaire continue $U_{x}$ de $G$ dans $\mathfrak{H}$.

On dira que deux $\left({ }^{14}\right)$ structures unitaire $\left\{\mathfrak{F}, U_{x}\right\}$ et $\left\{\mathcal{H C}^{\prime}, U_{x}^{\prime}\right\}$ sont isomorphes s'il existe un isomorphisme $\phi$ de $\mathfrak{F C}^{\mathrm{C}}$ sur $\mathfrak{F C}^{\prime}$ tel qu'on ait

$$
\phi \circ U_{x}=U_{x}^{\prime} \circ \phi \quad \text { quel que soit } x \in G \text {. }
$$

On dira que la structure unitaire $\left\{\mathcal{F}^{\prime}, U_{x}^{\prime}\right\}$ est contenue dans la structure unitaire $\left\{\mathfrak{F}, U_{x}\right\}$ si la première est isomorphe à $\left\{\mathcal{F}^{\prime \prime}, U_{x}^{\prime \prime}\right\}$, où $\mathfrak{F}^{\prime \prime}$ est un sous-espace fermé de $\Im$ C, invariant par les $U_{x}$, et où $U_{x}^{\prime \prime}$ est la restriction de $U_{x}$ à $\mathfrak{H}^{\prime \prime}$.

Etant données plusieurs structures unitaires $\left\{\mathfrak{C}^{1}, U_{x}^{1}\right\}, \cdots,\left\{\mathfrak{C}^{n}, U_{x}^{n}\right\}$, la formule

$$
U_{x}\left[X^{1}, \cdots, X^{n}\right]=\left[U_{x}^{1} X^{1}, \cdots, U_{x}^{n} X^{n}\right]
$$

définit évidemment une reprěsentation unitaire continue $U_{x}$ de $G$ dans l'espace produit $\left({ }^{15}\right)$

$$
\mathscr{H C}=\mathscr{H C}^{1} \times \cdots \times \mathscr{H C}^{n} .
$$

La structure unitaire ainsi obtenue sera notée

(18) C'est l'ensemble des $X \in \mathcal{H C}$ tels que l'on ait $(X, Y)=0$ pour tout $Y \in V$. La notation $P_{V}$ désigne l'opérateur de projection sur $V$.

(14) Ici comme dans toute la suite, les diverses structures unitaires envisagées sont relatives au même groupe de base $G$.

(15) $\mathcal{H C}^{1} \times \cdots \times \mathcal{H}^{n}$ est l'ensemble des systèmes $\left[X^{1}, \cdots, X^{n}\right]\left(X^{i} \in \mathcal{H C}^{i}\right)$ où l'on a posé $\lambda\left[X^{1}, \cdots, X^{n}\right]=\left[\lambda X^{1}, \cdots, \lambda X^{n}\right] ;\left[X^{1}, \cdots, X^{n}\right]+\left[Y^{1}, \cdots, Y^{n}\right]=\left[X^{1}+Y^{1}, \cdots, X^{n}+Y^{n}\right]$; $\left(\left[X^{1}, \cdots, X^{n}\right],\left[Y^{1}, \cdots, Y^{n}\right]\right)=\left(X^{1}, Y^{1}\right)+\cdots+\left(X^{n}, Y^{n}\right)$. 


$$
\left\{\mathfrak{F C}, U_{x}\right\}=\left\{\mathcal{F C}^{1}, U_{x}^{1}\right\} \times \cdots \times\left\{\mathfrak{F C}^{n}, U_{x}^{n}\right\} ;
$$

il est évident qu'elle "contient" chacune des structures données.

Inversement, soient $\left\{\mathfrak{F}, U_{x}\right\}$ une structure unitaire, et

$$
\mathfrak{C}=\mathfrak{H C}^{1} \oplus \cdots \oplus \mathfrak{H}^{n}
$$

une décomposition de $\mathfrak{H C}$ en sous-espaces invariants fermés deux à deux orthogonaux. Si on note $U_{x}^{d}$ la restriction de $U_{x}$ à $\mathcal{C}^{i}$, on aura évidemment isomorphisme entre

$$
\left\{\mathfrak{F}, U_{x}\right\} \text { et }\left\{\mathfrak{H}^{1}, U_{x}^{1}\right\} \times \cdots \times\left\{\mathfrak{F}^{n}, U_{x}^{n}\right\} .
$$

Une structure unitaire sera dite irréductible si elle ne "contient" pas d'autre structure qu'elle même. Le fait suivant est trivial:

Lemme 12. Pour que $\left\{\mathfrak{H} ; U_{x}\right\}$ soit irréductible, il faut et il suffit que, pour tout élément $X \neq 0$ de $\mathfrak{H}$, la plus petite variêté linéaire fermée de $\mathfrak{H C}$ contenant les $U_{x} X$ soit $\mathfrak{H C}$ lui-même.

On dira que deux structures unitaires sont disjointes s'il n'existe aucune structure unitaire qui soit à la fois contenue dans les deux premières. On peut considérer comme une généralisation du classique lemme de Schur le suivant

Lemme 13. Deux structures unitaires irréductibles quelconques sont ou bien isomorphes ou bien disjointes.

(c) Une structure unitaire simple sur $G$ est l'ensemble $\left\{\mathfrak{x}, U_{x}, X\right\}$ d'une structure unitaire $\left\{\Re, U_{x}\right\}$ et d'un élément distingué $X \in \mathfrak{H}$ tel que la plus variété linéaire fermée contenant les $U_{x} X$ soit $\mathfrak{H}$ tout entier (on dira pour abréger que $X$ "engendre" $\mathfrak{H})$. $\left\{\mathfrak{F}, U_{x}, X\right\}$ sera isomorphe à $\left\{\mathfrak{C C}^{\prime}, U_{x}^{\prime}, X^{\prime}\right\}$ s'il existe un isomorphisme, $\phi$, de $\mathcal{H C}^{\prime}$ sur $\mathcal{H C}^{\prime}$ tel que l'on ait $U_{x}^{\prime} \circ \phi=\phi \circ U_{x}$ et

$$
X^{\prime}=\phi(X) \text {. }
$$

LEMme 14. Soient $\left\{\mathcal{H}, U_{x}\right\}$ une structure unitaire, et $\mathcal{H}^{\prime}$ un sous-espace vectoriel fermé invariant de ${ }^{C}$. Si $X$ engendre ${ }^{C}$, la projection orthogonale $X^{\prime} d e$ $X$ sur $\mathcal{S C}^{\prime}$ engendre $\mathrm{JC}^{\prime}$.

Démonstration. Soit $P_{\mathfrak{K}}$, l'opérateur de projection sur $\mathfrak{K}^{\prime}$ : d'après le Lemme 11, on aura

$$
P_{\mathfrak{H C}} \circ U_{x}=U_{x} \circ P_{\mathfrak{H C}}
$$

Si alors $Y^{\prime} \in \mathcal{H C}^{\prime}$, on peut faire

$$
\left|\sum \alpha_{i} \cdot U_{x_{i}} X-Y^{\prime}\right| \leqq \epsilon
$$

( $\epsilon>0$ arbitraire) d'où, en appliquant $P_{\mathcal{J C}}$, et tenant compte de la permutabilité à $U_{x}$, l'inégalité 


$$
\left|\sum_{i} \alpha_{i} \cdot U_{x_{i}} X^{\prime}-Y\right| \leqq \epsilon
$$

qui prove le lemme.

Le Lémme 14 justifie la définition suivante: $\left\{\mathcal{C}^{\prime}, U_{x}^{\prime}, X^{\prime}\right\}$ sera contenue dans $\left\{\mathfrak{H}, U_{x}, X\right\}$ si la première structure est isomorphe à $\left\{\mathfrak{C}^{\prime \prime}, U_{x}^{\prime \prime}, X^{\prime \prime}\right\}$ où $\mathcal{H}^{\prime \prime}$ est un sous-espace vectoriel fermé invariant de $\mathcal{H}, U_{x}^{\prime \prime}$ la restriction de $U_{x}$ à $\mathcal{H}^{\prime \prime}$, et $X^{\prime \prime}$ la projection orthogonale de $X$ sur $\mathcal{H}^{\prime \prime}$.

La nécessité de considérer les structures unitaires simples provient du Lemme 12.

6. Operateurs de Radon. Soient $\left\{\mathscr{C}, U_{x}\right\}$ une structure unitaire, et $\mu$ une mesure de Radon (en général complexe) sur $G$, et de masse totale finie. Alors, quels que soient $X, Y \in \Re$, l'expression

$$
\int\left(U_{x} X, Y\right) d \mu(x)
$$

a un sens, et constitue évidemment une fonctionnelle bilinéaire bornée de $X, Y$; donc on peut écrire $\left.{ }^{16}\right)$

$$
\int\left(U_{x} X, Y\right) d \mu(x)=\left(T_{\mu} X, Y\right)
$$

où $T_{\mu}$ est un opérateur linéaire borné, dont la norme est majorke par la norme de $\mu$.

Si $\mu, \nu$ sont deux telles mesures, on aura

$$
\begin{aligned}
\left(T_{\nu} T_{\mu} X, Y\right) & =\int\left(U_{x} T_{\mu} X, Y\right) d \nu(x)=\int\left(T_{\mu} X, U_{x^{-1}} Y\right) d \nu(x) \\
& =\int d \nu(x) \int\left(U_{y} X, U_{x^{-1}} Y\right) d \mu(y) \\
& =\iint\left(U_{x y} X, Y\right) d \nu(x) d \mu(y)
\end{aligned}
$$

ce qui prouve que

$$
T_{\nu} \circ T_{\mu}=T_{\nu * \mu}
$$

D'autre part, l'adjoint $T_{\mu}^{*}$ de $T_{\mu}$ est donné par

$$
\left(T_{\mu}^{*} X, Y\right)=\overline{\left(T_{\mu} Y, X\right)}=\int\left(U_{x} X, Y\right) d \overline{\mu\left(x^{-1}\right)}
$$

(16) On pourrait aussi bien poser directement $T_{\mu}=\int U_{x} \cdot d_{\mu}(x)$ où l' $\int$ est à interpréter comme intégrale d'une fonction à valeurs dans l'espace des opérateurs, muni de la topologie forte. Cf. par exemple les travaux de $\mathrm{S}$. Bochner sur l'intégration des fonctions à valeurs dans un espace de Banach. 
et par suite on a

$$
T_{\mu}^{*}=T_{\mu} \sim
$$

où $\mu^{\sim}$ est la mesure définie symboliquement par

$$
d \mu \sim(x)=d \overline{\mu\left(x^{-1}\right)} ;
$$

de (I 9) et (I 10) résulte que $T_{\mu_{*} \mu} \sim$ est hermitien positif-en d'autres termes, on a l'inégalité fondamentale

$$
\iint\left(U_{x^{-1} y} X, X\right) d \mu(x) d \overline{\mu(y)} \geqq 0
$$

valable quels que soient $X \in \mathcal{H C}^{\mathrm{C}}$ et la mesure bornée $\mu$. Il est clair par ailleurs que, si $\epsilon_{x}$ désigne la "masse 1 en $x$," on a

$$
T_{a x}=U_{x} .
$$

D'autre part, si $X \in \mathfrak{F C}$ engendre $\mathfrak{H C}$, les $T_{\mu} X$ correspondant à toutes les $\mu$ bornées sont denses dans $\Re$ : car ils comprennent déjà les

$$
\sum \alpha_{i} \cdot U_{x_{i}} X
$$

correspondant aux mesures discretes (masse $\alpha_{i}$ en $x_{i}$ ).

Supposons maintenant que la mesure $\mu$ converge étroitement vers $\mu_{0}$. On va prouver la

PRoposition 1. Si $\mu$ de norme uniformément bornée converge êtroitement vers $\mu_{0}$, l'opérateur $T_{\mu}$ converge fortement dans $\operatorname{TC}$ vers $T_{\mu_{0}}$.

Démonstration. Il faut prouver que, pour chaque $X, T_{\mu} X$ converge fortement vers $T_{\mu_{0}} X$. Tout d'abord, l'inégalité

$$
\left|T_{\mu} X\right| \leqq\|\mu\|_{1}:|X|
$$

prouve que les $T_{\mu} X$ restent dans une boule de 3 .

Ensuite, pour tout $Y$,

$$
\left(T_{\mu} X, Y\right)=\int\left(U_{x} X, Y\right) d \mu(x)
$$

tend vers $\left(T_{\mu_{0}} X, Y\right)$ puisque le convergence de $\int \phi d \mu$ a lieu pour toute $\phi$ continue bornée (Lemme 2 ).

Donc $T_{\mu} X$ converge faiblement vers $T_{\mu_{0}} X$. Par conséquent, pour chaque $Y \in \mathcal{H}$, la fonction

$$
\phi(x)=\left(T_{\mu} X, U_{x} Y\right)
$$

converge vers

$$
\phi_{0}(x)=\left(T_{\mu_{0}} X, U_{x} Y\right)
$$


uniformément sur tout compact de $G$ (car $U_{x}$ étant faiblement et donc fortement continue, l'ensemble des $U_{x} Y$ où $x$ varie dans un compact de $G$ est une partie fortement compacte de $\mathfrak{H}$-la proposition résulte alors d'un résultat bien connu qu'on trouvera dans Gelfand $\left({ }^{17}\right)$ ) en restant uniformément bornée $\operatorname{sur} G$.

Alors, comme $\mu$ converge étroitement vers $\mu_{0}$, on voit facilement que $\int \dot{\phi} d \mu$ tends vers $\int \phi_{0} d \mu_{0}$ : ce qui signifie que

$$
\left|T_{\mu} X\right|^{2} \rightarrow\left|T_{\mu_{0}} X\right|^{2}
$$

et finalement $T_{\mu} X$ tend fortement vers $T_{\mu_{0}} X$.

Remarque. L'essentiel de cette proposition est dû à Gelfand et Raïkov.

B. Proprietes Generales Des fonctions DE TyPe POSITIF

7. Fonction caractéristique d'une structure unitaire simple. $\left\{\mathfrak{K}, U_{x}, X\right\}$ étant une structure unitaire simple, on notera fonction caractéristique de cette structure la fonction (continue)

$$
\phi(x)=\left(X, U_{x} X\right) .
$$

L'inégalite (I 11) prouve que $\phi$ vérifie

$$
\iint \phi\left(x^{-1} y\right) d \mu(x) \cdot d \overline{\mu(y)} \geqq 0
$$

pour toute mesure $\mu$ de norme finie sur $G$. En particulier, si $f \in L^{1}$, on aura

$$
\iint \phi\left(x^{-1} y\right) f(x) \overline{f(y)} d x d y \geqq 0 ;
$$

et si $\mu$ consiste en masses discrètes $\alpha_{i}$ placées aux points $x_{i} \in G$, on aura

$$
\sum \phi\left(x_{i}^{-1} x_{j}\right) \alpha_{i} \bar{\alpha}_{j} \geqq 0 .
$$

Comme toute $\mu$ de norme finie est limite étroite de mesures de la forme $f(x) d x$, ou de mesures discrètes, les propriétés (I 12), (I 12') et (I 12') sont équivalentes pour une $\phi$ continue et bornée sur $G$; on la verra du reste autrement plus loin. Notre but est pour l'instant de prouver le

Theoreme 1. Toute structure unitaire simple est déterminee, à un isomorphisme près, par la donnée de sa fonction caractéristique.

Démonstration. (1) Désignons par $C^{\infty}$ l'ensemble des fonctions continues

(17) Le lemme de Gelfand-dont on se servira plusieurs fois dans la suite-est le suivant: soit $A$ une partie fortement compacte d'un espace de Banach $E$; soit $\mu(x)$ une fonctionnelle lineaire continue définie sur $E$, qui converge faiblement vers une $\mu_{0}(x)$ en restant de norme uniformément bornée; alors $\mu(x) \rightarrow \mu_{0}(x)$ uniformément pour $x \in A$. Cf. Rec. Math. (Mat. Sbornik) N.S. (1938) (Abstrakte funktionen und lineare Operatoren). 
de $L^{\infty}$ : nous allons prouver tout d'abord qu'il existe une application linéaire, biunivoque et continue $\lambda$ de $\mathfrak{H C}$ sur un sous-espace $H_{\phi}$ de $C^{\infty}$, application qui amène l'élément distingué $X \in \mathcal{H C}$ sur la fonction $\phi \in C^{\infty}$, et qui transforme

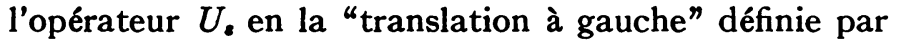

$$
f(x) \rightarrow f_{s}(x)=f\left(s^{-1} x\right) .
$$

Pour cela, associons à chaque $Y \in \mathfrak{H C}$ la fonction $\psi=\lambda(Y)$ définie par

$$
\psi(x)=\left(Y, U_{x} X\right) .
$$

Il est clair que $\lambda$ est une application linéaire de $\mathfrak{H C}$ dans $C^{\infty}$; soit $H_{\phi}$ l'image de ${ }^{3} \operatorname{par} \lambda$. $\lambda$ est biunivoque parce que $\lambda(Y)=0$ équivaut à

$$
\left(Y, U_{x} X\right)=0
$$

pour tout $x \in G$

et donc, puisque les $U_{x} X$ soustendent $\mathfrak{x}$, à $Y=0$. Enfin $\lambda$ est continue parce que

$$
\|\lambda(Y)\|_{\infty}=\sup _{x \in G}\left|\left(Y, U_{x} X\right)\right| \leqq|Y| \cdot|X|=K \cdot|Y|
$$

où $K$ ne dépend pas de $Y$.

On a d'autre part, si $\psi=\lambda(X)$ :

$$
\psi(x)=\left(X, U_{x} X\right)=\phi(x)
$$

donc $\phi=\lambda(X)$. Enfin si $\psi=\lambda(Y)$, à $U_{\triangleleft} Y$ correspond la fonction

$$
\left(U_{s} Y, U_{x} X\right)=\left(Y, U_{s^{-1}} X\right)=\psi\left(s^{-1} x\right)=\psi_{\diamond}(x)
$$

d'où finalement $\psi_{s}=\lambda\left(U_{s} Y\right)$ comme annoncé. Il est clair que, les $U_{s} X$ soustendant $\Re$, toute $\psi \in H_{\phi}$ est limite uniforme de combinaisons linéaires de translatées à gauche de $\phi(x)$.

$$
\text { Soient } \begin{aligned}
\psi & =\lambda(Y), & \theta & =\lambda(Z) \quad \text { où } \\
Y & =\sum_{i} \alpha_{i} \cdot U_{s_{i}} X, & Z & =\sum_{i} \beta_{i} \cdot U_{t_{j}} X ;
\end{aligned}
$$

on a donc

$$
\psi=\sum_{i} \alpha_{i} \phi_{s_{i}}, \quad \theta=\sum_{i} \beta_{j} \phi_{t_{j}} .
$$

D'autre part, on a

$$
\begin{aligned}
(Y, Z) & =\left(\sum \alpha_{i} U_{s_{i}} X, \sum \beta_{i} \cdot U_{t_{j}} X\right) \\
& =\sum \alpha_{i} \bar{\beta}_{j}\left(U_{s_{i}} X, U_{t_{i}} X\right)=\sum_{i_{i} j} \alpha_{i} \bar{\beta}_{j} \phi\left(s_{i}^{-1} t_{j}\right) ;
\end{aligned}
$$

par suite, le produit scalaire $(Y, Z)$, pour des éléments de la forme (1):

(1) s'exprime uniquement à l'aide de la fonction $\phi$; 
(2) dépend uniquement des fonctions $\psi, \theta \in H_{\phi}$ associées à $Y, Z$. Ces remarques, et le fait que les $Y$ de la forme (1) sont denses dans $\mathfrak{H C}$ suffisent à prouver le théorème $I$.

Le théorème 1 conduit naturellement à la question suivante: quelles sont les fonctions $\phi(x)$ qu'on peut obtenir à partir d'une structure unitaire simple? La réponse est fournie par le

TheOREME 2. Une condition nécessaire et suffisante pour qu'une $\phi(x)$ soit la fonction caractéristique d'une structure unitaire simple est qu'elle soit continue et vérifie

$$
\sum_{i, j} \alpha_{i} \bar{\alpha}_{j} \phi\left(s_{i}^{-1} s_{j}\right) \geqq 0
$$

quels que soient les nombres complexes $\alpha_{i}$ et les éléments $s_{i} \in G$.

Démonstration. (a) (I $\left.12^{\prime \prime}\right)$ entraine que, quels que soient les $s_{j} \in G$, la forme quadratique de coefficients $\phi\left(s_{i}^{-1} s_{j}\right)$ est définie positive: on en déduit

$$
\phi(e) \geqq 0, \quad|\phi(x)| \leqq \phi(e), \quad \phi\left(x^{-1}\right)=\overline{\phi(x)} ;
$$

donc, en particulier, $\phi \in C^{\infty}$.

(b) Soit $V_{\phi}$ l'ensemble des combinaisons linéaires de translatées à gauche de $\phi . \mathrm{Si}$

$$
\psi(x)=\sum \alpha_{i} \cdot \phi_{s_{i}}(x), \quad \theta(x)=\sum \beta_{i} \cdot \phi_{t_{j}}(x)
$$

sont deux éléments de $V_{\phi}$, posons

$$
\begin{aligned}
(\psi, \theta)_{\phi} & =\sum \alpha_{i} \bar{\beta}_{j} \phi\left(s_{i}^{-1} t_{j}\right)=\sum \overline{\beta_{j}} \psi\left(t_{i}\right) \\
& =\sum \alpha_{i} \overline{\beta_{j} \phi\left(t_{j}^{-1}\right.} \overline{\left.s_{i}\right)}=\sum \alpha_{i} \overline{\theta\left(s_{i}\right) ;}
\end{aligned}
$$

(3) prouve que, pour $\theta$ donné, $(\psi, \theta)_{\phi}$ ne dépend pas de la représentation particulière (2) de $\psi$; de même, $\left(3^{\prime}\right)$ montre que $(\psi, \theta)_{\phi}$ ne dépend que de $\theta$ : finalement, $(\psi, \theta)_{\phi}$ est entièrement déterminè par les éléments $\psi$ et $\theta$ de $V_{\phi} .(\psi, \theta)_{\phi}$ possède évidemment toutes les propriétés de linéarité habituelles d'une produit scalaire; on a de plus

$$
(\psi, \theta)_{\phi}=\overline{(\theta, \psi)}_{\phi} \text { d'après } \quad \phi\left(x^{-1}\right)=\overline{\phi(x)}
$$

et

$$
(\psi, \psi)_{\phi} \geqq 0 \quad \text { d'après } \quad\left(\mathrm{I} 12^{\prime \prime}\right) \text {. }
$$

De plus

$$
\left(\psi_{s}, \theta_{s}\right)_{\phi}=\sum \alpha_{i} \bar{\beta}_{j} \phi\left(s_{i}^{-1} s^{-1} \cdot s t_{j}\right)=(\psi, \theta)_{\phi}
$$

et (3) prouve que

$$
\psi(x)=\left(\psi, \phi_{x}\right)_{\phi}
$$


Pour voir que $(\psi, \theta)_{\phi}$ est un véritable produit scalaire, il faut prouver que $(\psi, \psi)_{\phi}=0$ entraine $\psi=0$. Or l'inégalité de Cauchy-Schwarz:

$$
\left|(\psi, \theta)_{\phi}\right|^{2} \leqq(\psi, \psi)_{\phi} \cdot(\theta, \theta)_{\phi}
$$

montre qu'alors on a $(\psi, \theta)_{\phi}=0$ quel que soit $\theta \in V_{\phi}$, et donc en particulier

$$
\psi(x)=\left(\psi, \phi_{x}\right)_{\phi}=0 \text { pour tout } x \in G ; \text { d'où } \psi=0 .
$$

(c) $(\psi, \theta)_{\phi}$ étant, sur $V_{\phi}$, un véritable produit scalaire, on peut introduire le complété de $V_{\phi}$ pour la structure hilbertienne correspondante $\left({ }^{18}\right)$, soit $\mathcal{F}_{\phi}$. Mais (4) et (5) montrent que

$$
\|\psi\|_{\infty} \leqq(\phi(e))^{1 / 2} \cdot\left((\psi, \psi)_{\phi}\right)^{1 / 2}
$$

en sorte que, sur $V_{\phi}$, toute "suite de Cauchy" relative à la norme $\left((\psi, \psi)_{\phi}\right)^{1 / 2}$ l'est aussi pour la norme $\|\psi\|_{\infty}$ : il en résulte que tout élément "idéal" $X \in \mathcal{J C}_{\phi}$ peut être réalisé dans $C^{\infty}$ par une fonction $\psi$, qui n'est autre, naturellement, que la fonction

$$
\psi(x)=\left(X, \phi_{x}\right)_{\phi} .
$$

(d) Soit $H_{\phi}$ la réalisation ainsi obtenue de $\mathfrak{K}_{\phi}$ dans $C^{\infty}$. Pour $\psi \in H_{\phi}$, posons

$$
U_{s} \psi=\psi_{s}, \text { d'où } U_{s}^{-1} \psi=\psi_{s}^{-1} .
$$

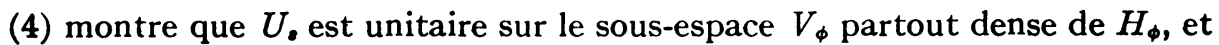
donc dans tout $H_{\phi}$. Que $U$, soit une représentation de $G$ est évident. Enfin, si $\psi, \theta \in V_{\phi}$ on a

$$
\left(U_{s} \psi, \theta\right)_{\phi}=\sum \alpha_{i} \bar{\beta}_{j} \phi\left(s_{i}^{-1} s^{-1} t_{j}\right)
$$

en sorte que, $\phi(s)$ étant continue, $U_{\varepsilon}$ est une fonction faiblement continue de $s$ sur $V_{\phi}$, et donc sur $H_{\phi}$.

Il nous reste à montrer que l'élément $\phi \in H_{\phi}$ "engendre" $H_{\phi}$ : ce qui est trivial, puisque les $\sum \alpha_{i} U_{i_{i}} \phi \in V_{\phi}$ sont denses dans $H_{\phi}$, et le théorème 2 est ainsi complétement démontré.

Remarque. Par la suite, et afin d'éviter toute confusion, on emploiera les notations suivantes:

(1) $\mathfrak{H}_{\phi}$ désignera l'espace de Hilbert abstrait (déterminé à un isomorphisme près) obtenu en complétant $V_{\phi}$ par la structure $(\psi, \theta)_{\phi}$.

(2) $H_{\phi}$ désignera le sous-espace correspondant de $C^{\infty}$, et sera toujours muni de la topologie de $C^{\infty}$ - et non de la structure hilbertienne précédente. Naturellement, $H_{\phi}$ n'est pas nécessairement complet pour cette topologie (c'est à dire: fermé dans $C^{\infty}$ ).

8. Premières propriétés des fonctions continues de type positif. On

(18) B. von Sz. Nagy, ouvrage cité, p. 4. 
appellera désormais, conformément à la terminologie habituelle, fonctions de type positif celles qui vérifient (I 12') : si elles sont continues sur $G$, ce sont les fonctions caractéristiques de structures unitaires simples. Ce fait a des conséquences importantes dont on va donner ici les plus immédiates (on sousentendra toujours le mot continue).

(a) Toute fonction de type positif est bornée sur $G$, et on a

$$
\|\phi\|_{\infty}=\phi(e) \text {. }
$$

(b) Toute fonction de type positif est uniformément continue à gauche et à droite sur $G$. En effet, on peut mettre $\phi$ sous la forme

$$
\phi(x)=\left(X, U_{x} X\right)
$$

d'où

$$
\begin{aligned}
|\phi(x)-\phi(y)|^{2} & \leqq|X|^{2} \cdot\left|U_{x} X-U_{y} Y\right|^{2} \\
& \leqq \phi(e) \cdot\left(U_{x} X-U_{y} Y, U_{x} X-U_{y} Y\right) \\
& =\phi(e) \cdot\left[2(X, X)-2 \Re\left(X, U_{x^{-1} y} X\right)\right]
\end{aligned}
$$

ou enfin

$$
|\phi(x)-\phi(y)|^{2} \leqq 2 \phi(e) \cdot\left[\phi(e)-\Re \phi\left(x^{-1} y\right)\right] .
$$

(Cette inégalité est due, dans le cas général, à Gelfand-Raïkov.) (I 13) prouve évidemment la continuité uniforme $\operatorname{sur} G$, et même plus, à savoir la

Proposition 2. Pour qu'une famille de fonctions de type positif uniformement bornées soit egalement continue en tout point de $G$, il faut et il suffit qu'elle le soit a l'unité de $G$.

(c) L'identité (I 7) prouve sans qu'il soit utile d'insister la

Proposition 3. L'ensemble des combinaisons linéaires de fonctions de type positif est invariant par les translations (à gauche et d droite) de G.

(d) $\mathrm{Si} \mu$ est une mesure de masse totale finie, on a

$$
\left(T_{\mu} \phi, U_{x} T_{\mu} \phi\right)_{\phi}=\iint \phi\left(s^{-1} x t\right) d \mu(s) \overline{d \mu(t)}
$$

et cette fonction est encore de type positif; en particulier, on a, comme déja dit,

$$
\iint \phi\left(s^{-1} t\right) d \mu(s) \overline{d \mu(t)} \geqq 0 .
$$

9. Mesures de type positif. Soit $\phi$ une fonction continue de type positif. On a, pour toute $f \in L$, 


$$
\iint \phi\left(x^{-1} y\right) f(x) \overline{f(y)} d x d y \geqq 0
$$

ou encore

$$
\iint \phi(y) f(x) \overline{f(x y)} d x d y \geqq 0
$$

ou, d'après $\phi\left(y^{-1}\right)=\overline{\phi(y)}$ :

$$
\iint \phi\left(y^{-1}\right) \overline{f(x)} f(x y) d x d y \geqq 0 ;
$$

introduisons

$$
f^{\sim}(x)=\overline{f\left(x^{-1}\right)}
$$

alors (I 12') s'écrit, comme on le vérifie aisement:

$$
f * \phi * f^{\sim}(e) \geqq 0 .
$$

Il est clair que (I 14) a un sens si l'on, remplace $\phi$ par une mesure $\mu$ quelconque; plus précisément on dira qu'une mesure $\mu$ est de type positif sur $G$ si, pour toute $f \in L$, on a

$$
f * \mu * f^{\sim}(e) \geqq 0 .
$$

(Précisons que, comme toujours dans la suite, on associe à toute fonction $f$ la mesure $f(x) d x /(\rho(x))^{1 / 2}$.) On peut expliciter (I 15): on trouve

$$
\iint f\left(x y^{-1}\right) \overline{f(x)} \frac{d \mu(y)}{(\rho(y))^{1 / 2}} d x \geqq 0 .
$$

En particulier, dire que la fonction $\phi(x)$ est de type positif équivaut à dire que la mesure

$$
d \mu^{\phi}(x)=\phi(x) \cdot \frac{d x}{(\rho(x))^{1 / 2}}
$$

est de type positif.

En appliquant (I 15') à la fonction (de $L)$

$$
f(x)=\frac{g\left(x^{-1}\right)}{(\rho(x))^{1 / 2}}
$$

on trouve facilement

$$
\int g * g \sim(x) d \mu(x) \geqq 0
$$


valable pour toute $g \in L$ et toute $\mu$ de type positif. Il est évident que toute mesure de type positif $\mu$ définite une structure unitaire sur $G$ : on l'obtient en introduisant dans $L$ le produit scalaire

$$
(f, g)_{\mu}=f * \mu * g \sim(e)
$$

et en associant $U_{\text {a }}$ à $f_{\text {s. }}$ La seule difficulté qui se présente ici-et qui n'avait pas lieu dans la démonstration du Théorème 2-est que $(f, f)_{\mu}=0$ n'entraine pas nécessairement $f=0$. Mais les $f \in L$ telles que $(f, f)_{\mu}=0$ forment un sousespace $L_{\mu}$ de $L$-évidemment invariant par translation à gauche, et l'on doit considérer $(f, g)_{\mu}$ comme défini, non dans $L$, mais dans l'espace quotient $L / L_{\mu}$. On obtient alors la structure unitaire cherchée $\left\{\mathfrak{C}_{\mu}, U_{x}\right\}$ en complétant $L / L_{\mu}$ comme on l'a fait au théorème 2 pour $\mathfrak{B}_{\phi}$.

La proposition suivante, due pour l'essentiel( $\left.{ }^{(9)}\right)$ à Gelfand et Raïkov, est fondamentale:

Theoreme 3. Si une mesure $\mu$ de type positif vérifie

$$
f * \mu * f^{\sim}(e) \leqq K\left(\int f(x) d x\right)^{2}
$$

pour les $f \in L_{0}^{+}$mulles en dehors d'un voisinage donné de l'unité, elle est de la forme

$$
d \mu(x)=\phi(x) \frac{d x}{(\rho(x))^{1 / 2}}
$$

où $\phi(x)$ est une fonction continue de type positif.

Démonstration. Supposons (a) valable pour les $f \in L_{0}^{+}$qui sont nuulles en dehors de $V$, et considérons, parmi ces $f$, celles qui servent à constituer le filtre $\Phi$ déja considéré ( $\S$ introductif: no. 4 ) : $f \in L_{0}^{+}$avèc

$$
\int f(x) d x=1,
$$

telles que $f(x) d x$ converge vaguement (donc étroitement) vers la masse 1 en $e$. Les image de ces $f$ dans $\mathfrak{K C}_{\mu}$ restent dans une boule de $\mathfrak{K C}_{\mu}$-car (a) s'écrit

$$
(f, f)_{\mu} \leqq K .
$$

D'autre part, quand $f(x) d x$ converge suivant $\Phi$, l'expression

$$
(g, f)_{\mu}=g * \mu * f^{\sim}(e)=\int g * \mu(x) \overline{f(x)} d x
$$

(19) Le résultat de Gelfand-Rarkov concerne le cas où $d \mu(x)=\phi(x) d x /(\rho(x))^{1 / 2}$ avec $\phi \in L^{\infty}$ Notre démonstration, inspirée de la leur, est d'ailleurs plus simple. 
converge 'vers $g * \mu(e)$; comme les $g \in L$ sont denses dans $\mathfrak{K}_{\mu}$, il en résulte que $f$ converge faiblement, dans $\mathfrak{H}_{\mu}$, vers un élément $\epsilon$ tel que $(g, \epsilon)_{\mu}=g * \mu(e)$ pour toute $g \in L$; d'où résulte que

$$
\begin{aligned}
\left(g, U_{x} \epsilon\right)_{\mu} & =\left(U_{x^{-1}} g, \epsilon\right)_{\mu}=g_{x^{-1} * \mu(e)} \\
& =\int g\left(x y^{-1}\right) \frac{d \mu(y)}{(\rho(y))^{1 / 2}}=g * \mu(x) ;
\end{aligned}
$$

et par suite, pour toute $f \in L$, on a

$$
(g, f)_{\mu}=\int g * \mu(x) \overline{f(x)} d x=\int\left(g, U_{x} \epsilon\right)_{\mu} \cdot \overline{f(x)} d x=\left(g, T_{f \epsilon}\right)_{\mu}
$$

où $T_{f}$ est l'opérateur de Radon associé, dans $\mathfrak{H C}_{\mu}$, à la mesure $f(x) d x$.

Supposons alors que $g(x) d x$ converge suivant $\Phi$. Le raisonnement fait plus haut prouve que $g$ converge faiblement vers $\epsilon$ : on a donc

$$
(\epsilon, f)_{\mu}=\left(\epsilon, T_{f} \epsilon\right)_{\mu}
$$

c'est à dire

$$
\overline{f * \mu(e)}=\int\left(\epsilon, U_{x} \epsilon\right)_{\mu} \cdot \overline{f(x)} d x
$$

on enfin en posant $\phi(x)=\left(\epsilon, U_{x} \epsilon\right)_{\mu}$ :

$$
\int \overline{f\left(x^{-1}\right)} \frac{\overline{d \mu(x)}}{(\rho(x))^{1 / 2}}=\int \phi(x) \overline{f(x)} d x=\int \phi\left(x^{-1}\right) \overline{f\left(x^{-1}\right)} \frac{d x}{\rho(x)} .
$$

Cette équation étant valable quel que soit $f \in L$, il en résulte comme annoncé

$$
\frac{\overline{d \mu(x)}}{(\rho(x))^{1 / 2}}=\phi\left(x^{-1}\right) \frac{d x}{\rho(x)}=\overline{\phi(x)} \frac{d x}{\rho(x)}
$$

c'est à dire

$$
d \mu(x)=\phi(x) \frac{d x}{(\rho(x))^{1 / 2}}
$$

où $\phi(x)=\left(\epsilon, U_{x} \epsilon\right)_{\mu}$ est continue et de type positif.

Conséquences $d u$ Théorème 3. Nous noterons $P$ la classe des $\phi(x)$, sommables sur tout compact, telles que la mesure $\phi(x) d x /(\rho(x))^{1 / 2}$ soit de type positif.

On a à ce sujet la

Proposition 4. Toute fonction de $P$ bornée dans un voisinage de l'unité coincide presque partout avec une fonction continue de type positif. 
Car si $|\phi(x)| \leqq K$ presque partout dans $V(e)$, on a pour $f \in L$ nulle en dehors de $W(e)\left(W^{-1} \cdot W \subset V\right)$ :

$$
\begin{aligned}
f * \phi * f^{\sim}(e) & =\iint \phi\left(x^{-1} y\right) f(x) \overline{f(y)} d x d y \\
& \leqq K \cdot\left(\int|f(x)| d x\right)^{2} .
\end{aligned}
$$

En particulier, $P \cap L^{\infty}$ se réduit aux fonctions continues de type positifclasse qu'on notera $P$. (Ceci est le théorème de Gelfand et Raikov.)

Par ailleurs, on peut introduire dans l'ensemble des mesures de type positif une relation d'ordre qu'on notera $\mu \ll \nu$ et qui signifie que $\nu-\mu$ est elle-même de type positif. On a alors de façon évidente $\left({ }^{20}\right)$ la

Proposition 5. Toute mesure $\mu$ de type positif "majoré" par une mesure de la forme

$$
d \nu(x)=\phi(x) \frac{d x}{(\rho(x))^{1 / 2}}
$$

où $\phi(x)$ est continue de type positif, est elle-méme de la forme

$$
d \mu(x)=\psi(x) \frac{d x}{(\rho(x))^{1 / 2}}
$$

où $\psi(x)$ est continue de type positif.

\section{CHAPITRE II}

\section{RELATIONS D'ORDRE-THEOREMES D'EXISTENCE ET D'APPROXIMATION}

\section{A. Theorie DEs Partitions Dans $\boldsymbol{P}$}

10. Partitions et structures unitaires. L'ensemble des fonctions de type positif continues sur $G$ constitue une famille $P$ dans laquelle ont évidemment un sens les opérations $\phi+\psi$ et $\lambda \phi(\lambda \geqq 0)$. On y a introduit à la page précédente la relation d'ordre

$\phi \ll \psi$ équivalente à $\psi-\phi \in \mathbb{P}$.

$\phi \ll \psi$ est aussi le critère de résolubilité, dans $P$, de l'équation à une inconnue $\theta \in \mathcal{P}$

$$
\psi=\phi+\theta \text {. }
$$

Il est trivial que $\phi \ll \psi$ entraine $\lambda \phi \ll \lambda \psi(\lambda \geqq 0)$; et que

(20) En effet, on a pour $f \in L, f_{* \mu * f} \sim(e) \leqq f_{* \nu * f} \sim(e) \leqq \phi(e) \cdot\left(\int|f(x)| d x\right)^{2}$; ce qui ramène au Theorème 3. 


$$
\left.\begin{array}{c}
\phi \ll \psi \\
\phi^{\prime} \ll \psi^{\prime}
\end{array}\right\} \quad \text { entraine } \quad \phi+\phi^{\prime} \ll \psi+\psi^{\prime} .
$$

Enfin, il n'est peut être pas superflu d'expliciter (II-1), qui signifie

$$
\iint \phi\left(x^{-1} y\right) d \mu(x) \overline{d \mu(y)} \leqq \iint \psi\left(x^{-1} y\right) d \mu(x) \overline{d \mu(y)} .
$$

pour toute mesure $\mu$ de masse totale finie.

Le but de ce $\S$ est d'appliquer à l'étude de la relation d'ordre les résultats du chapitre précédent. Le premier problème que nous résoudrons est le suivant: appelons partition d'une $\phi \in \mathcal{P}$ tout ensemble fini de fonctions $\phi_{1}, \cdots, \phi_{n} \in \mathcal{P}$ telles que l'on ait $\phi=\phi_{1}+\cdots+\phi_{n}$; comment peut-on relier les structures unitaires simples associées aux fonctions $\phi, \phi_{1}, \cdots, \phi_{n}$ ?

Pour ne pas alourdir inutilement l'exposé, on supposera $n=2$; mais les raisonnements que l'on fera sur ce cas particulier (le seul qui soit important pratiquement, d'ailleurs) s'étendront d'eux-mêmes au cas général.

Rappelons que, si $\phi \in \mathcal{P}$, on désigne par:

(1) $\left\{\mathcal{H}_{\phi}, U_{x}\right\}$ la structure unitaire "abstraite" associée à $\phi$;

(2) $H_{\phi}$ le sous-espace de $C^{\infty}$ associé à $\phi$.

(Cf. la Remarque, p. 22.)

Ceci étant, on va démontrer le

TheOREME 4. Si $\phi=\phi_{1}+\phi_{2}\left(\phi_{1}, \phi_{2} \in \mathcal{P}\right)$, la structure unitaire $\left\{\mathcal{H}_{\phi}, U_{x}\right\}$ est contenue dans le produit $\left\{\mathfrak{F}_{\phi_{1}}, U_{x}\right\} \times\left\{\mathfrak{F}_{\phi_{2}}, U_{x}\right\}$, et $H_{\phi}$ est, dans $C^{\infty}$, somme des sous-espaces $H_{\phi_{1}}$ et $H_{\phi_{2}}$ (en particulier, $H_{\phi_{1}} \subset H_{\phi}$ ).

Démonstration. (1) Dans le produit direct $\mathfrak{K}_{\phi_{1}} \times \mathfrak{F}_{\phi_{2}}$, les transformés de l'élément $\left[\phi_{1}, \phi_{2}\right]$ de ce produit soustendent une variété linéaire fermée $\mathfrak{H C} \subset \mathcal{H}_{\phi_{1}} \times \mathfrak{H}_{\phi_{2}}$ invariante par les $U_{x}$. La fonction caractéristique de la structure unitaire simple $\left\{\mathcal{H}, U_{x},\left[\phi_{1}, \phi_{2}\right]\right\}$ ainsi obtenue est

$$
\left(\left[\phi_{1}, \phi_{2}\right], U_{x}\left[\phi_{1}, \phi_{2}\right]\right)=\left(\phi_{1}, U_{x} \phi_{1}\right)+\left(\phi_{2}, U_{x} \phi_{2}\right)=\phi_{1}(x)+\phi_{2}(x)=\phi(x):
$$

par suite $\left\{\mathfrak{C}_{\phi}, U_{x}, \phi\right\}$ est isomorphe à cette structure (Théorème 1 ), en sorte que $\left\{\mathfrak{H}_{\phi}, U_{x}\right\}$ est contenue (definition p. 15) dans $\left\{\mathfrak{H}_{\phi_{1}}, U_{x}\right\} \times\left\{\mathfrak{C}_{\phi_{2}}, U_{x}\right\}$.

(2) $H_{\phi}$ est, par définition, l'ensemble des $\psi \in C^{\infty}$ qui sont de la forme

$$
\psi(x)=\left(\zeta, U_{x} \phi\right) \quad \text { où } \zeta \in \mathfrak{H}_{\phi} .
$$

Si $\left[\zeta_{1}, \zeta_{2}\right]$ est l'image de $\zeta$ dans l'isomorphisme qui applique $\left\{\mathfrak{C}_{\phi}, U_{x}, \phi\right\}$ sur $\left\{\right.$ FC, $\left.U_{x},\left[\phi_{1}, \phi_{2}\right]\right\}$ on a donc

$$
\begin{aligned}
\psi(x) & =\left(\left[\zeta_{1}, \zeta_{2}\right], U_{x}\left[\phi_{1}, \phi_{2}\right]\right)=\left(\zeta_{1}, U_{x} \phi_{1}\right)+\left(\zeta_{2}, U_{x} \phi_{2}\right) \\
& =\psi_{1}(x)+\psi_{2}(x) \quad\left(\psi_{1} \in H_{\phi_{1}}, \psi_{2} \in H_{\phi_{2}}\right)
\end{aligned}
$$

d'où 


$$
H_{\phi} \subset H_{\phi_{1}}+H_{\phi_{2}} \text {. }
$$

Réciproquement, soient

$$
\psi_{1}(x)=\left(\zeta_{1}, U_{x} \phi_{1}\right), \quad \psi_{2}(x)=\left(\zeta_{2}, U_{x} \phi_{2}\right)
$$

deux éléments de $H_{\phi_{1}}, H_{\phi_{2}}$ respectivement. On a, en désignant par $\left[\xi_{1}, \xi_{2}\right]$ la projection orthogonale de l'élément $\left[\zeta_{1}, \zeta_{2}\right] \in \mathfrak{H}_{\phi_{1}} \times \mathfrak{H}_{\phi_{2}}$ sur $\mathfrak{H C}$ :

$$
\psi_{1}(x)+\psi_{2}(x)=\left(\left[\zeta_{1}, \zeta_{2}\right], U_{x}\left[\phi_{1}, \phi_{2}\right]\right)=\left(\left[\xi_{1}, \xi_{2}\right], U_{x}\left[\phi_{1}, \phi_{2}\right]\right)=\left(\xi, U_{x} \phi\right)
$$

où $\xi$ est l'image, dans $\mathfrak{K}_{\phi}$, de $\left[\xi_{1}, \xi_{2}\right] \in \mathfrak{C}$ par l'isomorphisme déja considéré.

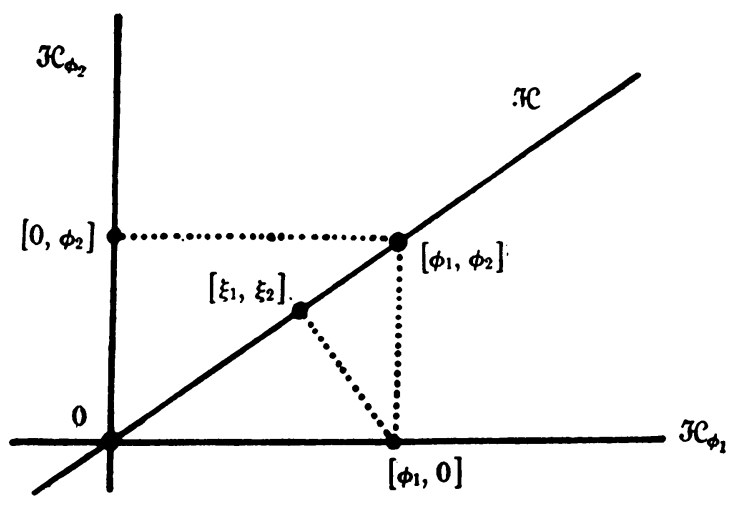

Fig. 1.

Donc $\psi_{1}+\psi_{2} \in H_{\phi}$, et finalement on a

$$
H_{\phi}=H_{\phi_{1}}+H_{\phi_{2}}
$$

comme annoncé.

Conséquences $d u$ Theorème 4 . Soit $\phi$ une fonction continue de type positif; si $\phi_{1} \ll \phi$, on a $\phi=\phi_{1}+\phi_{2}$ où $\phi_{2} \in \mathcal{P}$ : donc $H_{\phi_{1}} \subset H_{\phi}$ et en particulier $\phi_{1} \in H_{\phi}$. On peut donc écrire

$$
\phi_{1}(x)=\left(\xi, U_{x} \phi\right)
$$

où $\xi$ est un élément de $\mathfrak{H}_{\phi}$ qu'on peut obtenir comme suit: dans $\mathfrak{H}_{\phi_{1}} \times \mathfrak{H}_{\phi_{2}}$, soi $\left[\xi_{1}, \xi_{2}\right]$ la projection orthogonale de $\left[\phi_{1}, 0\right]$ sur $\mathfrak{H C}$ (cf. fig. 1).

Alors on a $\phi_{1}(x)=\left(\left[\phi_{1}, 0\right], U_{x}\left[\phi_{1}, 0\right]\right)=\left(\left[\phi_{1}, 0\right], U_{x}\left[\phi_{1}, \phi_{2}\right]\right)=\left(\left[\xi_{1}, \xi_{2}\right]\right.$, $\left.U_{x}\left[\phi_{1}, \phi_{2}\right]\right): \xi$ est donc l'image dans $\mathfrak{H C}_{\phi}$ de l'élément $\left[\xi_{1}, \xi_{2}\right] \in \mathfrak{H C}$. Il en résulte notamment que

$$
|\xi| \leqq|\phi| \text {. }
$$

L'ensemble des $\xi \in \mathcal{H}_{\phi}$ ainsi obtenus (et caractérisés par le fait que la fonction

$$
\psi(x)=\left(\xi, U_{x} \phi\right)
$$


est de type positif et $\ll \phi$ ) forme évidemment une partie convexe, fermée et bornée de $\mathfrak{H}_{\phi}$-partie non vide, d'ailleurs, puisqu'elle contient au moins les $\lambda \phi(0 \leqq \lambda \leqq 1)$.

11. Minorantes et opérateurs dans $\mathfrak{K}_{\phi}$. L'importance d'une étude des "minorantes" d'une $\phi \in \Phi$ est que cette étude conduit-et est même équivalente-à celle des opérateurs bornés de $\mathfrak{H}_{\phi}$ permutables aux $U_{x}$. On a en effet le

THEOREME 5. Il existe une correspondance biunivoque entre les $\psi \in \mathcal{P}$ "majorées" par une fonction donnée $\phi \in \mathcal{P}$, et les opérateurs $A$ hermitiens positifs, bornés par 1, définis dans $\mathfrak{\Im C}_{\phi}$, et permutables aux $U_{x}$; si de plus $A$ est associé a $\psi$, on a dans $H_{\phi}$

$$
\psi=A \phi .
$$

Démonstration. (1) Soit, dans $\mathfrak{C}_{\phi}, A$ un opérateur hermitien, positif, majoré par 1, et permutable aux $U_{x}$. Posons

$$
\psi(x)=\left(A \phi, U_{x} \phi\right) .
$$

Alors on a

$$
\begin{aligned}
\sum_{i, j} \alpha_{i} \bar{\alpha}_{j} \psi\left(s_{i}^{-1} s_{j}\right) & =\sum \alpha_{i} \bar{\alpha}_{j}\left(A \phi, U_{s_{i}^{-1} s_{j} \phi}\right)=\sum \alpha_{i} \bar{\alpha}_{j}\left(U_{s_{i}} A \phi, U_{s_{j}} \phi\right) \\
& =\sum \alpha_{i} \bar{\alpha}_{j}\left(A U_{s_{i}} \phi, U_{s_{j}} \phi\right)=\left(A \sum_{i} \alpha_{i} U_{s_{i}} \phi, \sum_{i} \alpha_{i} U_{s_{i} \phi}\right)
\end{aligned}
$$

d'où, avec

$$
0 \leqq(A \xi, \xi) \leqq(\xi, \xi)
$$

résulte

$$
0 \leqq \sum \alpha_{i} \bar{\alpha}_{j} \psi\left(s_{i}^{-1} s_{j}\right) \leqq \sum \alpha_{i} \bar{\alpha}_{j} \phi\left(s_{i}^{-1} s_{j}\right) ;
$$

c'est à dire $\psi \in \mathcal{P}, \psi \ll \phi$.

En observant que $\psi(x) \equiv 0$ entraine, d'après ce qui précède,

$$
(A \xi, \xi)=0
$$

pour les

$$
\xi=\sum \alpha_{i} U_{s_{i} \phi}
$$

denses dans $\mathfrak{H}_{\phi}$ et donc: entraine $A=0$-on voit que l'application $A \rightarrow \psi$ est biunivoque.

(2) Soit réciproquement une $\psi \in \Phi$ avec $\psi \ll \phi$; on a

$$
\psi(x)=\left(\zeta, U_{x} \phi\right)=\overline{\psi\left(x^{-1}\right)}=\left(\phi, U_{x} \zeta\right)
$$

où $\zeta$ est bien déterminé dans $\mathfrak{T}_{\phi} . \mathrm{Si}$ 


$$
\xi=\sum \alpha_{i} U_{s_{i} \phi}
$$

et si l'on pose

$$
\eta=\sum \alpha_{i} U_{s_{i}} \zeta
$$

on a alors

$$
\begin{aligned}
\left(\xi, U_{x} \zeta\right) & =\left(\sum \alpha_{i} U_{s_{i} \phi}, U_{x} \zeta\right)=\sum \alpha_{i}\left(\phi, U_{s_{i}^{-1}} \zeta\right) \\
& =\sum \alpha_{i}\left(\zeta, U_{s_{i}^{-1} x^{-1}}\right)=\left(\sum \alpha_{i} U_{s_{i}} \zeta, U_{x} \phi\right) \\
& =\left(\eta, U_{x} \phi\right):
\end{aligned}
$$

en sorte que $\xi=0$ entraine $\left(\eta, U_{x} \phi\right)=0$ et donc $\eta=0$. L'application $\xi \rightarrow \eta$ est donc linéaire et biunivoquè: De plus on a

$$
(\eta, \xi)=\left(\sum \alpha_{i} U_{s_{i}} \xi, \sum \alpha_{j} U_{s_{j}} \phi\right)=\sum \alpha_{i} \bar{\alpha}_{j} \psi\left(s_{i}^{-1} s_{j}\right)
$$

d'où, d'après $0 \ll \psi \ll \phi$ :

$$
0 \leqq(\eta, \xi) \leqq(\xi, \xi):
$$

on peut donc poser

$$
\eta=A \xi
$$

où $A$ est hermitien positif, majoré par 1 , et donc prolongeable à tout $\mathfrak{H}_{\phi}$.

De plus on a

$$
A U_{x} \xi=A U_{x}\left(\sum \alpha_{i} U_{s_{i}} \phi\right)=A\left(\sum \alpha_{i} U_{x s_{i}} \phi\right)=\sum \alpha_{i} U_{x s_{i}} \zeta=U_{x} A \xi
$$

d'où la permutabilité de $A$ et $U_{x}$ : ce qui démontre le théorème.

Conséquence $d u$ Théorème 5. (1) Soit $0 \ll \psi \ll \phi$; soit $A$ l'opérateur associé, dans $H_{\phi}$, à $\psi$; soit $B$ la racine carrée positive de $A$ : comme $A, B$ est permutable aux $U_{x}$. On peut donc écrire

$$
\psi(x)=\left(A \phi, U_{x} \phi\right)=\left(B^{2} \phi, U_{x} \phi\right)=\left(\zeta, U_{x} \zeta\right)
$$

où $\zeta=B \phi$. Cette représentation met en évidence le caractère "positif" de $\psi$; et surtout, du fait qu'on peut approcher fortement $\zeta$ par des $\sum \alpha_{i} U_{s_{i}} \phi$, prouve le

Theoreme 6. Toute fonction $\psi \in \mathcal{P}$ majorée par $\phi \in \mathcal{P}$ est limite, uniforme sur $G$, de fonctions (de $P$ ) de la forme

$$
\sum_{i, j} \alpha_{i} \bar{\alpha}_{j} \phi\left(s_{i}^{-1} x s_{j}\right) .
$$

Il est clair que ce résultat subsiste si, plus généralement, il existe une constante positive $n<+\infty$ telle que $\psi \ll n \phi$. (2) Supposons la structure $\left\{\mathcal{H}_{\phi}, U_{x}\right\}$ irreductible: on dira que $\phi$ est elémentaire. Comme alors les seuls opérateurs permutables aux $U_{x}$ sont les multiples de l'unité, et comme on peut évidem- 
ment se borner aux opérateurs hermitiens positifs, on a le

Theoreme 7. Pour qu'une $\phi \in P$ soit élémentaire, il faut et il suffit que toute minorante de $\phi$ dans $\Phi$ soit de la forme $\lambda \phi(0 \leqq \lambda \leqq 1)$.

Nota. Les Théorèmes 5 et 7 sont dûs à Gelfand-Raīkov. Ils ont été retrouvés indépendamment par l'auteur, ainsi que les autres résultats de ce §.

12. Parties disjointes $d^{\prime}$ une fonction de type positif. La notion de "parties disjointes" d'une $\phi \in P$, qu'on va développer dans cette section, éclairera le Théorème 4. Soit

$$
\phi=\phi_{1}+\phi_{2}
$$

une partition de $\phi$. On a vu au no. 1 que la structure unitaire simple $\left\{\mathfrak{C}_{\phi}, U_{x}, \phi\right\}$ était isomorphe à $\left\{\mathfrak{C}, U_{x},\left[\phi_{1}, \phi_{2}\right]\right\}$. On peut se demander à quelle condition $\mathfrak{H C}$ est formée par tout le produit $\mathfrak{F C}_{\phi_{1}} \times \mathfrak{K C}_{\phi_{2}}$. Pour cela, il faut et il suffit que tout élément $\left[\xi_{1}, \xi_{2}\right] \in \mathfrak{F}_{\phi_{1}} \times \mathfrak{H}_{\phi_{2}}$ orthogonal à $\mathfrak{H}$ soit nul. Or l'hypothèse faite équivaut à

$$
\left(\left[\xi_{1}, \xi_{2}\right], U_{x}\left[\phi_{1}, \phi_{2}\right]\right) \equiv 0
$$

c'est à dire, en appelant $\psi_{1}(x)$ et $\psi_{2}(x)$ les images dans $H_{\phi_{1}}$ et $H_{\phi_{2}}$ des éléments $\xi_{1}$ et $\xi_{2}$ de $\mathfrak{H}_{\phi_{1}}$ et $\mathfrak{H}_{\phi_{2}}$, à $\psi_{1}(x)+\psi_{2}(x) \equiv 0$. La condition cherchée est donc que

$$
\left.\begin{array}{l}
\psi_{1} \in H_{\phi_{1}} \\
\psi_{2} \in H_{\phi_{2}} \\
\psi_{1}+\psi_{2}=0
\end{array}\right\} \quad \text { entraine } \psi_{1}=\psi_{2}=0
$$

-en d'autres termes: que $H_{\phi_{1}} \cap H_{\phi_{2}}$ se réduise à $\{0\}$. Si cette condition est remplie, nous disons que $\phi_{1}$ et $\phi_{2}$ sont disjointes-et que $\phi_{1}$ (ou $\phi_{2}$ ) est une partie disjointe de $\phi$. On a alors $H_{\phi}=H_{\phi_{1}} \oplus H_{\phi_{2}}$ le signe $\oplus$ indiquant, comme d'habitude, la somme directe de deux souse-spaces vectoriels supplémentaires.

De plus, $\left\{\mathfrak{C}_{\phi}, U_{x}\right\}$ est isomorphe au produit des structures

$$
\left\{\mathfrak{C}_{\phi_{1}}, U_{x}\right\} \text { et }\left\{\mathfrak{K}_{\phi_{9},}, U_{x}\right\} \text {. }
$$

Il en résulte inversement que l'on peut trouver dans $\mathcal{H}_{\phi}$ deux variétés linéaires fermées invariantes $W_{1}$ et $W_{2}$ telles que:

$$
\begin{aligned}
\mathfrak{H}_{\phi} & =W_{1} \oplus W_{2}, \\
\phi_{1}(x) & =\left(\zeta_{1}, U_{x} \phi\right)=\left(\zeta_{1}, U_{x} \zeta_{1}\right), \\
\phi_{2}(x) & =\left(\zeta_{2}, U_{x} \phi\right)=\left(\zeta_{2}, U_{x} \zeta_{2}\right)
\end{aligned}
$$

où $\zeta_{1}$ et $\zeta_{2}$ sont les projections orthogonales de $\phi$ sur $W_{1}$ et $W_{2}$.

Réciproquement soit $\mathfrak{H}_{\phi}=W_{1} \oplus W_{2}$ une "décomposition" de $\mathfrak{H C}_{\phi}$ en deux variétés linéaires fermées invariantes supplémentaires; soient $\zeta_{1}$ et $\zeta_{2}$ les projections de $\phi$ sur $W_{1}$ et $W_{2}$. On a 


$$
\begin{aligned}
\phi(x) & =\left(\phi, U_{x} \phi\right)=\left(\zeta_{1}+\zeta_{2}, U_{x}\left(\zeta_{1}+\zeta_{2}\right)\right) \\
& =\left(\zeta_{1}, U_{x} \zeta_{1}\right)+\left(\zeta_{2}, U_{x} \zeta_{2}\right)=\phi_{1}(x)+\phi_{2}(x)
\end{aligned}
$$

d'où une partition de $\phi$; de plus, $\phi_{1}$ et $\phi_{2}$ sont disjointes, car toute $\psi_{1} \in H_{\phi_{1}}$ est de la forme

$$
\left(\zeta_{1}^{\prime}, U_{x} \zeta_{1}\right)=\left(\zeta_{1}^{\prime}, U_{x} \phi\right) \quad\left(\zeta_{1}^{\prime} \in W_{1}\right)
$$

et toute $\psi_{2} \in H_{\phi_{2}}$ de la forme

$$
\left(\zeta_{2}^{\prime}, U_{x} \zeta_{2}\right)=\left(\zeta_{2}^{\prime}, U_{x} \phi\right)
$$

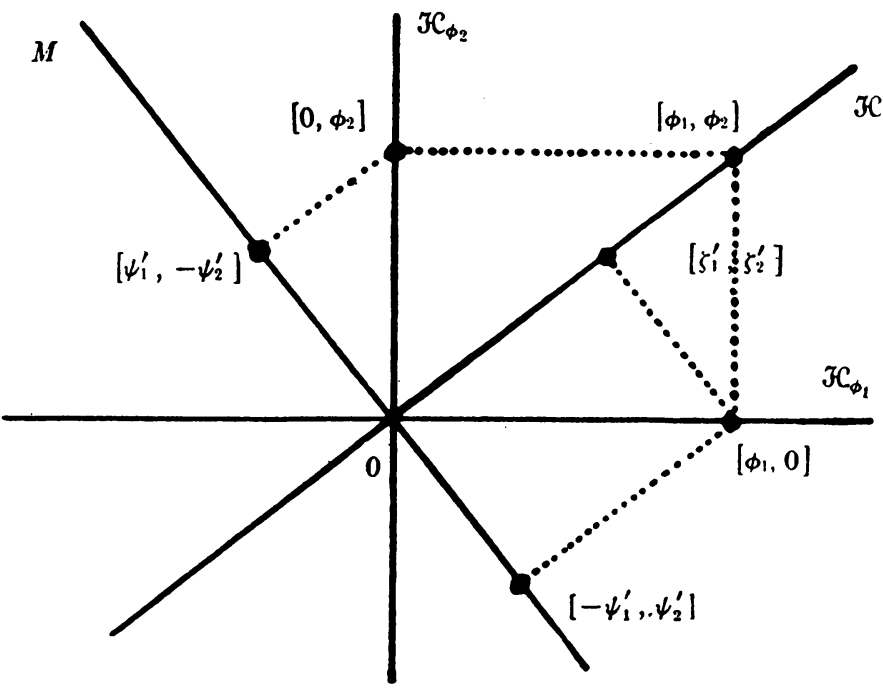

FIG. 2.

en sorte que $H_{\phi_{1}}$ et $H_{\phi_{2}}$ sont les images dans $H_{\phi}$ de $W_{1}$ et $W_{2}$, et vérifient donc $H_{\phi_{1}} \cap H_{\phi_{2}}=\{0\}$.

Le fait que deux fonctions $\phi_{1}, \phi_{2} \in P$ sont disjointes peut s'exprimer uniquement à l'aide de la relation d'ordre dans $\boldsymbol{P}$. Observons d'abord que, dans ce cas, toute $\psi \in \mathcal{P}$ qui minore à la fois $\phi_{1}$ et $\phi_{2}$ est nulle: car d'après le Théorème 4 on $a$

$$
\psi \in H_{\phi_{1}} \cap H_{\phi_{2}} .
$$

Réciproquement, si 0 est la seule minorante de $\phi_{1}$ et $\phi_{2}$, celles-ci sont disjointes. Sinon soient, dans $\mathfrak{H}_{\phi_{1}} \times \mathfrak{H}_{\phi_{2}}, \mathfrak{H C}$ la variété linéaire fermée invariante engendrée par $\left[\phi_{1}, \phi_{2}\right]$, et $M$ la variété supplémentaire de $\mathfrak{H C}$ (fig. 2): $M$ est invariante et, si $\phi_{1}$ et $\phi_{2}$ ne sont pas disjointes, n'est pas réduite à zéro. Soit alors $\left[-\psi_{1}^{\prime}, \psi_{2}^{\prime}\right]$ la projection orthognale de $\left[\phi_{1}, 0\right]$ sur $M .\left[-\psi_{1}^{\prime}, \psi_{2}^{\prime}\right]$ n'est pas nul-sinon on aurait $M \subset \mathfrak{H C}_{\phi_{2}}$ donc $\mathcal{H C C H}_{\phi_{1}}$ contrairement au fait que 
$\phi_{2} \neq 0$. D'autre part, la projection de $\left[0, \phi_{2}\right]$ sur $M$ est évidemment $\left[\psi_{1}^{\prime},-\psi_{2}^{\prime}\right]$ et l'on a

$$
\begin{aligned}
\left(\left[-\psi_{1}^{\prime}, \psi_{2}^{\prime}\right], U_{x}\left[-\psi_{1}^{\prime}, \psi_{2}^{\prime}\right]\right) & =\left(\psi_{1}^{\prime}, U_{x} \psi_{1}^{\prime}\right)+\left(\psi_{2}^{\prime}, U_{x} \psi_{2}^{\prime}\right) \\
& =\left(\left[-\psi_{1}^{\prime}, \psi_{2}^{\prime}\right], U_{x}\left[\phi_{1}, 0\right]\right)=-\psi_{1}(x)
\end{aligned}
$$

et de même

$$
=\left(\left[-\psi_{1}^{\prime}, \psi_{2}^{\prime}\right], U_{x}\left[0, \phi_{2}\right]\right)=\psi_{2}(x) ;
$$

enfine, si $\left[\zeta_{1}^{\prime}, \zeta_{2}^{\prime}\right]$ est la projection de $\left[\phi_{1}, 0\right]$ sur $\mathfrak{F C}$, on aura

$$
\begin{aligned}
\phi_{1}(x)= & \left(\left[\phi_{1}, 0\right], U_{x}\left[\phi_{1}, 0\right]\right)=\left(\left[\zeta_{1}^{\prime}, \zeta_{2}^{\prime}\right], U_{x}\left[\zeta_{1}^{\prime}, \zeta_{2}^{\prime}\right]\right) \\
& +\left(\left[-\psi_{1}^{\prime}, \psi_{2}^{\prime}\right], U_{x}\left[-\psi_{1}^{\prime}, \psi_{2}^{\prime}\right]\right)
\end{aligned}
$$

en sorte que la fonction $-\psi_{1} \in \mathcal{P}$ minore $\phi_{1}$; on verrait de même qu'elle minore $\phi_{2}:$ or $\psi_{1}(x)$ n'est pas nulle. La proposition est donc démontrée, et on a en définitive le

THEOREME 8. Etant données deux fonctions $\phi_{1}, \phi_{2} \in \mathcal{P}$, les propositions suivantes sont équivalentes:

(a) $H_{\phi_{1}} \cap H_{\phi_{2}}=\{0\}$;

(b) 0 est la seule minorante commune à $\phi_{1}$ et $\phi_{2}$;

(c) Les structures unitaires simples $\left\{\mathfrak{F}_{\phi_{1}}, U_{x}, \phi_{1}\right\}$ et $\left\{\mathfrak{H}_{\phi_{2}}, U_{x}, \phi_{2}\right\}$ sont contenues dans $\left\{\mathcal{H}_{\phi}, U_{x}, \phi\right\}$ où $\phi(x)=\phi_{1}(x)+\phi_{2}(x)$;

(d) $\left\{H_{\phi}, U_{x}\right\}$ est isomorphe da $\left\{\mathfrak{F}_{\phi_{1}}, U_{x}\right\} \times\left\{\mathfrak{K}_{\phi_{2}}, U_{x}\right\}$.

La propriété (b) justifie le mot "disjointe," qui a été introduit par F. Riesz $\left.{ }^{21}\right)$ dans la théorie des groupes abéliens ordonnés. La propriété (c) montre que toute partie disjointe d'une $\phi \in \mathcal{P}$ est déterminée par un sous espace invariant fermé de $\mathfrak{K}_{\phi}$, et réciproquement.

Terminons en indiquant comment toute minorante $\psi$ de $\phi$ peut être reconstituée avec des parties disjointes de $\phi$. Soit $A$ l'opérateur associé à $\psi$ dans $\mathfrak{H}_{\phi}$; soit

$$
A=\int_{0}^{1} \lambda d E_{\lambda}
$$

sa décomposition spectrale. Comme $A$ permute aux $U_{x}$, il en est de même des projecteurs $E_{\lambda}$, qui sont donc associés à des variétés $V_{\lambda}$ invariantes dans $\mathfrak{H}_{\phi}$. D'où résulte que

$$
e_{\lambda}=E_{\lambda} \phi \in H_{\phi}
$$

(21) F. Riesz, loc. cit. (Ann. of Math. (1940)). 
est une partie disjointe de $\phi$; mais on a

$$
\psi(x)=\left(A \phi, U_{x} \phi\right)
$$

d'où

$$
\psi(x)=\int_{0}^{1} \lambda d\left(E_{\lambda} \phi, U_{x} \phi\right)=\int_{0}^{1} \lambda d e_{\lambda}(x):
$$

on a ainsi exprimé $\psi$ à l'aide de parties disjointes de $\phi$. Bien entendu, les $e_{\lambda}$ forment, dans $P$, une famille "croissante", etc. Nous n'insisterons pas ici sur ces questions dont, jusqu'à nouvel ordre, l'intérêt est de simple curiosité (bien que ces propriétés correspondent probablement à une "dualité" non abélienne qui reste encore à faire). Naturellement, la "décomposition spectrale" qui précède permet de donner une démonstration du Théorème 7 .

\section{B. TheOREMES FondamentauX D'APPROXIMATION}

13. Le théorème de Krein et Milman. Cette section est déstinée à rappeler quelques propriétes $\left({ }^{22}\right)$ - dont toutes ne sont pas classiques-des espaces de Banach réels.

(a) Soit $E$ un tel espace, d'éléments $x, y, \ldots$, où l'on notera $\|x\|$ la norme. Une fonctionnelle linéaire bornée sur $E$ est une fonction $\mu(x)$ à valeurs réelles, et vérifiant

(1) $\mu(x+y)=\mu(x)+\mu(y)$,

(2) $|\mu(x)| \leqq M \cdot\|x\|$.

$\mathrm{La}$ "norme" de cette fonctionnelle sera le plus petit $M$ vérifiant 2 -on la notera $\|\mu\|$, et on aura

$$
\|\mu\|=\inf _{x \neq 0} \frac{|\mu(x)|}{\|x\|} .
$$

L'ensemble de ces $\mu$ ainsi normées forme évidemment à son tour un espace de Banach $E^{\prime}$-le dual de $E$. On notera maintenant $x^{\prime}, y^{\prime}, \cdots$ les éléments de $E^{\prime}$, et $\left(x, x^{\prime}\right)$ la valeur en $x \in E$ de la fonctionnelle $x^{\prime} \in E^{\prime}$; on a donc

$$
\left|\left(x, x^{\prime}\right)\right| \leqq\|x\| \cdot\left\|x^{\prime}\right\| .
$$

(b) La topologie faible dans $E^{\prime}$ est définie comme étant la moins fine pour laquelle $\left(x, x^{\prime}\right)$ est, pour chaque $x \in E$, une fonction continue de $x^{\prime}$.

Une partie $A^{\prime} \subset E^{\prime}$ sera dite bornée si l'on a

$$
\sup _{x^{\prime} \in A^{\prime}}\left\|x^{\prime}\right\|<+\infty \text {. }
$$

Sur une telle partie, la topologie faible ne change pas si, pour la définir, on

(22) La terminologie adoptée ici est celle de J. Dieudonné, La dualité dans les espaces vectoriels topologiques, Ann. Ecole Norm. 
substitue à $E$ un ensemble dense dans $E$. De plus, on a le

LEMME A. Toute partie bornée et faiblement fermée de $E^{\prime}$ est compacte pour la topologie faible.

C'est le cas, notamment, de la "boule unité" qui se compose des $x^{\prime} \in E^{\prime}$ vérifiant $\left\|x^{\prime}\right\| \leqq 1$.

(c) Soit $K^{\prime}$ un ensemble non vide, borné, convexe, et faiblement fermé dans $E^{\prime}$. Pour chaque $x \in E$ posons

$$
\sup _{x^{\prime} \in K^{\prime}}\left(x, x^{\prime}\right)=M_{x} \quad(<+\infty) .
$$

$\left(x, x^{\prime}\right)$ étant une fonction continue $\left.{ }^{23}\right)$ sur le compact $K^{\prime}$, y atteint effectivement son maximum.

Lemme B. $L^{\prime}$ "hyperplan" $H_{x}$ lieu des $x^{\prime} \in E^{\prime}$ tels que

$$
\left(x, x^{\prime}\right)=M_{x}
$$

est faiblement fermé et rencontre $K^{\prime}$; de plus, $K^{\prime}$ est l'intersection (quand $x$ parcourt $E$ ) des "demi-espaces" lieux des $x^{\prime} \in E^{\prime}$ tels que

$$
\left(x, x^{\prime}\right) \leqq M_{x} \text {. }
$$

Les hyperplans $H_{x}$ seront appelés hyperplans d'appui de $K^{\prime}$.

(d) $K^{\prime}$ ayant les mêmes propriétés qu'en (c), on dira qu'un $x^{\prime} \in K^{\prime}$ est un point extrémal de $K^{\prime}$ s'il n'est strictement intérieur à aucun segment de droite dans $K^{\prime}$-en d'autres termes si

$$
x^{\prime}=\lambda y^{\prime}+\mu z^{\prime} \quad\left(y^{\prime}, z^{\prime} \in K^{\prime} ; 0 \leqq \lambda, \mu \leqq 1 ; \lambda+\mu=1\right)
$$

exige $y^{\prime}=x^{\prime}$ ou $z^{\prime}=x^{\prime}$. On a au sujet de ces ensembles le

Theoreme de Krein et Milman. Le plus petit ensemble convexe faiblement fermé contenant les points extrémaux. de $K^{\prime}$ est $K^{\prime}$ luimème.

Démonstration. Soit $K^{\prime}$ un ensemble convexe, faiblement borné et faiblement fermé dans $E^{\prime}$. Nous appelerons variêté d'appui de $K^{\prime}$ tout ensemble $V^{\prime} \subset E^{\prime}$ possèdant les propriétés suivantes:

(1) $V^{\prime} \cap K^{\prime}$ n'est pas vide;

(2) si $x^{\prime} \in V^{\prime} \cap K^{\prime}$, tout segment de $K^{\prime}$ auquel $x^{\prime}$ est interieur est contenu dans $V^{\prime}$;

(3) $V^{\prime}$ est l'ensemble des $x^{\prime} \in E^{\prime}$ qui vérifient les équations

$$
\left(x_{i}, x^{\prime}\right)=a_{i}
$$

où les $x_{i}$ sont des points déterminés de $E$ (non necessairement en nombre fini), et les $a_{i}$ des constantes réelles également déterminées.

(29) Ici comme toujours dans la suite-sauf avis contraire-les notions topologiques sont prises au sens de la topologie faible de $E^{\prime}$. 
(1) Existence des variétés d'appui: il est clair que les "hyperplans d'appui" définis en (c) appartiennent à cette catégorie; réciproquement, toute variété d'appui définie par une seule équation ( $\alpha$ ) est un hyperplan d'appui.

Il est clair aussi que les points extrémaux de $K^{\prime}$ (s'ils existent) ne sont autres que les variétés d'appui de dimension zéro.

(2) Tout ordonné filtrant décroissant de variêtés d'appui a pour intersection une variété d'appui. Soit $V_{\alpha}^{\prime}$ un ordonné filtrant décroissant de variétés d'appui : quels que soient $\alpha, \beta$, il existe $\gamma$ tel que

$$
V_{\gamma}^{\prime} \subset V_{\alpha}^{\prime} \cap V_{\beta}^{\prime} \text {. }
$$

Notons $K_{\alpha}^{\prime}=K^{\prime} \cap V_{\alpha}^{\prime} ; K_{\alpha}^{\prime}$ est une partie non vide et fermée du compact $K^{\prime}$; de plus on a

$$
K_{\gamma}^{\prime} \subset K_{\alpha}^{\prime} \cap K_{\beta}^{\prime} .
$$

Les $K_{\alpha}^{\prime}$ forment ainsi un ordonné filtrant décroissant sur $K^{\prime}$. Ils ont donc au moins (Borel-Lebesgue pour les fermés) un point commun. Par suite, l'intersection de $K^{\prime}$ et de $V^{\prime}=\bigcap_{\alpha} V_{\alpha}^{\prime}$ n'est pas vide: condition (1).

Quant à la condition (2): si $x^{\prime} \in V^{\prime} \cap K^{\prime}$ est intérieur à $\left[y^{\prime}, z^{\prime}\right], x^{\prime}$ appartenant à $V_{\alpha}^{\prime} \cap K^{\prime}$ on a

$$
\left[y^{\prime}, z^{\prime}\right] \subset V_{\alpha}^{\prime}
$$

quel que soit $\alpha$

et par suite

$$
\left[y^{\prime}, z^{\prime}\right] \subset V^{\prime} .
$$

Enfin la condition (3) est vérifiée trivialement pour $V^{\prime}$, puisqu'elle l'est pour les $V_{\alpha}^{\prime}$.

$V^{\prime}$ est donc bien une variété d'appui comme annoncé.

(3) Toute variêtê d'appui contient au moins un point extrémal de $K^{\prime}$. Soit en effet $V^{\prime}$ une variété d'appui de $K^{\prime}$. Considérons toutes les variétés d'appui contenues dans $V^{\prime}$, et ordonnons les par $C$ : la partie (2) de la démonstration prouve qu'on obtient un ensemble inductif(24) (c'est à dire: tout ensemble "totalement ordonné" de variétés d'appui admet une "borne inférieure," à savoir son intersection). D'où résulte, par le théorème de Zorn( $\left.{ }^{24}\right)$ (équivalent a l'axiome de Zermelo) l'existence d'au moins une variété d'appui minimale: c'est à dire d'une variété d'appui $W^{\prime} \subset V^{\prime}$ telle qu'il n'existe pas de variété d'appui strictement contenue dans $W^{\prime}$.

Reste à prouver que $W^{\prime}$ est de dimension 0 , c'est à dire réduite à un point (qui sera, alors, extremal pour $K^{\prime}$ ). Or soit $K^{\prime \prime}=W^{\prime} \cap K^{\prime}: K^{\prime \prime}$ est convexe, borné, faiblement fermé, et non vide d'après la condition (1) sur $W^{\prime}$. Si $W^{\prime}$ contenait plus d'un point-c'est à dire était de dimension $>0$-le raisonnement utilisé dans la lère partie de la démonstration prouverait

(24) N. Bourbaki, Theorie des ensembles (Actualités Scientifiques et Industrielles, no. 846), Paris, 1939, p. 39. 
l'existence d'un hyperplan d'appui $H_{0}^{\prime}$ de $K^{\prime \prime}$, qu'on pourait choisir différent de $W^{\prime}$ (puisqu'on peut partir d'un $x_{0}$ arbitraire de $E$ ). Mais alors $W^{\prime \prime}=H_{0}^{\prime} \cap W^{\prime}$ serait une variété d'appui $d e K^{\prime}$ : en effet, les conditions (1) et (3) sont remplies pour $K^{\prime}$; quant à la condition 2 : si $x^{\prime} \in W^{\prime \prime} \cap K^{\prime}$ est intérieur au segment $\left[y^{\prime}, z^{\prime}\right] \subset K^{\prime}$, comme $W^{\prime \prime}$ est contenue dans la variété d'appui $W^{\prime}$ de $K^{\prime}$, on a aussi $x^{\prime} \in W^{\prime}$ et donc $\left[y^{\prime}, z^{\prime}\right] \subset W^{\prime} \cap K^{\prime}=K^{\prime \prime}$; or $x^{\prime}$ est dans la variêté d'appui $H_{0}^{\prime}$ de $K^{\prime \prime}$ : donc $\left[y^{\prime}, z^{\prime}\right] \subset H_{0}^{\prime}$-et finalement $\left[y^{\prime}, z^{\prime}\right] \subset W^{\prime} \cap H_{.0}^{\prime}=W^{\prime \prime}$, ce qui est bien la condition (2) pour $W^{\prime \prime}$ et $K^{\prime}$. Mais $W^{\prime}$ étant une variété d'appui minimale de $K^{\prime}$, on a alors $W^{\prime \prime}=W^{\prime}$ : d'où contradiction, ce qui prouve comme annoncé que $W^{\prime} \cap K^{\prime}$ se réduit à un point, extrémal pour $K^{\prime}$.

(4) $K^{\prime}$ est l'enveloppe convexe faiblement fermée de l'ensemble de ses points extrémaux. Soit en effet $K_{0}^{\prime}$ cette enveloppe: on a $K_{0}^{\prime} \subset K^{\prime}$. Si on avait $K_{0}^{\prime} \neq K^{\prime}$, on pourrait (puisque tout ensemble convexe faiblement fermé est l'intersection des demi-espaces qui le contiennent) trouver un $x_{0} \in E$ et un $x_{0}^{\prime} \in K^{\prime}-K_{0}^{\prime}$ avec $(*)\left(x_{0}, x^{\prime}\right) \leqq 0$ pour $x^{\prime} \in K_{0}^{\prime}$ et

$$
\left(x_{0}, x_{0}^{\prime}\right)>1 \text {. }
$$

Soit $M$ le maximum de $\left(x_{0}, x^{\prime}\right)$ pour $x^{\prime} \in K^{\prime}$ : on a $M \geqq 1$; soit $H_{0}^{\prime} l^{\prime}$ hyperplan défini par

$$
\left(x_{0}, x^{\prime}\right)=M \cap 1:
$$

la partie (1) de la démonstration prouve que $H_{0}^{\prime}$ est une variété d'appui de $K^{\prime}$-donc, d'après la partie (3), rencontre $K_{0}^{\prime}$-ce qui contredit $(*)$. Et le théorème est démontré.

Remarque. La question se pose de savoir si l'ensemble des points extrémaux d'une partie convexe bornée et faiblement fermée est lui-même faiblement fermé. Or, il n'en est rien comme le prouve l'exemple suivant (que M. Henri Cartan m'a communiqué): dans l'espace de Hilbert à base dénombrable (et qui est isomorphe à son dual) considérons le plus petit convexe fermé (fortement ou faiblement: les deux notions coincident pour les ensembles convexes d'un espace reflexif) contenant les points suivants (définis par leurs coordonnées relatives à une base orthogonale de l'espace):

$$
\begin{aligned}
& x_{1}: \quad-1,0,0,0, \cdots \text {, } \\
& x_{2}: \quad+1,0,0,0, \cdots \text {, } \\
& x_{3}: \quad 0,1 / 2,0,0, \cdots \text {, } \\
& x_{4}: \quad 0,0,1 / 4,0, \cdots \text {, } \\
& x_{5}: \quad 0, \quad 0, \quad 0,1 / 8, \cdots \text {, }
\end{aligned}
$$

Il est à peu près évident que les points $x_{n}$ sont des points extrémaux du convexe en question; or $x_{n}$ converge fortement vers 0 , qui appartient au convexe, 
et n'en est pas un point extrémal puisque

$$
0=\left(x_{1}+x_{2}\right) / 2 .
$$

Il serait désirable de trouver des critères simples permettant d'affirmer, dans certains cas, que les points extrémaux d'un convexe fermé forment euxmèmes un ensemble fermé.

On verra plus loin l'importance de ce problème pour la théorie des groupes.

14. Parties régulières de $P$. Pour appliquer les résultats obtenus dans les pages précédentes, nous allons considérer tout d'abord l'espace de Banach $E$ constitué par les fonctions numériques $f(x)$ vérifiant:

$$
\begin{aligned}
& \|f\|=\int|f(x)| \frac{d x}{(\rho(x))^{1 / 2}}<+\infty, \\
& f(x)=f^{\sim}(x)=\overline{f\left(x^{-1}\right)} .
\end{aligned}
$$

$E$ contient, entre autres, les fonctions de la forme

$$
f_{*} f^{\sim}(x)=\int f(x y) \overline{f(y)} d y \quad(f \in L) ;
$$

de plus, l'identité (I 7) montre aisément que toute $g \in L$ est limite, au sens de la norme (1), de combinaisons linéaires de fonctions de la forme (3).

Le dual réel $E^{\prime}$ de $E$ est évidemment formé des $\phi \in L^{\infty}$ telles que

$$
(f, \phi)=\int f(x) \phi(x) \frac{d x}{(\rho(x))^{1 / 2}}
$$

soit réel pour toute $f \in E$ : en d'autres termes, $E^{\prime}$ est constitué par les $\phi \in L^{\infty}$ qui vérifient

$$
\phi(x)=\phi^{\sim}(x)=\overline{\phi\left(x^{-1}\right)},
$$

presque-partout sur $G$ : ce qui est le cas des fonctions continues de type positif sur $G$ (rappelons que, d'après la proposition 4 , les seules fonctions de type positif contenues dans $L^{\infty}$ sont continues).

On peut donc identifier l'ensemble $P$ de ces fonctions à une partie de $E^{\prime}$.

De plus: $\phi \in \mathcal{P}$ équivaut (inégalité $\left(\mathrm{I}^{15^{\prime \prime}}\right)$ ) à

$$
\left(f * f^{\sim}, \phi\right)=\int f * f^{\sim}(x) \phi(x) \frac{d x}{(\rho(x))^{1 / 2}} \geqq 0
$$

pour toute $f \in L$. Il en résulte que $\mathcal{P}$ est une partie faiblement fermée de $E^{\prime}$. Nous noterons $\mathcal{P}_{0}$ l'ensembles des $\phi \in \mathcal{P}$ avec

$$
\|\phi\|_{\infty}=\phi(e) \leqq 1 \text {. }
$$


Comme la norme dans $E^{\prime}$, dual de $E$, coincide évidemment avec celle de $L^{\infty}$, on voit que $\mathcal{P}_{0}$ est une partie borneé de $E^{\prime}$; de plus, convexe trivialement; et, comme c'est l'intersection de deux ensembles faiblement fermés (à savoir $\boldsymbol{P}$ et la boule unité de $\left.E^{\prime}\right)$, faiblement fermée. C'est l'application du théorème de Krein et Milman à $\mathscr{P}_{0}$-et à des parties analogues à $\mathscr{P}_{0}$-qui donnera les résultats principaux de la théorie.

Notons encore le fait suivant: dans $\mathcal{P}_{0}$, la convergence faible de $\phi$ vers $\phi_{0}$ équivaut à la convergence vague ( $\S$ introductif) de la mesure $\phi(x) d x /(\rho(x))^{1 / 2}$ vers la mesure $\phi_{0}(x) d x /(\rho(x))^{1 / 2}$.

Definition 1. Une partie $A$ de $\mathcal{P}_{0}$ sera dite régulière si elle vérifie les conditions suivantes:

(1) A est convexe et faiblement fermée.

(2) A contient les "minorantes" de tous ses élêments.

(3) Si $\phi \in A, A$ contient aussi la fonction $\phi(x) / \phi(e)$.

Il est clair d'après (2) que $A$ contient 0 ; et que 0 est un point extrémal de $A$ - car si $\phi, \psi \in \mathbb{P}$ vérifient

$$
\phi+\psi=0, \text { on a } \phi(e)+\psi(e)=0 \text { d'où } \phi=\psi=0 .
$$

Proposition 6. Si A est une partie régulière de $\mathscr{P}_{0}$, tout point extrémal de $A$ autre que 0 est une $\phi(x)$ élémentaire vérifiant $\phi(e)=1$, et réciproquement.

(Ce théorème est dû à Gelfand et Raĩkov pour le cas où $A=\mathbb{P}_{0}$.)

Démonstration. Soit $\phi$ un point extrémal de $A, \phi \neq 0$. (a) Puisque $A$ contient 0 et $\phi / \phi(e)$, et que $\phi$ est sur le segment $[0, \phi / \phi(e)]$ de $A, \phi$ en est une extrémité: d'où $\phi(e)=1$.

(b) Soit $\psi \ll \phi$. D'après (2), $A$ contient $\psi$-donc aussi $\phi-\psi(\ll \phi)$-donc aussi, d'après (3):

$$
\xi=\frac{\psi}{\psi(e)}, \quad \eta=\frac{\phi-\psi}{\phi(e)-\psi(e)}=\frac{\phi-\psi}{1-\psi(e)} ;
$$

mais l'identité

$$
\phi=\psi(e) \cdot \xi+[1-\psi(e)] \cdot \eta
$$

prouve que $\phi$ est porté par le segment $[\xi, \eta]$ de $A$ : donc en est une extrémité, ce qui exige

$$
\phi=\xi=\frac{\psi}{\psi(e)} \quad \text { ou } \quad \phi=\eta=\frac{\phi-\psi}{1-\psi(e)}
$$

et dans tous les cas

$$
\psi=\phi \cdot \psi(e):
$$

donc $\psi \ll \phi$ entraine $\psi=\lambda \phi-$-et, d'après le théorème $7 ; \phi$ est élémentaire. 
(c) Réciproquement, soit $\phi$ une fonction élémentaire contenue dans $A$, et vérifiant $\phi(e)=1$. Supposons $\phi$ contenue dans le segment $[\psi, \theta]$ de $A$ :

$$
\phi=\alpha \cdot \psi+(1-\alpha) \theta \quad(0 \leqq \alpha \leqq 1) .
$$

On a $\alpha \psi \ll \phi$ : donc, si $\alpha \neq 0$, et $\phi$ étant élémentaire, on a

$$
\psi=\lambda \phi \quad(0 \leqq \lambda \leqq 1) .
$$

De même, si $\alpha \neq 1$, on a

$$
\theta=\mu \phi \quad(0 \leqq \mu \leqq 1) .
$$

Si donc on a $0<\alpha<1$ on en déduit

$$
\phi=\alpha \lambda \cdot \phi+(1-\alpha) \mu \cdot \phi
$$

d'où

$$
1=\alpha \lambda+(1-\alpha) \mu
$$

ce qui exige $\lambda=\mu=1$, et donc $\psi=\phi=\theta$.

Si au contraire $\alpha=0$ (ou $\alpha=1$ ), on a $\phi=\theta$ (ou $\phi=\psi$ ) -et dans tous les cas: $\phi$ est une extrémité du segment $[\psi, \theta]$, ce qui démontre la proposition.

Appliquant la proposition précédente et le théorème de Krein et Milmann à $\mathcal{P}_{0}$, on obtient le

TheOREme De Gelfand et Raİkov. Toute fonction continue de type positif $\phi(x)$ est limite faible, dans $L^{\infty}$, de fonctions de la forme

$$
\sum \alpha_{i} \cdot \phi_{i}(x) \quad\left(\alpha_{i} \geqq 0, \sum \alpha_{i} \leqq \phi(e)\right)
$$

où les $\phi_{i}$ sont des fonctions élémentaires égales à 1 en e.

Bien entendu, si $A$ est une partie régulière de $P_{0}$, le même théorème vaut pour $\phi \in A$ en prenant les $\phi_{i}$ dans $A$. Mais on va donner des résultats plus précis, qu'on obtiendra:

(1) en restreignant le plus possible les parties régulières considérées;

(2) en substituant, à la topologie faible de $L^{\infty}$, celle définie par la convergence uniforme sur toute partie compacte de $G$.

15. Le spectre d'une fonction de type positif. Soit $\phi(x)$ une fonction de type positif continue, avec $\phi(e) \leqq 1$. Nous désignerons par $A_{\phi}$ l'ensemble des fonctions de $\boldsymbol{P}_{0}$ qui sont de la forme

$$
\sum_{i, j} \alpha_{i} \bar{\alpha}_{j} \phi\left(s_{i}^{-1} x s_{j}\right)
$$

-ou sommes finies de telles fonctions; et par $\bar{A}_{\phi}$ l'ensemble des fonctions $d e \Phi_{0}$ quit sont approchables, uniformément sur toute partie compacte de $G$, par $\operatorname{des} \psi \in A_{\phi}$.

Proposition 7. $\bar{A}_{\phi}$ est une partie régulière de $\mathcal{P}_{0}$. 
Démonstration. (a) $A_{\phi}$ étant évidemment convexe, $\bar{A}_{\phi}$ l'est aussi. De plus, il est aussi trivial que

$$
\psi \in \bar{A}_{\phi} \text { entraine } \frac{\psi}{\psi(e)} \in \bar{A}_{\phi} \quad(\psi \neq 0) .
$$

(b) Soient $\psi \in \bar{A}_{\phi}$ et $\theta \ll \psi$; soient un compact $K \subset G$ et un $\epsilon>0$. D'après le Théorème 6 , on peut faire

$$
\left|\sum \beta_{i} \bar{\beta}_{j} \psi\left(t_{i}^{-1} x t_{j}\right)-\theta(x)\right| \leqq \epsilon \quad \text { pour tout } x \in G ;
$$

d'autre part, comme $\psi \in \bar{A}_{\phi}$, il existe $\phi^{\prime} \in A_{\phi}$ avec

$$
\left|\psi(x)-\phi^{\prime}(x)\right| \leqq \frac{\epsilon}{\sum\left|\beta_{i} \bar{\beta}_{i}\right|}
$$

dès que $x$ appartient au compact réunion des $t_{i}^{-1} K t_{j}$. Si alors $x \in K$ on a $\left|\sum \beta_{i} \bar{\beta}_{j} \psi\left(t_{i}^{-1} x t_{j}\right)-\sum \beta_{i} \bar{\beta}_{j} \phi^{\prime}\left(t_{i}^{-1} x t_{j}\right)\right|$

$$
\leqq \sum\left|\beta_{i} \bar{\beta}_{j}\right| \cdot\left|\psi\left(t_{i}^{-1} x t_{i}\right)-\phi^{\prime}\left(t_{i}^{-1} x t_{j}\right)\right| \leqq \epsilon
$$

d'où

$$
\left|\theta(x)-\sum \beta_{i} \bar{\beta}_{j} \phi^{\prime}\left(t_{i}^{-1} x t_{j}\right)\right| \leqq 2 \epsilon
$$

pour $x \in K$. Il reste à prouver que la fonction

$$
\phi^{\prime \prime}(x)=\sum \beta_{i} \bar{\beta}_{j} \phi^{\prime}\left(t_{i}^{-1} x t_{j}\right) \quad \text { est dans } A_{\phi}
$$

-ce qui se réduit à prouver qu'elle est $\leqq 1$ pour $x=e$ (car $\phi^{\prime}$ étant somme finie de fonctions

$$
\sum \alpha_{k} \bar{\alpha}_{h} \phi\left(s_{k}^{-1} x s_{h}\right),
$$

(1) sera somme finie de fonctions

$$
\left.\sum \alpha_{k} \beta_{i} \cdot \overline{\alpha_{h} \beta_{j}} \cdot \phi\left[\left(t_{i} s_{k}\right)^{-1} x\left(t_{j} s_{h}\right)\right]\right) .
$$

Mais il suffit pour cela de prendre pour $K$ un ensemble contenant $e$ : car on aura

$$
\left|\theta(e)-\phi^{\prime \prime}(e)\right| \leqq 2 \epsilon
$$

d'où

$$
\phi^{\prime \prime}(e) \leqq 1+2 \epsilon,
$$

et en remplacant au besoin $\phi^{\prime \prime} \operatorname{par} \phi^{\prime \prime} /(1+2 \epsilon)$ on ne sortira pas de $A_{\phi}$.

Par suite, $\bar{A}_{\phi}$ contient les minorantes de tous ses élements.

(c) Il reste à prouver que $\bar{A}_{\phi}$ est faiblement fermé. Nous allons montrer pour ceci-et c'est évidemment suffisant-que $\bar{A}_{\phi}$ n'est autre que l'adherence faible de $A_{\phi}$. Remarquons tout d'abord qu'on a la propriété suivante (qui n'est 
qu'un cas particulier du lemme de Gelfand sur la convergence faible des fonctionnelles linéaires):

Lemme C. Si une fonction varible $\phi \in \mathcal{P}_{0}$ converge faiblement vers une $\phi_{0} \in \mathcal{P}_{0}$, alors pour toute $f \in L$ la fonction ("régularisée de $\phi$ par f")

$$
\phi^{f}(x)=f * \phi * f^{\sim}(x)
$$

converge vers $\phi_{0}^{f}(x)$ uniformément sur toute partie compacte de $G$.

Soit alors une $\psi$ faiblement adhérente à $A_{\phi}$. Alors, pour toute $f \in L_{0}^{+}$, tout compact $K \subset G$ et tout $\epsilon>0$, existe une $\phi^{\prime} \in A_{\phi}$ avec

$$
\left|\psi^{f}(x)-\phi^{\prime f}(x)\right| \leqq \epsilon \quad \text { pour } x \in K .
$$

On peut de plus supposer

$$
\phi^{\prime f}(e) \leqq 1
$$

si on a

$$
\psi^{f}(e) \leqq 1 .
$$

Observons maintenant que dans ces conditions $\phi^{\prime \prime}(x)$ est dans $\bar{A}_{\phi}$ : il suffit pour le voir d'approcher étroitement la mesure $f(x) d x$ par des masses discrètes. Par suite, $\psi^{f}(x)$ est approchable à moins de $2 \epsilon \operatorname{sur} K \operatorname{par} \operatorname{des} \phi^{\prime \prime} \in A_{\phi}$. Mais $\psi$ est elle même approchable uniformément sur $G$ par ces $\psi^{\prime}(x)$ : et ainsi on obtient l'approximation cherchée, ce qui achève de démontrer la proposition.

Definition 2. On appelle spectre d'une fonction $\phi(x)$ continue de type positif l'ensemble des fonctions élémentaires $\psi(x)$ qui sont approchables, uniformément sur tout compact, par des fonctions de la forme

$$
\sum \alpha_{i} \bar{\alpha}_{j} \phi\left(s_{i}^{-1} x s_{j}\right)+\cdots+\sum \lambda_{k} \bar{\lambda}_{h} \phi\left(u_{k}^{-1} x u_{h}\right),
$$

et qui vérifient en outre $\psi(e)=1$.

DeFINITION 3. On appelle polynôme trigonométrique sur $G$ toute fonction de la forme

$$
a_{1} \phi_{1}(x)+\cdots+a_{n} \phi_{n}(x)
$$

où les $\phi_{i}(x)$ sont des fonctions élémentaires normées $\left({ }^{25}\right)$.

On va prouver maintenant le

THEOREME 9. Toute fonction continue et de type positif $\phi(x)$ est limite, uniforme sur tout compact, de polynômes trigonométriques $\psi(x)$, a coefficients positifs, constitués avec les éléments $d u$ spectre de $\phi$, et vérifiant $\psi(e)=\phi(e)$.

Démonstration. La proposition 6 prouve que $\phi$ (on suppose $\phi(e)=1$ ) est

(25) Une $\phi$ élémentaire sera dite normée si elle vérifie $\phi(e)=1$. 
limite faible dans $L^{\infty}$ de polynômes trigonométriques

$$
\psi(x)=\sum \alpha_{i} \phi_{i}(x) \quad\left(\alpha_{i} \geqq 0, \sum \alpha_{i} \leqq 1\right)
$$

où les $\phi_{i}$ sont dans le spectre de $\phi$. Il reste à transformer l'approximation faible en approximation uniforme sur tout compact. Pour cela, on remarque comme plus haut que, si $\psi \in \bar{A}_{\phi}$ et si $f \in L$, on a encore $\psi^{s} \in \bar{A}_{\phi}$-à condition que $\psi^{s}(e) \leqq 1$. On arrive ainsi à prouver que $\phi$ est limite, uniforme sur tout compact, de régularisées de polynômes trigonométriques. Le théorème est alors une conséquence immédiate de la

Proposition 8. (a) Si $\psi(x)$ est un polynôme trigonométrique et si $f \in L^{1}$, $f * \psi(x)$ est encore un polynôme trigonométrique; (b) si $\psi(x)$ est une fonction élémentaire sur $G$, il en est de même de

$$
\psi^{\mu}(x)=\iint \psi\left(s^{-1} x t\right) d \mu(s) \overline{d \mu(t)}
$$

quelle que soit la mesure $\mu$ de masse totale finie sur $G$.

Démonstration. (a) Se réduit à prouver que, si $\psi$ est elémentaire, $f * \psi$ est un polynôme trigonométrique.

Considérons dans $\mathcal{C}_{\psi}$ l'opérateur $T_{f}$ défini par

$$
\left(T_{f} \xi, \eta\right)=\int\left(U_{x} \xi, \eta\right) f(x) d x
$$

( $T_{f}$ est l'opérateur de Radon associé à la mesure $f(x) d x$ ); on aura

$$
f * \psi(y)=\int\left(U_{x} \psi, U_{y} \psi\right) f(x) d x=\left(T_{f} \psi, U_{y} \psi\right) .
$$

L'identité (I 7) montre alors que $f * \psi$ est une combinaison linéaire de fonctions de la forme

$$
\theta(x)=\left(\xi, U_{x} \xi\right)
$$

Mais $\left\{\mathcal{C}_{\psi}, U_{x}\right\}$ étant irréductible, $\left\{\mathcal{C}_{\theta}, U_{x}\right\}$, isomorphe au sous-espace de 3⿻ $\mathcal{C}_{\psi}$ engendré par les $U_{x} \xi$, l'est aussi: donc $\theta$ est élémentaire-et $f * \psi$ est un polynôme trigonométrique.

(b) Provient de ce que

$$
\psi^{\mu}(x)=\left(T_{\mu} \psi, U_{x} T_{\mu} \psi\right)
$$

définit une structure unitaire contenue dans $\left\{\mathfrak{C}_{\psi}, U_{x}\right\}$-donc elle-même irréductible (même raisonnement que précédemment pour $\theta$ ). Ajoutons que les mêmes raisonnements prouvent aussi la

Proposition 8. (c) Toute translatée (à gauche ou à droite) d'un polynôme trigonométrique est un polynôme trigonométrique. 
Revenons au spectre d'une $\phi \in \mathcal{P}_{0}$ : c'est l'ensemble des fonctions élémentaires normées (c'est à dire vérifiant $\psi(e)=1$ ) qu'on peut approcher, uniformément sur tout compact, par des sommes finies de fonctions de la forme

$$
\sum \alpha_{i} \dot{\alpha}_{j} \phi\left(s_{i}^{-1} x s_{j}\right) \text {. }
$$

Il est clair que si une fonction $\psi \in \mathcal{P}_{0}$ est dans $\bar{A}_{\phi}-$ c'est-à dire est elle-même approchable par de telles sommes finies-on a

$$
\bar{A}_{\psi} \subset \bar{A}_{\phi}
$$

et par suite: le spectre de $\psi$ est contenu dans celui de $\phi$. La réciproque de cette propriété est aussi valable d'après le Théorème 9 . En particulier, si une fonction $\psi$ est dans le spectre de $\phi$, il en est de même du spectre de $\psi$; notamment de toutes les fonctions (évidemment élémentaires et normées) de la forme

$$
\left(\xi, U_{x} \xi\right) \quad\left(\xi \in \mathfrak{S C}_{\psi},|\xi|=1\right) .
$$

A ce sujet, de nombreux problèmes, non encore résolus dans le cas général, se posent. Enonçons-en quelques uns.

Probleme 1. Soit $\phi(x)$ une fonction elémentaire. Le spectre de $\phi$ contient-il d'autres fonctions élémentaires normées que les

$$
\psi(x)=\left(\xi, U_{x} \xi\right) \quad \text { où } \xi \in \mathfrak{C}_{\phi}, \quad|\xi|=1 \text { ? }
$$

Problème 2. Si $\phi(x)$ est de type positif et limite faible de fonctions élémentaires normées, et n'est pas identiquement nulle, $\phi$ est-elle aussi élémentaire?

Problème 3. Dans l'ensemble des fonctions élémentaires normées, la convergence faible est-elle identique à la convergence uniforme sur tout compact?

Le problème 3 peut se résoudre dans le cas où $G$ possède un système fondamental de voisinages compacts de l'unité invariants par les automorphismes interieurs $\left(x \rightarrow s^{-1} x s\right)$ de $G$ (il suffit du reste de supposer l'existence d'un tel voisinage pour en déduire la propriété précédente; Weil, p. 129).

En effet, si $V$ est un tel voisinage, vérifiant $V=V^{-1}$, et si $f_{V}(x)$ est la fonction caractéristique de $V$, on a

$$
f_{V} \in E
$$

$$
f_{V}\left(s^{-1} x s\right) \equiv f_{V}(x) .
$$

De plus, si $\phi \in \mathcal{P}$ est $\neq 00$, il existe une telle $f_{V}$ avec

$$
\int f_{V}(x) \phi(x) \frac{d x}{(\rho(x))^{1 / 2}} \neq 0
$$

puisqu'on a $|\phi(x)|>\phi(e) / 2 \neq 0$ dans un voisinage convenable de $e$. Enfin, dans ce cas, $G$ est unimodulaire.

En effet, on a tout d'abord 


$$
\int f_{V}(x s) d x=\int f_{V}(s x) d x=\int f_{V}(x) d x ;
$$

d'autre part, les fonctions de la forme

$$
g(x)=\sum \alpha_{i} f_{V_{i}}\left(x s_{i}\right)
$$

sont denses dans l'espace $L^{1}$; sinon il existerait, d'après Hahn-Banach, une $\phi \in L^{\infty}$, non identiquement nulle, vérifiant

$$
\int f_{V}(x s) \phi(x) d x=\int f_{V}(s x) \phi(x) d x=0
$$

quels que soient $V$ et $s \in G$; d'où

$$
\int f_{V}(x) \phi(s x) d x \equiv 0
$$

et comme les $V$ sont "arbitrairement petits," $\phi=0$ (cf. la remarque terminant le $\S$ sur la régularisation). Or pour une telle $g \in L^{1}$ on a

$$
\int g(x s) d x=\int g(x) d x:
$$

donc cette égalité est valable pour toute $g \in L^{1}$, ce qui prouve bien $\rho(s) \equiv 1$.

Ceci étant, et si $\phi \in \mathcal{P}$, considérons l'opérateur $T_{f_{V}}$ défini dans $\mathfrak{H}_{\phi}$ par

$$
\left(T_{J_{V}} \xi, \eta\right)=\int\left(U_{\nu} \xi, \eta\right) f_{V}(y) d y .
$$

On a immédiatement

$$
\left(U_{x}^{-1} T_{f_{V}} U_{x} \xi, \eta\right)=\int\left(U_{x^{-1} y} \xi, \eta\right) f_{V}(y) d y=\int\left(U_{y} \xi, \eta\right) f_{V}\left(x y x^{-1}\right) d y=\left(T_{f_{V}} \xi, \eta\right)
$$

en sorte que $T_{f_{V}}$ permute aux $U_{2}$.

Si donc $\phi$ est élementaire on aura $T_{f_{V}}=\lambda \cdot 1$, et en particulier

$$
f_{V} * \phi(x)=\lambda \phi(x)
$$

où $\lambda$ est une constante égale à $f_{V} * \phi(e)$.

Ceci étant, supposons qu'une $\phi$ élémentaire normée converge faiblement vers une $\phi_{0}$ non identiquement nulle de $\mathcal{P}_{0}$; et choisissons $f_{V}$ de façon que

$$
\lambda_{0}=\int f_{V}(y) \phi_{0}\left(y^{-1}\right) d y=f_{V} * \phi_{0}(e) \neq 0 .
$$

Álors

(1) $f_{V} * \phi(x)=\lambda \phi(x)$ converge uniformément sur tout compact vers $f_{V} * \phi_{0}(x)$.

(2) $\lambda=f_{V} * \phi_{0}(e)$ tend vers $\lambda_{0}$ non nul. Donc $\phi=(1 / \lambda) f_{V} * \phi$ converge uniformément sur tout compact vers $\left(1 / \lambda_{0}\right) f_{V} * \phi_{0}$, nécessairement égal à $\phi_{0}$ puis- 
qu'en même temps $\phi$ converge faiblement vers $\phi_{0}$.

Ainsi dans ce cas, la convergence faible des fonctions élémentaires équivaut à leur convergence uniforme sur tout compact.

Les raisonnements précédents (qui reposent en somme sur l'existence du "centre" de l'anneau $L^{1}$ ) s'appliquent aux groupes abéliens, ou compacts, ou plus généralement représentables dans un groupe compact (Weil, p. 129), et, enfin, aux groupes discrets (mais dans ce cas le résultat obtenu est trivial, car les deux topologies sont identiques daǹs toute partie bornée de $L^{\infty}$ ).

En ce qui concerne le problème 2, sa solution est évidente si $G$ est abélien. On peut encore y répondre affirmativement dans le cas où $G$, non abélien, est compact; la solution repose sur les "relations d'orthogonalité" des coefficients des représentations irréductibles (cf. par exemple Weil, chap. V), et le résultat obtenu est le suivant, comme on le voit facilement: l'ensemble des fonctions élémentaires normées, muni de la topologie faible (ou forte-c'est équivalent d'après ce qui précède) de $L^{\infty}$ est localement compact; de plus, si $\phi$ est un élément de cet ensemble, la composante connexe de $\phi$ dans l'espace envisagé est l'ensemble des

$$
\psi(x)=\left(\xi, U_{x} \xi\right) \quad\left(\xi \in \mathcal{F}_{\phi}, \psi(e)=1\right)
$$

-c'est à dire le spectre de $\phi$.

Si $G$ est abélien, on retrouve ainsi le fait que le groupe dual de $G$ est discret. On verra du reste plus loin une proposition analogue pour les $G$ compacts non abéliens.

Enfin, le problème 1 est résolu affirmativement-et à peu près trivialement-si les représentations irréductibles de $G$ sont de dimension finie (ce qui exige que $G$ soit représentable dans un groupe compact; il est probable, quoique non démontré, que cette condition est aussi suffisante).

Avant de poursuivre l'étude des spectres, notons le

THEOREME 10. (a) Toute fonction continue sur $G$ est approchable, uniformément sur tout compact, par des polynômes trigonométriques; (b) Si une mesure $\mu$ bornée sur $G$ vérifie

$$
\int \phi(x) d \mu(x)=0
$$

pour toute $\phi$ élémentaire, elle est identiquement nulle.

Démonstration. (1) L'équivalence de (a) et (b) résulte immédiatement du Lemme 3.

(2) (a) étant vrai pour les fonctions de la forme $f * f^{\sim}(f \in L)$-qui sont de type positif-l'est aussi, d'après (I 7 ), pour $f * g^{\sim}$-donc pour toute $h \in L$ et finalement pour toute fonction continue.

16. Algèbre des spectres. Nous allons montrer dans cette section com- 
ment on peut, à l'aide des "spectres," introduire une topologie dans l'ensemble des représentations irréductibles de $G$. Le résultat, on le verra, n'est pas parfait: cela tient à ce que le Problème 1 n'est pas résolu.

Désignons par $G^{-}$l'ensemble des représentatins unitaires irréductibles de $G$, non identiquement nulles, et définies à un isomorphisme près (un élément de $G^{\frown}$ est donc une "classe" de représentations).

Dans $\mathcal{P}_{0}$ considérons la famille $\Phi$ des parties $A$ qui vérifient les propriétés :

(1) $A$ est convexe et faiblement fermée.

(2) Si $\phi \in A$, il en est de même de

$$
\psi(x)=\sum \alpha_{i} \bar{\alpha}_{j} \phi\left(s_{i}^{-1} x s_{j}\right) \quad \text { dès que } \psi(e) \leqq 1 .
$$

(On a vu au no. 4 le moyen de former la plus petite partie de ce genre contenant une $\phi$ donnée de $P_{0}$.)

La famille $\Phi$ possède trivialement les propriétés suivantes:

(1) $\{0\}$ et $\Phi_{0}$ sont dans $\Phi$.

(2) Toute intersection (finie ou non) de $A_{i} \in \Phi$ est une $A \in \Phi$.

Considérons d'autre part deux éléments $A_{1}, A_{2} \in \Phi$ : quel est le plus petit $A \in \Phi$ qui contient $A_{1}$ et $A_{2}$ ? On va voir que $A$ est l'ensemble des

$$
\phi=\phi_{1}+\phi_{2} \quad\left(\phi_{1} \in A_{1}, \phi_{2} \in A_{2}, \phi(e) \leqq 1\right) .
$$

Tout d'abord, il est évident que $A$ contient les $\phi$ de la forme (a): il reste donc à prouver que ces éléments constituent effectivement une famille appartenant à $\Phi$.

(1) Les $\phi=\phi_{1}+\phi_{2}$ forment un ensemble convexe $B$ : car si $\phi^{\prime}=\phi_{1}^{\prime}+\phi_{2}^{\prime}$, on a

$$
\alpha \phi+(1-\alpha) \phi^{\prime}=\left[\alpha \phi_{1}+(1-\alpha) \phi_{1}^{\prime}\right]+\left[\alpha \phi_{2}+(1-\alpha) \phi_{2}^{\prime}\right] .
$$

De plus, cet ensemble convexe est faiblement fermé. Il suffit pour cela de prouver qu'il est faiblement compact.

Or, $A_{1}$ et $A_{2}$ le sont-donc aussi l'espace produit $A_{1} \times A_{2}-$ donc aussi, dans $A_{1} \times A_{2}$, l'ensemble des éléments $\left[\phi_{1}, \phi_{2}\right]$ qui vérifient

$$
\phi_{1}(e)+\phi_{2}(e) \leqq 1 \text {. }
$$

La proposition résulte alors du fait que l'application $\left[\phi_{1}, \phi_{2}\right] \rightarrow \phi_{1}+\phi_{2}$ de $A_{1} \times A_{2}$ dans $L^{\infty}$ est continue pour les topologies envisagées.

(2) $\mathrm{Si} \phi \in B$, et si

$$
\psi(x)=\sum \alpha_{i} \bar{\alpha}_{j} \phi\left(s_{i}^{-1} x s_{j}\right) \quad \text { vérifie } \quad \psi(e) \leqq 1,
$$

on a $\psi \in B$. Car, si $\phi=\phi_{1}+\phi_{2}$, on a

$$
\psi(x)=\sum \alpha_{i} \bar{\alpha}_{j} \phi_{1}\left(s_{i}^{-1} x s_{j}\right)+\sum \alpha_{i} \bar{\alpha}_{j} \phi_{2}\left(s_{i}^{-1} x s_{j}\right)=\psi_{1}(x)+\psi_{2}(x)
$$

avec

$$
\psi_{1} \in A_{1}, \quad \psi_{2} \in A_{2}, \quad \psi_{1}(e) \leqq 1, \quad \psi_{2}(e) \leqq 1
$$


La proposition est donc démontrée.

Ceci étant, il est clair que toute $A \in \Phi$ est une partie régulière (on observera que, si $A_{1}$ et $A_{2}$ sont seulement régulières, il n'est pas vrai, en général, que la plus petite partie régulière contenant $A_{1}$ et $A_{2}$ soit l'ensemble des $\phi_{1}+\phi_{2} \in \mathcal{P}_{0}$, $\phi_{1} \in A_{1}, \phi_{2} \in A_{2}$; c'est pourquoi nous avons imposé la condition plus restrictive (2)). Par suite, tout point extrémal $\neq 0$ de $A$ est une $\phi$ élémentaire norméeet définit donc un élément de $G^{\widehat{ }}$. D'où la

Definition 4. On appellera spectre d'une $A \in \Phi$ l'ensemble $\sigma_{\Delta} \subset G^{\wedge}$ des représentations unitaires irréductibles définies par les points extrémaux de $A$. $L^{\prime}$ 'ensemble des parties $\sigma_{A}$ de $G^{-}$ainsi obtenues sera designé par $\mathfrak{F}$.

On a alors le

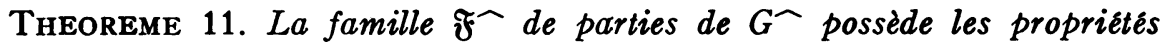
suivantes:

(1) $\mathcal{F}^{\wedge}$ contient $G^{-}$et la partie vide de $G^{\wedge}$ :

(2) Toute intersection d'elements de $\mathfrak{F}$ - est dans $\mathfrak{F}$.

(3) Toute réunion finie d'eléments de $\mathfrak{F}$ - est dans $\mathfrak{F}$.

Démonstration. (1) résulte du fait que $P_{0}$ et $\{0\}$ appartiennent à $\Phi$.

(2) résulte de la propriété analogue de $\Phi$.

(3) se démontre comme suit (nous faisons la démonstration dans le cas de 2 éléments): soient $\sigma_{A_{1}}$ et $\sigma_{A_{2}} \in F$. Soit $A$ le plus petit élément de $\Phi$ contenant $A_{1}$ et $A_{2}$. Il est clair que

$$
\sigma_{A} \supset \sigma_{A_{1}} \cup \sigma_{A_{2}} .
$$

Par ailleurs, si la fonction élémentaire normée $\phi$ est dans $A$, on a

$$
\phi=\phi_{1}+\phi_{2}, \quad \phi_{1} \in A_{1}, \quad \phi_{2} \in A_{2}:
$$

donc $\phi_{1}=\lambda_{1} \phi, \phi_{2}=\lambda_{2} \phi\left(0 \leqq \lambda_{1}, \lambda_{2} ; \lambda_{1}+\lambda_{2}=1\right)$; il en résulte que $\phi$ appartient au moins à l'un des deux ensembles $A_{1}, A_{2}$ : donc

$$
\sigma_{A} \subset \sigma_{A_{1}} \cup \sigma_{A_{2}}
$$

et finalement

$$
\sigma_{A}=\sigma_{A_{1}} \cup \sigma_{A_{2}} .
$$

L'importance du théorème précédent est que les propriétés (1), (2), (3) de $\mathfrak{F}$ permettent de considérer $\mathfrak{F}^{\wedge}$ comme étant la famille des ensembles fermés associée a une topologie convenable sur $G^{-}$. La première question que se pose relativement à cette topologie est celle de savoir si elle est séparée-c'est à dire si toute partie de $G^{\widehat{ح}}$ réduite à un seul point est dans $\mathfrak{F}^{\curlywedge}$. Or ceci revient à la question suivante: soit $\left\{\Re \in, U_{x}\right\}$ une structure unitaire irréductible sur $G$; dans $L^{\infty}$, soit $\Sigma$ l'ensemble des fonctions élémentaires normées associées à cette structure; c'est à dire de la forme 


$$
\phi(x)=\left(X, U_{x} X\right)
$$$$
(X \in \mathfrak{K}, \phi(e)=1)
$$

existe-t-il une partie $A \in \Phi$ dont $\Sigma$ constitue exactement l'ensemble des points extrémaux? En d'autres termes, on est ramené au problème 1.

Il en résulte qu'on peut affirmer que la topologie de $G^{\wedge}$ est séparée si les représentations irréductibles de $G$ sont de dimension finie: donc en particulier si $G$ est abélien ou compact. Il est facile de voir que, si $G$ est abélien, $G^{\wedge}$ est, en tant qu'espace topologique, homéomorphe au groupe dual de $G$. Dans le cas où $G$ est compact, les remarques faites précédemment prouvent que $G$. est discret.

Si $G$ est discret, et si $G^{\wedge}$ est séparé, on peut voir de la façon suivante que $G^{\wedge}$ est compact: soit $F \subset \mathfrak{F}$ une famille de fermés telle que toute intersection finie d'ensembles de $F$ soit non vide; soit $\Phi_{F} \subset \Phi$ l'ensemble des $A \in \Phi$ avec $\sigma_{A} \in F$. Appelons $S_{A}$ l'ensemble des $\phi \in A$ avec $\phi(e)=1: S_{A}$ est une partie fermée de l'espace compact constitué par les $\phi \in \mathcal{P}_{0}$ avec $\phi(e)=1$ (du fait que $G$ est discret, $\phi(e)$ est en effet une fonction faiblement continue sur $\mathcal{P}_{0}$ ).

D'après l'hypothèse faite sur $F$, les $S_{\boldsymbol{A}}$ ont donc au moins un point commun $\phi_{0}$, avec $\phi_{0}(e)=1$ : donc l'intersection des $A \in \Phi_{F}$ ne se réduit pas à zéro-ce qui prouve que, dans $G^{-}$, les $\sigma \in F$ ont une intersection non vide-et donc

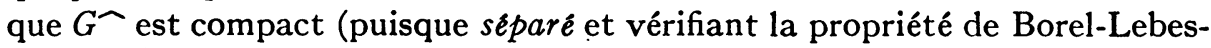
gue pour les fermés).

Il serait évidemment désirable que la topologie ainsi introduite dans $G^{-}$ fât étudiée à fond, au moins dans le cas, certainement plus simple, des groupes représentables dans les groupes compact. Il est en tout cas hors de doute que la topologie en question est beaucoup mieux adaptée à une éventuelle extension de la "dualité," que la topologie discrète introduite par Tannaka $\left.{ }^{26}\right)$ (topologie qui ne convient évidemment qu'aux groupes compacts).

17. Extension du théorème de $\mathrm{S}$. Bochner. Le classique théorème de S. Bochner peut aussi s'étendre à un groupe quelconque; mais ici encore, le résultat est imparfait-toujours à cause des problèmes 1, 2, 3! Au surplus, même quand ces problèmes sont résolus (ce qui est le cas des groupes compacts), le résultat présente, comme on le verra, une imperfection essentielle, qui tient au caractère non abélien des groupes envisagés.

Nous désignerons par $\Gamma^{-}$l'ensemble des fonctions élémentaires normées et de leurs limites faibles non identiquement nulles (ici intervient le problème no. 2). Muni de la topologie faible, $\Gamma^{\frown}$ est localement compact, puisque c'est le complémentaire du point $0 \in \mathscr{P}_{0}$ dans l'adhérence faible (espace compact) de l'ensemble des $\phi$ élémentaires normées; de plus, on voit que 0 joue, vis à vis de $\Gamma^{-}$, le rôle du point a l'infini ${\left({ }^{27}\right)}^{2}$. Désignons par $\Lambda_{\infty}$ l'ensemble des fonctions continues sur $\Gamma^{\wedge}$ que "s'annulent à l'infini" (cf. p. 5, la définition de

(æ) S. Tannaka, Dualität der nicht-Kommutativen Gruppen, Tohơku Math. J. t. 53 (1939).

(27) N. Bourbaki, Topologie Generale, chap. 1, pp. 65-67 (Théorème d'Alexandroff). 


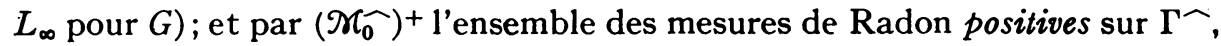
de norme $\leqq 1$. Rappelons (Lemme 1a) que $\left(\mathscr{X}_{0} \widehat{)}{ }^{+}\right.$est vaguement compact.

Pour toute $f \in E$, la fonction de

$$
(f, \phi)=\int f(x) \phi(x) \frac{d x}{(\rho(x))^{1 / 2}} \quad\left(|(f, \phi)| \leqq\|f\| ; \phi \in \Gamma^{\curlyvee}\right)
$$

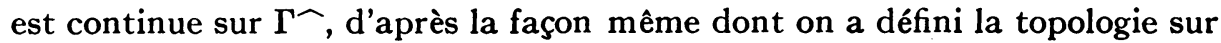

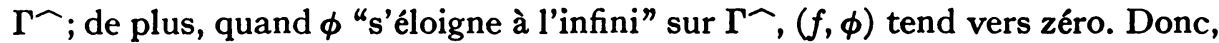
pour chaque $f \in E,(f, \phi)$ est dans $\Lambda_{\infty}$ (généralisation d'un théorème classique de Lebesgue). Si donc $\mu \widehat{\sim} \in\left(\mathcal{X}_{0} \widehat{)}\right)^{+}$, l'expression

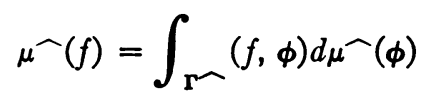

a un sens, et représente évidemment une fonctionnelle linéaire sur $E$, bornée: $|\mu \frown(f)| \leqq\|\mu-\|_{1} \cdot\|f\|$, et réelle (car $(f, \phi)$ est réelle et $\mu^{-}$positive).

Donc il éxiste un élément bien déterminé $\phi_{\mu}-\in E^{\prime}$ tel que

$$
\int f(x) \phi_{\mu} \frown(x) \frac{d x}{(\rho(x))^{1 / 2}} \equiv\left(f, \phi_{\mu} \uparrow\right)=\int(f, \phi) d \mu \smile(\phi)
$$

pour toute $f \in E$; de plus on a

$$
\left\|\phi_{\mu} \sim\right\|_{\infty} \leqq\|\mu-\|_{1}
$$

enfin, pour toute $g \in L$, on a $(g * \tilde{g}, \phi) \geqq 0$ pour $\phi \in \Gamma^{\widehat{ }}$, donc, puisque $\mu^{-}$ est positive,

$$
\left(g * g^{\sim}, \phi_{\mu} \sim\right) \geqq 0,
$$

ce qui prouve que $\phi_{\mu}-\in \mathcal{P}_{0}$.

Le théorème de Bochner consiste alors à prouver que les $\phi_{\mu}-\in \mathcal{P}_{0}$ ainsi obtenues constituent tout $\boldsymbol{P}_{0}$.

Or, si $\mu$ est formée de masses discrètes, on obtient pour $\phi_{\mu}$ - un polynôme trigonométrique à coefficients positifs, et réciproquement: nous savons donc que les $\phi_{\mu}$ - sont faiblement denses dans $\boldsymbol{P}_{0}$.

Il s'ensuit que le résultat sera atteint si l'on prouve que les $\phi_{\mu}$ - forment un ensemble faiblement ferme.

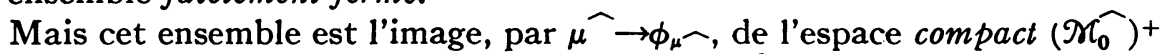
on est ainsi ramené à prouver que l'application $\mu \widehat{\mu} \rightarrow \phi_{\mu}$ - est continue pour les topologies envisagées (vague dans $\left(\mathcal{H}_{0}\right)^{+}$, faible dans $\left.E^{\prime}\right)$. Ce qui est immédiat: si $\mu \widehat{\widehat{T}} \in\left(\mathcal{X}_{0}\right)^{+}$converge vaguement vers $\mu_{0} \in\left(\mathcal{X}_{0}\right)^{+}$, pour toute fonction $l(\phi)$ de $\widehat{\Lambda_{\infty}}$ on aura

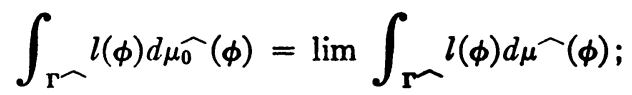


donc en particulier

$$
\begin{aligned}
& \left(f, \boldsymbol{\phi}_{\mu_{0}}\right) \equiv \int(f, \phi) d \mu_{\hat{0}}(\phi)=\lim \int(f, \phi) d \mu \widehat{\mu}(\phi) \\
& =\lim \left(f, \boldsymbol{\phi}_{\mu_{0}}\right)
\end{aligned}
$$

pour toute $f \in E$ : d'où la convergence faible de $\phi_{\mu}$ vers $\phi_{\mu} \widehat{0}$. Observons maintenant qu'on pourrait faire les mêmes raisonnements avec un partie régulière quelconque de $\boldsymbol{P}_{0}$. Alors nous obtenons la généralisation suivante du théorème de Bochner:

Theoreme 12. A toute fonction continue et de type positif $\phi(x)$ définie sur $G$ est associée au moins une mesure $\mu^{\widehat{\lambda}}$ défnie sur $\Gamma^{\widehat{\gamma}}$, positive et de norme $\| \widehat{\|}_{1}=\phi(e)$, telle qu'on ait pour toute $f \in L$ :

$$
\int_{G} f(x) \phi(x) \frac{d x}{(\rho(x))^{1 / 2}} \equiv(f, \phi)=\int_{\mathbf{r}}(f, x) d \mu \smile(\chi) .
$$

De plus, on peut supposer $\mu^{\widehat{T}}$ portée par l'adherence dans $\Gamma^{\widehat{d}}$ du spectre de $\phi$.

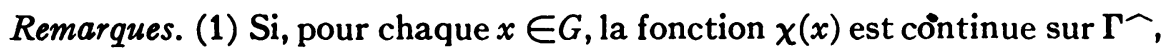
on peut écrire

$$
\phi(x)=\int_{\Gamma} \chi(x) d \mu-(x)
$$

c'est le cas si $G$ est abélien, ou compact; ou si $G$ est discret (ce qui est ici un résultat trivial).

En particulier, si $G$ est compact, les remarques faites plus haut sur la structure topologique de $\Gamma^{-}$permettent d'obtenir facilement la

PROPOSITION 9. Sur un groupe compact, toute fonction continue de type positif $\phi(x)$ est de la forme

$$
\phi(x)=\sum \lambda_{n} \phi_{n}(x)
$$

où les $\phi_{n}(x)$ sont des fonctions élémentaires normées et où les $\lambda_{n}$ sont $\geqq 0$ et verifient

$$
\phi(e)=\sum \lambda_{n}<+\infty .
$$

(2) Si $G$ n'est pas abélien, la mesure $\mu$ - n'est pas déterminée entièrement par $\phi$, comme le montre l'exemple simple suivant: soit $\left\{\mathfrak{H}, U_{x}\right\}$ une structure unitaire irréductible de dimension $n<+\infty$, et $\chi(x)$ le "caractère" de cette représentation. Alors à chaque base orthonormale $X_{1}, \cdots, X_{n}$ dans $\mathfrak{H}$ correspond une représentation 


$$
\chi(x)=\int_{\Gamma} \phi(x) d \mu \frown(\phi)
$$

où $\mu$ est formée de masses +1 placées aux points

$$
\phi_{i}(x)=\left(X_{i}, U_{x} X_{i}\right) \text { de } \Gamma^{\Upsilon} \text {. }
$$

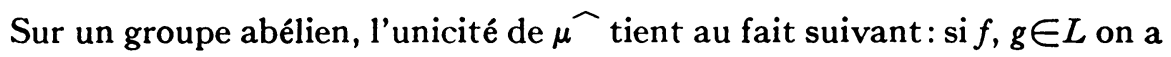

$$
(f * g, x)=(f, x) \cdot(g, x)
$$

et ceci d'après la propriété $\chi(x y)=\chi(x) \chi(y)$ des caractères. Il s'ensuit que les fonctions $(f, \chi)$ forment un sous-aneau de $\widehat{\Lambda_{\infty}}$; de plus, si $\chi_{1} \neq \chi_{2}$, il existe un $f$ avec $\left(f, \chi_{1}\right) \neq\left(f, \chi_{2}\right)$ :alors un théorème de Stone prouve que ce sous-anneau est dense dans $\Lambda_{\infty}^{-}$-d'où l'unicité de $\mu$.

18. Le spectre $d^{\prime}$ une fonction quelconque. Considérons l'espace de Banach $L^{1}$, et son dual $L^{\infty}$. Si $\phi \in L^{\infty}$, désignons par $V_{\phi}$ la plus petite variété linéaire faiblement fermée de $L^{\infty}$ qui contient les

$$
\phi_{s, t}(x)=\phi\left(s^{-1} x t\right) \text {. }
$$

Nous appellerons spectre de $\phi$ l'ensemble des fonctions élémentaires normées contenues dans $V_{\phi}$ (en supposant bien entendu $\left.\phi \not \equiv 0\right)$. Nous prouverons ailleurs que, sur un groupe abélien, toute $\phi \in L^{\infty}$ possède un spectre non vide (ce qui constitue l'extension d'un théorème récent de $\mathrm{A}$. Beurling $\left({ }^{28}\right)$ ). Notre intention est ici de prouver cette propriété, non pour toute $\phi \in L^{\infty}-$ ce qui parait assez compliqué-mais pour des catégories assez étendues de fonctions.

(1) Toute fonction de type positif possède un spectre non vide. (On notera que le mot spectre n'a pas exactement le même sens que dans les pages précédentes, où il a déja au moins deux significations distinctes; du moins, il n'est pas démontré que le sens présent coincide avec la définition 2.) Considérons $A=\mathscr{P}_{0} \cap V_{\phi}$ : c'est une partie régulière de $\mathscr{P}_{0}$. En effet, $A$ est trivialement convexe et faiblement fermée; de même, si $\psi \in A$ on a aussi $\psi / \psi(e) \in A$; enfin, si $\psi \in A$ et si $0 \ll \theta \ll \psi, \theta$ est limite, uniforme sur $G$, de $\sum \alpha_{i} \psi_{s_{i}} \in V_{\phi}$ donc $\theta \in V_{\phi} \cap \mathcal{P}_{0}$ - et $A$ est bien régulière. Il résulte de là que le spectre de $\phi$ n'est pas vide; et que ce spectre constitue une base $\left({ }^{29}\right.$ ) de $V_{\phi}$ (pour la topologie faible).

(2) Toute combinaison linéaire de fonctions de type positif possède un spectre non vide.

Soit en effet

$$
\phi=\phi_{1}-\phi_{2}+i\left(\phi_{3}-\phi_{4}\right) \quad \text { où les } \phi_{r} \in \mathcal{P} .
$$

(28) A. Beurling, Un theorème sur les fonctions uniformément continues . . , Acta Math. t. 77 (1945). R. Godement, Theorèmes tauberiens et théorie spectrale, Ann. Ecole Norm. (1947).

(29) C'est a dire que toute $\psi \in V_{\phi}$ est limite faible de fonctions de la forme $a_{1 \chi_{1}}+\cdots+a_{n} \chi_{n}$ où les $\chi_{i}$ dans le spectre de $\phi$.

On notera que, même si $G$ est abélien, cette dernière propriété ne vaut pas pour toute $\phi \in L^{\infty}$-mais seulement pour des $\phi$ particulières, ou des $G$ particuliers (par exemple comparts). 
Posons

$$
\psi=\phi_{1}+\phi_{2}+\phi_{3}+\phi_{4}
$$

comme $\psi$ majore (au sens de $\Phi$ ) les $\phi_{p}$, on peut écrire

$$
\phi_{p}(x)=\left(\xi_{p}, U_{x} \psi\right) \quad\left(\xi_{p} \in \mathfrak{F}_{\psi}\right)
$$

d'où

$$
\phi(x)=\left(\xi, U_{x} \psi\right)
$$

où $\xi$ est un élément bien déterminé de $\mathfrak{F C}_{\psi}$. Soit $\mathfrak{H}$ le sous-espace fermé de $\mathfrak{C}_{\psi}$ engendré par les $U_{x} \xi$; et soit $\eta$ la projection orthogonale de $\psi$ sur $\mathfrak{H C}$. On a

$$
\phi(x)=\left(\xi, U_{x} \eta\right)
$$

et comme $\eta$ est limite forte, dans $\mathfrak{H}$, de $\sum \alpha_{i} U_{s_{i}} \xi$, il en résulte que la fonction de type positif

$$
\theta(x)=\left(\eta, U_{x} \eta\right)
$$

est limite uniforme de $\sum \alpha_{i} \phi\left(s_{i}^{-1} x\right)$ : donc $V_{\phi}$ contient $\theta$. Mais alors $V_{\theta} \subset V_{\phi}$. Réciproquement, l'élément $\xi \in \mathfrak{F C}$ est limite forte de $\sum \beta_{j} U_{t_{i}} \eta$ : donc, par le même raisonnement, $V_{\phi} \subset V_{\theta}$. Finalement, $V_{\phi}=V_{\theta}$, et on est ramené au cas précédent.

(3) Toute fonction continue, de carré sommable pour $d x$, possède un spectre non vide. Car si $\phi$ est une telle fonction, $V_{\phi}$ contient la fonction

$$
\psi(x)=\phi * \phi^{\sim}(x)=\int \phi(x y) \overline{\phi(y)} d y
$$

qui est de type positif-et non nulle si $\phi$ ne l'est pas. Donc, d'après $V_{\psi} \subset V_{\phi}$, le spectre de $\phi$ contient au moins celui de $\psi$.

Ici encore, le spectre de $\phi$ constitue une base faible de $V_{\phi}$. En effet, pour toute $\theta \in L$, la fonction

$$
\phi * \theta^{\sim}(x)=\int \phi(x y) \overline{\theta(y)} d y
$$

est dans $V_{\phi}$-et, de plus, est combinaison linéaire de fonctions de type positif. Donc le spectre de $\phi * \theta^{\sim}$-contenu dans celui de $\phi$-permet d'approcher $\phi * \theta^{\sim}$; mais les $\phi * \theta^{\mathcal{L}}$ permettent aussi d'approcher les $\phi_{s, t}$ : le spectre de $\phi$ constitue donc bien une base de $V_{\phi}$.

Nous n'insisterons pas davantage ici sur ces questions. Comme on le verra pour le cas des groupes abéliens, l'existence du spectre repose sur les propriétés algébrico-topologiques de l'anneau normé $L^{1}$ : la première chose à faire pour obtenir des résultats complets sera donc une étude des anneaux normés non abeliens. 


\section{CHAPITRE III \\ LE SPECTRE PONCTUEL D'UNE FONCTION DE TYPE POSITIF}

19. Position du problème. D'après le théorème de Bochner, toute fonction continue de type positif sur le groupe additif $R^{1}$ des nombres réels est de la forme

$$
\phi(x)=\int_{-\infty}^{+\infty} e^{i \lambda x} d \mu(\lambda)
$$

où $\mu$ est une mesure de Radon positive et bornée. On peut décomposer $\mu$ en la somme de deux mesures positives: l'une $\mu_{p}$ formée de masses ponctuelles $\alpha_{n}>0$ placées en des points $\lambda_{n}$; l'autre $\mu_{c}$ est "continue" (c'est à dire telle que la masse d'un point soit toujours nulle). D'où

$$
\phi(x)=\sum \alpha_{n} e^{i \lambda_{n} x}+\int_{-\infty}^{+\infty} e^{i \lambda x} d \mu_{c}(\lambda)
$$

avec $\sum \alpha_{n}=\left\|\mu_{p}\right\|_{1}<+\infty$. Le $\sum$ représente donc une fonction presque periodique de type positif $\left({ }^{30}\right)$.

Le but de ce chapitre est d'éendre au cas d'un groupe quelconque cette décomposition. On le fera par des méthodes qui, 'bien entendu, sont tout à fait différentes de la précédente-et qui, espérons-nous, mettront mieux en lumière la nature de la décomposition envisagée. Nous rapellerons tout d'abord la notion - pas encore classique-de produit tensoriel de deux espaces de Hilbert.

20. Produit tensoriel de deux espaces de Hilbert(.11). Soient $\mathcal{F}, \mathcal{F C}^{\prime}$ deux espaces de Hilbert (éléments de $\mathfrak{H}: X, Y, \ldots$; éléments de $\mathfrak{H C}^{\prime}: X^{\prime}, Y^{\prime} ; \ldots$ ). Considérons leur produit direct $\mathfrak{H C} \mathfrak{H C}^{\prime}$ (ensemble des couples $\left[X, X^{\prime}\right], \cdots \cdots$ ) et l'espace vectoriel $\mathbb{R}$ des combinaisons linéaires formelles

$$
\sum_{i} \lambda_{i}\left[X_{i}, X_{i}^{\prime}\right]
$$

( $\lambda_{i}$ complexes).

Dans l'espace obtenu, on va définir un produit scalaire par la formule

$$
\left(\sum_{i=1}^{p} \lambda_{i}\left[X_{i}, X_{i}^{\prime}\right], \sum_{j=1}^{q} \mu_{j}\left[Y_{j}, Y_{j}^{\prime}\right]\right)=\sum_{i, j} \lambda_{i} \mu_{j}\left(X_{i}, Y_{j}\right) \cdot\left(X_{i}^{\prime}, Y_{j}^{\prime}\right) .
$$

La bilinéarité de cette expression est évidente; il reste à voir sa "positivité" -c'est à dire que l'on a

${ }^{\left({ }^{30}\right)}$ Pour plus de détails, cf. S. Bochner, Vorlesungen über Fouriersche Integrale (spécialement pp. 77-82).

(81) F. J. Murray and J. v. Neumann, On rings of operators, Ann. of Math. t. 37 (1936) pp. 116-229. 


$$
\sum \lambda_{i} \bar{\lambda}_{j}\left(X_{i}, X_{j}\right)\left(X_{i}^{\prime}, X_{i}^{\prime}\right) \geqq 0 .
$$

Démonstration. Soit $V$ la variété linéaire-de dimension $\leqq p$-soustendue dans $\mathcal{H C}$ par les $X_{i}$; soit $e_{\alpha}$ une base orthonormale de $V$; alors si

$$
\lambda_{i} X_{i}=\sum_{\alpha} \rho_{i}^{\alpha} e_{\alpha}
$$

on a

$$
\sum \lambda_{i} \bar{\lambda}_{j}\left(X_{i}, X_{j}\right)\left(X_{i}^{\prime}, X_{j}^{\prime}\right)=\sum_{\alpha}\left(\sum_{i} \rho_{i}^{\alpha} X_{i}^{\prime}, \sum_{i} \rho_{i}^{\alpha} X_{i}^{\prime}\right) \geqq 0 .
$$

Il est clair que les éléments de $\mathbb{R}$ dont la "norme" au sens précédent est nulle forment un sous-espace vectoriel NCR; et que le produit scalaire de deux éléments de $R$ ne dépend que des "classes d'équivalence modulo- $N^{\text {" }}$ auxquelles ils appartiennent; on peut donc considérer le produit scalaire comme défini sur l'espace quotient $R_{0}=R / N$, et alors est réalisée la condition: pour qu'un élément de $\mathbb{R}_{0}$ soit $n u l$, il faut et $i l$ suffit que sa norme le soit.

On désignera symboliquement par $X \wedge X^{\prime}$ l'image de l'élément $\left[X, X^{\prime}\right] \in R$ par l'application "canonique" de $R$ sur $R_{0}$. On voit facilement que

$$
(\lambda X) \wedge X^{\prime}=\lambda\left(X \wedge X^{\prime}\right)
$$

etc., et l'on a, par définition du produit scalaire:

$$
\left(X \wedge X^{\prime}, Y \wedge Y^{\prime}\right)=\left(X, X^{\prime}\right) \cdot\left(Y, Y^{\prime}\right) \text {. }
$$

De plus tout élément de $R_{0}$ est de la forme

$$
X_{1} \wedge X_{1}^{\prime}+\cdots+X_{n} \wedge X_{n}^{\prime} \quad\left(X_{i} \in \mathfrak{F}, X_{i}^{\prime} \in \mathfrak{H}^{\prime}\right)
$$

( $n$ pouvant bien entendu prendre toute valeur entière $>0$ ). Nous obtiendrons alors le produit tensoriel $\mathfrak{F C} \wedge \mathcal{F C}^{\prime}$ en complètant $\mathbb{R}_{0}$ pour la norme introduite ci dessus, comme on l'a fait déja au chap. 1 pour définir $\mathfrak{K C}_{\phi}$.

Cela revient évidemment à considérer les sommes infinies

$$
\sum_{1}^{\infty} X_{i} \wedge X_{i}^{\prime}
$$

vérifiant la condition de convergence

$$
\sum_{i, j}\left|\left(X_{i}, X_{j}\right) \cdot\left(X_{i}^{\prime}, X_{j}^{\prime}\right)\right|<+\infty .
$$

21. Produit de deux fonctions de type positif. Soient $\phi$ et $\phi^{\prime}$ deux fonctions continues de type positif; on a donc

$$
\begin{aligned}
\phi(x) & =\left(\phi, U_{x} \phi\right) & \text { dans } & \mathfrak{H}_{\phi}, \\
\phi^{\prime}(x) & =\left(\phi^{\prime}, U_{x} \phi^{\prime}\right) & \text { dans } & \mathfrak{F C}_{\phi^{\prime}} .
\end{aligned}
$$

Dans le produit tensoriel $\mathfrak{H}_{\phi} \wedge \mathfrak{K}_{\phi^{\prime}}$, on peut naturellement définir une repré- 
sentation unitaire continue de $G$ en posant

$$
U_{x} \sum \xi_{i} \wedge \xi_{i}^{\prime}=\sum U_{x} \xi_{i} \wedge U_{x} \xi_{i}^{\prime}
$$

et en étendant à tout $\mathfrak{H}_{\phi} \wedge \mathfrak{H}_{\phi^{\prime}}$ l'opérateur ainsi défini (ce qui est possible, puisqu'on a immédiatement

$$
\left.\left|U_{x} \sum \xi_{i} \wedge \xi_{i}^{\prime}\right|=\left|\sum \xi_{i} \wedge \xi_{i}^{\prime}\right|\right) .
$$

On obtient donc une structure unitaire qu'on notera

$$
\left\{\mathfrak{F}_{\phi}, U_{x}\right\} \wedge\left\{\mathfrak{F}_{\phi^{\prime}}, U_{x}\right\}
$$

et qui, dans le cas où $\mathfrak{K}_{\phi}$ et $\mathfrak{H}_{\phi^{\prime}}$, sont de dimensions finies, se réduit au "produit kroneckerien" des représentations données (cf. Weil, pp. 62-63).

Ceci étant, on a la propriété suivante, analogue au Théorème 4:

ThEOREME 13. Si $\phi$ et $\phi^{\prime}$ sont de type positif, il en est de même de leur produit $f=\phi \cdot \phi^{\prime} ;$ la structure unitaire $\left\{\mathcal{F}_{f}, U_{x}\right\}$ est contenue dans le produit tensoriel $\left\{\mathcal{F}_{\phi}, U_{x}\right\} \wedge\left\{\mathcal{F}_{\phi^{\prime}}, U_{x}\right\} ;$ les sommes $\sum \phi_{i}(x) \phi_{i}^{\prime}(x)$ (où $\left.\phi_{i} \in H_{\phi^{\prime}}, \phi_{i}^{\prime} \in H_{\phi^{\prime}}\right) a p-$ partiennent a $H_{f}$, et $y$ sont partout denses au sens de $C^{\infty}$; enfin la norme de $\phi_{i} \phi_{i}^{\prime}$ dans $\mathfrak{H}_{f}$ est au plus égale au produit des normes de $\phi_{i}\left(\right.$ dans $\left.\mathfrak{H}_{\phi}\right)$ et de $\phi_{i}^{\prime}$ (dans $\left.\mathcal{H C}_{\phi^{\prime}}\right)$.

Démonstration. (1) On a

$$
f(x)=\phi(x) \phi^{\prime}(x)=\left(\phi, U_{x} \phi\right) \cdot\left(\phi^{\prime}, U_{x} \phi^{\prime}\right)=\left(\phi \wedge \phi^{\prime}, U_{x} \phi \wedge \phi^{\prime}\right)
$$

d'où résulte que $f \in \mathcal{P}$ et que $\left\{\mathcal{H}_{f}, U_{x}\right\}$ est contenue dans $\left\{\mathcal{H}_{\phi}, U_{x}\right\} \wedge\left\{\mathcal{H}_{\phi^{\prime}}, U_{x}\right\}$; de manière précise, $\mathfrak{H}_{f}$ est isomorphe au sous espace vectoriel fermé $\mathfrak{H} \subset \mathfrak{H}_{\phi}$ $\wedge \mathfrak{K}_{\phi^{\prime}}$ soustendu par les $U_{x} \phi \wedge \phi^{\prime}$.

(2) Si les $\phi_{i}$ et $\phi_{i}^{\prime}$ sont dans $H_{\phi}$ et $H_{\phi^{\prime}}$ respectivement, on a

$$
\begin{aligned}
\phi_{i}(x) & =\left(\xi_{i}, U_{x} \phi\right) & \left(\xi_{i} \in \mathcal{F}_{\phi}\right), \\
\phi_{i}^{\prime}(x) & =\left(\xi_{i}^{\prime}, U_{x} \phi^{\prime}\right) & \left(\xi_{i}^{\prime} \in \mathcal{F}_{\phi^{\prime}}\right)
\end{aligned}
$$

d'où

$$
\begin{aligned}
\sum \phi_{i}(x) \phi_{i}^{\prime}(x) & =\sum\left(\xi_{i}, U_{x} \phi\right) \cdot\left(\xi_{i}^{\prime}, U_{x} \phi^{\prime}\right) \\
& =\left(\sum \xi_{i} \wedge \xi_{i}^{\prime}, U_{x} \phi \wedge \phi^{\prime}\right)
\end{aligned}
$$

$\operatorname{donc} \sum \phi_{i} \phi_{i}^{\prime} \in H_{f}$.

(3) Toute $\psi \in H_{f}$ étant d'après (1) de la forme

$$
\psi(x)=\left(X, U_{x} \phi \wedge \phi^{\prime}\right) \text { où } X \in \mathfrak{H C} \subset \mathfrak{H C}_{\phi} \wedge \mathfrak{F}_{\phi^{\prime}},
$$

et $X$ étant, dans $\mathfrak{H}_{\phi} \wedge \mathcal{H}_{\phi^{\prime}}$, limite forte de $\sum \xi_{i} \wedge \xi_{i}^{\prime}$, on voit que $\psi$ est limite, uniforme $\operatorname{sur} G$, de fonctions

$$
\sum \phi_{i}(x) \phi_{i}^{\prime}(x) \quad\left(\phi_{i} \in H_{\phi}, \phi_{i}^{\prime} \in H_{\phi^{\prime}}\right) .
$$


(4) Si $\phi_{i} \in H_{\phi}$ et $\phi_{i}^{\prime} \in H_{\phi^{\prime}}$, l'image dans $\mathfrak{F}_{f}$ de l'élément $\phi_{i} \phi_{i}^{\prime} \in H_{f}$ est l'élément $X \in \mathcal{H}$ tel que

$$
\phi_{i}(x) \phi_{i}^{\prime}(x)=\left(X, U_{x} \phi \wedge \phi^{\prime}\right):
$$

c'est donc la projection sur $\mathfrak{H C}$ de l'élément $\xi_{i} \wedge \xi_{i}^{\prime} \in \mathfrak{K}_{\phi} \wedge \mathfrak{H}_{\phi^{\prime}}$ (on conserve les mêmes notations que plus haut). D'où résulte que sa norme est majorée par

$$
\left|\xi_{i} \wedge \xi_{i}^{\prime}\right|=\left|\xi_{i}\right| \cdot\left|\xi_{i}^{\prime}\right| .
$$

CQFD.

On reviendra plus loin sur ce théorème.

22. Topologies sur $\mathfrak{K}_{\phi}$. Soit $\phi \in \mathcal{P}$. On peut d'finir diverses topologies sur $\mathfrak{H}_{\phi}$; les plus intéressantes ici sont:

(1) La topologie $\mathfrak{H C}$-faible: $\xi \in \mathfrak{H}_{\phi}$ converge vers $\xi_{0}$ si, pour tout $\zeta \in \mathcal{K}_{\phi},(\xi, \zeta)$ converge vers $\left(\xi_{0}, \zeta\right)$.

(2) La topologie de la convergence simple: $\xi \in \mathfrak{C}_{\phi}$ converge vers $\xi_{0}$ si, pour tout $x \in G,\left(\xi, U_{x} \phi\right)$ converge vers $\left(\xi_{0}, U_{x} \phi\right)$.

(3) La topologie $C$-faible: $\xi \in \mathfrak{H}_{\phi}$ converge vers $\xi_{0}$ si l'élément de l'espace normé complet $C^{\infty}$ défini par la fonction $\left(\xi, U_{x} \phi\right)$ converge faiblement( $\left.{ }^{22}\right)$ vers l'élément correspondant associé à $\xi_{0}$.

Proposition 10. Sur toute partie de $\mathfrak{H}_{\phi}$, bornée au sens de la norme hilbertienne de $\mathfrak{H}_{\phi}$, les trois topologies précédentes coincident.

Démonstration. Soit $S$ la boule unité de $\mathfrak{F C}_{\phi}$ (au sens de la norme $\left.|\xi|=\left((\xi, \xi)_{\phi}\right)^{1 / 2}\right)$. On reste sur $S$.

(a) Que les topologie $\mathcal{H}$-faible et de la convergence simple coincident résulte de ce que les $U_{x} \phi$ soustendent tout $\mathfrak{H}_{\phi}$ et d'une propriété classique des topologies faibles hilbertiennes.

(b) Soit $\mu(f)$ une fonctionnelle linéaire bornée sur $C^{\infty}$. Désignons par la même lettre un élément $\xi \in \mathfrak{H C}_{\phi}$ et son image dans $H_{\phi}$ (c'est la fonction

$$
\left.\xi(x)=\left(\xi, U_{x} \phi\right)\right) .
$$

On a

donc

$$
\|\xi\|_{\infty} \leqq(\phi(e))^{1 / 2} \cdot|\xi|
$$

$$
|\mu(\xi)| \leqq K \cdot|\xi|
$$

et par suite

$$
\mu(\xi)=\left(\xi, \zeta_{\mu}\right)
$$

où $\zeta_{\mu} \in \mathfrak{H}_{\phi}$ : donc la convergence $\mathfrak{H}$-faible entraine la convergence $C$-faible.

(c) La réciproque est vraie aussi: il suffit d'observer que, pour chaque $x \in G$, la fonctionnelle linéaire

${ }^{\left({ }^{2}\right)} f \in C^{\infty}$ converge faiblement vers $f_{0}$ si, pour toute fonctionnelle linéaire $\mu(f)$ définie sur $C^{\infty}$, et continue au sens de la norme de $C^{\infty}, \mu(f)$ tend vers $\mu\left(f_{0}\right)$. Cette topologie est donc a distinguer de la topologie faible de $L^{\infty}$-et est du reste beaucoup plus compliquee. 


$$
\boldsymbol{\epsilon}_{x}(\xi)=\xi(x)=\left(\xi, U_{x} \phi\right)
$$

est bornée sur $C^{\infty}$, en sorte que la convergence $C$-faible entraine la convergence simple. CQFD.

Remarque. Soit $K$ une partie compacte de $G$; l'application $x \rightarrow U_{x} \phi$ étant fortement continue, les $U_{x} \phi(x \in K)$ constituent une partie fortement compacte de $\mathcal{H}_{\phi}$. En appliquant le théorème de Gelfand déja cité( $\left({ }^{17}\right)$, on voit que, sur une partie bornée de $\mathcal{F}_{\phi}$, la convergence faible de $\xi$ vers $\xi_{0}$ équivaut encore à la convergence, uniforme sur tout compact, de $\xi(x)$ vers $\xi_{0}(x)$.

23. Ergodicité des fonctions de type positif. Soit $\phi$ une fonction continue et bornée sur $G: \phi \in C^{\infty}$. Notons $K_{\phi}^{o}$ l'ensemble des fonctions de la forme

$$
\psi(x)=\sum \alpha_{i} \phi\left(s_{i}^{-1} x\right) \quad\left(\alpha_{i} \geqq 0, \sum \alpha_{i}=1\right)
$$

et de leurs limites uniformes $\operatorname{sur} G: K_{\phi}^{o}$ est la plus petite partie convexe fermée de $C^{\infty}$ contenant les translatées a gauche de $\phi$. Soit $K_{\phi}^{d}$ le convexe analogue relatif à la translation droite.

DÉfInITION. Une $\phi$ est ergodique à gauche (à droite) si $K_{\phi}^{o}\left(K_{\phi}^{d}\right)$ contient une fonction constante sur $G ; \phi$ est ergodique si elle l'est à gauche et d droite.

On sait( $\left.{ }^{23}\right)$ que, si $\phi$ est ergodique, $K_{\phi}^{q}$ et $K_{\phi}^{d}$ contiennent une seule et même constante; car si les constantes $M^{0}$ et $M^{d}$ sont dans $K_{\phi}^{o}$ et $K_{\phi}^{d}$ respectivement, on peut faire

$$
\left|\sum \alpha_{i} \phi\left(s_{i}^{-1} x\right)-M^{o}\right|<\epsilon, \quad\left|\sum \beta_{j} \phi\left(x t_{j}\right)-M^{d}\right|<\epsilon
$$

quel que soit $x \in G$-d'où $\left(\alpha_{i}>0, \sum \alpha_{i}=1 ; \beta_{j}>0, \sum \beta_{j}=1\right)$ :

$$
\left|\sum \alpha_{i} \beta_{j} \phi\left(s_{i}^{-1} t_{j}\right)-M^{0}\right|<\epsilon, \quad\left|\sum \alpha_{i} \beta_{j} \phi\left(s_{i}^{-1} t_{j}\right)-M^{d}\right|<\epsilon
$$

et par suite $M^{d}=M^{0}$-nombre bien déterminé qu'on notera

$$
\underset{t}{M}[\phi(t)] \quad \text { ou simplement } M(\phi) \text {. }
$$

On va alors prouver le

Theoreme 14. Toute combinaison linéaire de fonctions de type positif est ergodique; si au surplus $\phi \in \Phi$, on a

$$
M(\phi)=\inf _{\alpha_{i} \geqq 0, \sum \alpha_{i}=1} \sum \alpha_{i} \alpha_{j} \phi\left(s_{i}^{-1} s_{j}\right) \geqq 0 .
$$

Demonstration. (1) Rappelons d'abord le théorème suivant:

Lemme(34). Soient HC un espace de Hilbert et $G$ un groupe d'opérateurs uni445.

(23) J. v. Neumann, Almost periodic functions in a group, Trans. Amer. Math. Soc. t. 36, p.

(34) Ce lemme est un cas particulier d'un théorème de G. Birkhoff (Proc. Nat. Acad. Sci. U.S.A. t. 25 (1939) p. 625), et contient lui-mème le célèbre théorème ergodique de J. v. Neumann. 
taires dans $\mathfrak{H C}$; soit F le sous-espace vectoriel fermé de $\mathfrak{H C}$ constitué des $X \in \mathfrak{H C}$ tels que

$$
U X=X
$$

pour tout $U \in G$.

Alors, si $X \in \mathcal{H}$, le plus petit convexe fermé $K_{X}$ contenant les $U X(U \in G)$ rencontre $F$ en un point $X_{0}$ unique, qui est en même temps:

(1) la projection orthogonale de $X$ sur $F$;

(2) le point de $K_{X}$ dont la norme est minimum.

Démonstration du lemme. (a) Soit $X_{0}$ le point (unique d'après la convexité uniforme de 3 ) qui réalise le minimum de $|Y|$ sur $K_{X} . K_{X}$ étant trivialement invariant par $G$, et $U \in G$ tel que $\left|U X_{0}\right|=\left|X_{0}\right|$, on a $U X_{0}=X_{0}$ pour tout $U \in G$; donc $K_{X}$ rencontre $F$ en $X_{0}$.

Si $X$ est dans la variété $N=\nVdash \operatorname{co} F$, on a aussi $K_{X} \subset N$, donc $X_{0} \in N \cap F$, donc $X_{0}=0$.

(b) Soit $X_{0}^{\prime}$ la projection orthogonale de $X$ sur $F$; on a $X-X_{0}^{\prime} \in N$, d'où possibilité de faire, d'après la remarque précédente:

$$
\left|\sum \alpha_{i} U_{i}\left(X-X_{0}^{\prime}\right)\right| \equiv\left|\sum \alpha_{i} U_{i} X-X_{0}^{\prime}\right|<\epsilon \quad\left(\alpha_{i}>0, \sum \alpha_{i}=1\right)
$$

et par suite $X_{0}^{\prime} \in K_{X}$. De plus on a

$$
\begin{aligned}
\left|\sum \alpha_{i} U_{i} X\right|^{2} & =\left|\sum \alpha_{i} U_{i}\left(X-X_{0}^{\prime}\right)+X_{0}^{\prime}\right|^{2} \\
& =\left|\sum \alpha_{i} U_{i}\left(X-X_{0}^{\prime}\right)\right|^{2}+\left|X_{0}^{\prime}\right|^{2} \geqq\left|X_{0}^{\prime}\right|^{2}
\end{aligned}
$$

donc: $X_{0}^{\prime}$ est de norme minimum dans $K_{X}$, et par suite on a $X_{0}=X_{0}^{\prime}$.

(c) Soit enfin $X_{0}^{\prime \prime} \in K_{X} \cap F$. Alors $X_{0}^{\prime \prime}-X_{0} \in K_{X-X_{0}}$ et $\in F$ donc (puisque $\left.X-X_{0} \in N\right) \quad X_{0}^{\prime \prime}-X \in N \cap F$, et finalement $X_{0}^{\prime \prime}=X_{0}$ est le seul "point fixe" de $K_{X}$. CQFD.

(2) Ceci étant, soit $\phi$ une combinaison linéaire de fonctions de type positif : on a

$$
\phi(x)=\left(X, U_{x} Y\right)
$$

où $U_{x}$ est une représentation unitaire de $G$ dans un $\mathfrak{T C}$ convenable. Tout élément $\psi \in K_{\phi}^{\circ}$ est limite de fonctions de la forme

$$
\sum \alpha_{i} \phi\left(s_{i}^{-1} x\right)=\left(\sum \alpha_{i} U_{s_{i}} X, U_{x} Y\right)=\left(Z, U_{x} Y\right)
$$

où $Z \in K_{X}$, plus petit convexe fermé de $\mathfrak{F}$ contenant les $U_{x} X$. Soit $F$ l'ensemble des éléments de $3 C$ invariants par les $U_{x}$; si $X_{0}$ est la projection de $X$ sur $F$, il résulte du lemme précédent que $K_{\phi}^{o}$ contient la fonction

$$
\left(X_{0}, U_{x} Y\right)=\left(U_{x^{-1}} X_{0}, Y\right)=\left(X_{0}, Y\right)
$$

c'est à dire une constante. De même, tout $\theta \in K_{\phi}^{d}$ est limite de fonctions de la forme $\sum \beta_{j} \phi\left(x t_{j}\right)=\left(X, U_{x} \sum \bar{\beta}_{j} U_{t_{j}} Y\right)$; si donc $Y_{0}$ est la projection de $Y$ sur $F, K_{\phi}^{d}$ contient la fonction 


$$
\left(X, U_{x} Y_{0}\right)=\left(X, Y_{0}\right)
$$

donc une constante: d'où l'ergodicité de $\phi$. Bien entendu on a

$$
\left(X_{0}, Y\right)=\left(X, Y_{0}\right)=\left(X_{0}, Y_{0}\right)
$$

(tout projecteur est hermitien et identique à son carré).

(3) Si en outre $\phi$ est de type positif, on a

$$
\phi(x)=\left(X, U_{x} X\right)
$$

et

$$
\begin{aligned}
M(\phi)=\left(X_{0}, X_{0}\right)=\inf _{Y \in K_{x}}|Y|^{2} & =\inf _{\alpha_{i} \geqq 0, \sum \alpha_{i=1}}\left(\sum \alpha_{i} U_{s_{i}} X, \sum \alpha_{i} U_{s_{i}} X\right) \\
& =\inf _{\alpha_{i} \geqq 0, \sum \alpha_{i}=1} \sum \alpha_{i} \alpha_{j} \phi\left(s_{i}^{-1} s_{j}\right) \geqq 0 . \quad \text { CQFD. }
\end{aligned}
$$

Il est clair que la fonctionnelle $M(\phi)$ définie sur l'enveloppe linéaire $V$ de $P$ vérifie les propriétés suivantes:

$$
\begin{aligned}
M(\lambda \phi+\mu \psi) & =\lambda M(\phi)+\mu M(\psi), \\
|M(\phi)| & \leqq\|\phi\|_{\infty}, \\
\underset{x}{M}[\phi(s x t)] & =\underset{x}{M}[\phi(x)] \quad \text { quels que soient } s, t \in G ;
\end{aligned}
$$

c'est donc une fonctionnelle linéaire bornée sur le sous-espace $V$ de $C^{\infty}$, invariante par les translations ddroite et a gauche de G. De plus, on a

$$
M(\phi) \geqq 0 \quad \text { si } \phi(x) \geqq 0
$$
pour tout $x$, ou si $\phi \in P$.

24. Opérateurs spectraux. Le produit de deux fonctions de $\boldsymbol{P}$ étant dans $P$ (Théorème 13), la même propriété vaut pour $U$. Si donc $\phi, \psi \in V$, on peut définir la fonction

$$
\psi \times \phi(x)=M_{t}\left[\psi(t) \phi\left(t^{-1} x\right)\right]=M_{t}\left[\psi\left(x t^{-1}\right) \phi(t)\right] .
$$

L'opération $X$ possède évidemment les mêmes propriétés formelles que le produit de composition *. On verra plus loin que $\psi \times \phi \phi$ est une fonction presque-périodique de $x$.

Soit $\phi$ une fonction de type positif. En désignant par la même lettre un élément de $\mathfrak{H}_{\phi}$ et la fonction qui lui correspond dans $\mathfrak{H}_{\phi}$, on a, si $\psi \in \mathfrak{H}_{\phi}$ :

$$
\psi \times \phi(x)=M_{t}\left[\psi(t) \cdot\left(U_{t} \phi, U_{x} \phi\right)\right] .
$$

Des raisonnements identiques à ceux dont on s'est servi au chap. 1 pour définir les opérateurs de Radon prouvent alors qu'on a

$$
\psi \times \phi(x)=\left(\psi^{\prime}, U_{x} \phi\right)
$$

où $\psi^{\prime}$ est un élément de $\mathfrak{H}_{\phi}$ bien déterminé par $\psi$. Il est clair que l'application 
$\psi \rightarrow \psi \times \phi=\psi^{\prime}$ ainsi obtenue dans $\mathfrak{H C}_{\phi}$ est linéaire; elle est en outre permutable aux $U_{x}$, car on a

$$
\left(U_{s} \psi^{\prime}, U_{x} \phi\right)=\left(\psi^{\prime}, U_{s^{-1}} \phi\right)=\psi \times \phi\left(s^{-1} x\right)=\psi_{s} \times \phi(x) ;
$$

elle constitue aussi un opérateur hermitien positif borné dans $\mathfrak{H}_{\phi}$, car si $\psi$ est de la forme

$$
\psi=\sum \alpha_{i} U_{S_{i} \phi_{s_{i}}}
$$

on a

$$
\left(\psi^{\prime}, \psi\right)=\left(\psi^{\prime}, \sum \alpha_{i} U_{s_{i}} \phi\right)=\sum \bar{\alpha}_{i} \psi \times \phi\left(s_{i}\right)=\sum \bar{\alpha}_{i} \cdot M_{t}\left[\psi(t) \cdot\left(U_{t} \phi, U_{s_{i}} \phi\right)\right]
$$

et donc

$$
\left(\psi^{\prime}, \psi\right)=\underset{t}{M}[\psi(t) \cdot \overline{\psi(t)}]
$$

d'où

$$
0 \leqq\left(\psi^{\prime}, \psi\right) \leqq|\psi|^{2} \cdot \phi(e),
$$

et comme ces $\psi$ sont denses dans $\mathfrak{H C}_{\phi}$, la propriété s'étend à tout l'espace; enfin, et c'est ici le point essentiel, l'opérateur $\psi \rightarrow \psi^{\prime}=A \psi$ est complètement continu.

Pour cela, il suffit de prouver que, si $\psi$ converge $\mathcal{H}$-faiblement vers 0 dans $\mathfrak{H}_{\phi}$, en restant dans une boule de $\mathfrak{H}_{\phi}$, alors $A \psi$ converge fortement vers 0 dans $\mathfrak{H}_{\phi}$; or, $B$ désignant la racine carrée positive dè $A$, on a

$$
|A \psi|^{2}=(A \psi, A \psi)=(A B \psi, B \psi)=M_{t}\left[|B \psi(t)|^{2}\right]
$$

d'après $(\alpha)$, qui s'écrit de façon générale

$$
(A \psi, \psi)=M_{t}\left[|\psi(t)|^{2}\right]
$$

On est donc ramené à prouver que $M\left(|B \psi|^{2}\right)$ tend vers zéro. Mais, $\theta(t)=B \psi(t)$ converge $\mathfrak{H}$-faiblement vers zéro dans $\mathfrak{H C}_{\phi}$ puisque $B$ est borné, et reste dans une boule de $\mathfrak{K}_{\phi}$; il en est de même de $\bar{\theta}(t)$ qui appartient à $\mathfrak{K}_{\bar{\phi}}$, y converge $\mathfrak{K}_{\phi}$-faiblement vers zéro et y reste dans une boule; enfin, $\theta \bar{\theta}$ est dans une boule de $\mathcal{F}_{\phi \bar{\phi}}$ (Théorème 13), et $y$ converge simplement vers zéro (puisque $\theta(t) \rightarrow 0$ pour chaque $t$ ); d'après la proposition 10, $\theta \bar{\theta}$ tend donc $C$-faiblement vers zéro: comme $M(\phi)$ est une fonctionnelle linéaire bornée sur $C^{\infty}$, on a prouvé finalement que

$$
|A \psi|^{2}=M(\theta \bar{\theta}) \rightarrow 0
$$

et donc que $A$ est complètement continu.

Ce fait va nous permettre d'obtenir les résultats annoncés dans l'introduction de ce chapitre; et tout d'abord le 
Theoreme 15. Si $\phi, \psi$ sont combinaisons linéaires de fonctions de type positif, la fonction $\phi \times \psi(x)$ est presque périodique sur $G$.

Démonstration. (1) L'identité suivante, analogue à (I 7):

$$
4 \phi \times \psi^{\sim}=(\phi+\psi) \times(\phi+\psi)^{\sim}-(\phi-\psi) \times(\phi-\psi)^{\sim}+\cdots
$$

permet de se ramener au cas où $\phi=\psi$.

(2) Si $\psi \in \mathcal{U}$, il existe $\phi \in \mathcal{P}$ avec $\psi \in H_{\phi}$, c'est à dire

$$
\psi(x)=\left(\psi, U_{x} \phi\right)
$$

dans $\mathfrak{H}_{\phi}$. Comme $\psi$ est limite, dans $C^{\infty}$ (et même dans $\mathfrak{H}_{\phi}$ ) de combinaisons linéaires $\sum \alpha_{i} U_{s_{i}} \phi, \psi \times \psi$ est de même limite uniforme de combinaisons línéaires de translatées de $\phi \times \phi$ : on est donc ramené à prouver que $\phi \times \phi$ est presque périodique.

(3) Soit $\psi=\phi \times \phi$. On a $\psi_{s}=\phi_{s} \times \phi=A_{\phi s}$ ( $A$ défini ci-dessus pour $\mathfrak{F}_{\phi}$ ). Or $A$ est completement continu, et les $\phi_{s}$ sont dans une boule de $\mathfrak{H}_{\phi}-{ }^{\prime}$ 'est à dire dans un ensemble $\mathcal{H}$-faiblement compact: les $A_{\phi s}$ sont donc dans un ensemble fortement compact de $\mathfrak{H C}_{\phi}$-et à fortiori de $C^{\infty}$. CQFD.

Revenons maintenant à l'opérateur $A$ dans $\mathfrak{H}_{\phi}$. Etant complètement continu, hermitien positif, et permutable aux $U_{x}$, il est de la forme

$$
A=\sum_{i} \lambda_{i} E_{i} \quad\left(\lambda_{i} \geqq 0, \sum \lambda_{i}<+\infty\right)
$$

où les $E_{i}$ sont des projecteurs permutables aux $U_{x}$ et de dimension finie, associés à des variétés $V_{i} \subset \mathfrak{F}_{\phi}$. L'ensemble des $A \psi\left(\psi \in \mathfrak{F}_{\phi}\right)$ et de leurs limites constitue un sous-espace invariant fermé $\mathcal{H C}_{\phi}^{\text {p }}$, qui n'est autre que la somme directe des $V_{i}$; quant à la variété supplémentaire

$$
\mathfrak{H C}_{\phi}^{c}=\mathfrak{K}_{\phi} \ominus \mathfrak{K}_{\phi}^{p},
$$

ses éléments sont caractérisés par $A \psi=0$, c'est à dire par

$$
(A \psi, \psi) \equiv M_{t}\left[|\psi(t)|^{2}\right]=0
$$

En ce qui concerne les $\psi \in \mathcal{F}_{\phi}^{p}$, les fonctions de $H_{\phi}$ qui leur sont associées sont presque-périodiques-car elles sont limites uniformes de fonctions de la forme

$$
\left(A \theta, U_{x} \phi\right)=\theta \times \phi(x)
$$

elles-mêmes presque-périodiques (Théorème 15); de plus, ces $\psi \in \mathcal{C}_{\phi}^{p}$ sont les seuls éléments de $\mathfrak{H}_{\phi}$ associés à des fonctions presque périodiques: car si un $\psi \in \mathcal{F}_{\phi}^{c}$ vérifiait cette propriété, d'après $M_{t}\left[|\psi(t)|^{2}\right]=0$ on aurait, d'après une propriété bien connue des fonctions presque-périodiques, $\psi(t) \equiv 0$ donc $\psi=0$. Si alors on décompose $\phi$ en $\phi^{p}+\phi^{c}\left(\phi^{p} \in \mathcal{F}_{\phi}^{p}, \phi^{c} \in \mathcal{F}_{\phi}^{c}\right)$, on a la décomposition cherchée de $\phi(x)$ en la sommes de deux fonctions de type positif : l'une presque 
périodique, l'autre, au contraire, vérifiant $M\left(\left|\phi^{0}\right|^{2}\right)=0$.

Observons enfin que, si $M\left(|\phi|^{2}\right)=0, \mathfrak{B C}_{\phi}$ ne possède certainement aucun sous-espace invariant de dimension finie (sinon les fonctions associées à ce sous-espace seraient trivialement presque-périodiques; or $M\left(|\phi|^{2}\right)=0$ $=(A \phi, \phi)$ entraine $A \phi=0$ d'où

$$
A U_{\diamond} \phi=U_{\diamond} A \phi=0
$$

d'où $A=0$, et donc $M\left(|\psi|^{2}\right)=0$ pour toute $\left.\psi \in H_{\phi}\right)$. Et que cette condition, nécessaire pour que $M\left(\left.\left.\right|_{\phi}\right|^{2}\right)=0$ est aussi, d'après la décomposition spectrale de $A$, suffisante. On a ainsi obtenu le

Theoreme 16. (1) Si $\phi \in \mathcal{P}$, on peut décomposer $H_{\phi}$ en la somme directe de deux sous-espaces vectoriels invariants

$$
H_{\phi}=H_{\phi}^{p} \oplus H_{\phi}^{c} ;
$$

toute fonction de $H_{\phi}^{p}$ est presque-périodique; toute fonction $\psi$ de $H_{\phi}^{c}$ vérifie

$$
\underset{t}{M}\left[|\psi(t)|^{2}\right]=0 .
$$

(2) On a, de façon unique, $\phi(x)=\phi^{p}(x)+\phi^{c}(x) ; \phi^{p}(x)$ est de type positif et presque périodique; $\phi^{c}(x)$ est de type positif et vérifie

$$
\underset{t}{M}\left[\left|\phi^{c}(t)\right|^{2}\right]=0
$$

(3) Pour qu'une $\phi \in P$ vérifie $M_{t}\left[|\phi(t)|^{2}\right]=0$, il faut et il suffit que $\mathfrak{T}_{\phi}$ ne contienne aucun sous espace vectoriel invariant de dimension finie non nulle.

(4) Toute $\phi \in \mathcal{P}$ presque-périodique est de la forme

$$
\phi(x)=\sum_{i} \lambda_{i} \phi_{i}(x) \quad\left(\lambda_{i}>0, \sum \lambda_{i}=\phi(e)<+\infty\right)
$$

où les $\phi_{i}(x)$ sont des fonctions élémentaires normées associées à des représentations de dimension finie.

\section{CHAPITRE IV}

\section{PROPRIETES DES FONCTIONS DE TYPE POSITIF ET DE CARRE SOMMABLE}

25. Position du problème. Sur un groupe abélien-par exemple sur $R^{1}$-la théorie des fonctions de carré sommable (pour la mesure invariante) est dominée par la transformation de Fourier. Celle-ci repose, comme nous le montrons par ailleurs( $\left.{ }^{25}\right)$, sur le fait fondamental suivant, que nous énonçons dans le cas de $R^{1}$ :

(35) H. Cartan et R. Godement, Analyse harmonique et théorie de la dualité dans les groupes abéliens localement compacts, Ann. Ecole Norm. (1947). 
Soit $f(x)$ une fonction continue, sommable, et de type positif sur $R^{1}$; soit

$$
F(\lambda)=\frac{1}{(2 \pi)^{1 / 2}} \int f(x) e^{-i \lambda x} d x
$$

la transformée de Fourier de $f$. Alors $F(\lambda)$ est une fonction continue, sommable, positive et l'on a réciproquement

$$
f(x)=\frac{1}{(2 \pi)^{1 / 2}} \int F(\lambda) e^{i \lambda x} d \lambda .
$$

De là résulte que, si $f, g \in L$, la fonction $f * g^{\sim}$-qui est une combinaison linéaire de fonctions continues, sommables et de type positif-a pour transformée de Fourier une fonction continue, sommable (et bornée, donc de carré sommable), qui est évidemment $F(\lambda) \overline{G(\lambda)}$ où $F$ et $G$ sont les transformées de $f$ et $g$; l'on a inversement

$$
f * g^{\sim}(x)=\int F(\lambda) \overline{G(\lambda)} e^{i \lambda x} d \lambda .
$$

En particulier, pour $x=0$, il vient

$$
\int f(x) \overline{g(x)} d x=\int F(\lambda) \overline{G(\lambda)} d \lambda:
$$

par suite

$$
\|f\|_{2}=\|F\|_{2}
$$

en sorte que la transformation est prolongeable à tout $L^{2}$ : ce qui constitue le théorème de Plancherel.

Bien entendu, il n'est pas question de faire ici une théorie de la transformation de Fourier sur un groupe quelconque. A supposer qu'une telle entreprise soit possible, elle exigera la solution préalable de nombreux problèmes, en particulier ceux que nous avons posés au chapitre II.

Mais il n'est peut être pas inutile d'entreprendre une étude systématique des fonctions de carré sommable sur un groupe quelconque et d'étendre à ce cas général un certain nombre de propriétés-plus ou moins explicitement connues d'ailleurs-qui, dans le cas abélien, découlent de la transformation de Fourier; ces propriétés sont les suivantes:

(1) Si $f(x)$ est continue, de carré sommable et de type positif, il existe une fonction $g(x)$ de carré sommable telle que

$$
f(x)=g_{*} g^{\sim}(x)=\int g(x+y) \overline{g(y)} d y
$$

(par la transformation, de Fourier, cette propriété équivaut au fait que la 
transformée de $f$ est sommable);

(2) Si $f(x)$ et $g(x)$ sont de type positif et de carré sommable, la fonction $f * g(x)$ est continue de type positif; en particulier

$$
f * g(0)=\int f(x) \overline{g(x)} d x \geqq 0 ;
$$

(3) Il existe dans $L^{2}$ des fonctions (nécessairement de type positif) telles que

$$
\phi(x)=\phi * \phi^{\sim}(x)
$$

(ce sont celles dont la transformée de Fourier est presque partout égale à 0 ou à 1). De plus, les combinaisons linéaires à coefficients positifs de telles fonctions permettent d'approcher dans $L^{2}$ toute fonction de carré sommable et de type positif.

J'ignore si les résultats présentés dans ce chapitre feront avancer réellement la théorie des groupes non abéliens $\left({ }^{(36}\right)$; il est fort possible qu'ils soient simplement "curieux"! Ils constituent en tous cas une étude systématique (je ne dis pas complète) du produit de composition dans $L^{2}$, et un exemple de ce qu'on peut tirer de la théorie des opérateurs hermitiens et de celle des relations d'ordre.

26. Opérateurs de composition dans $L^{2}$. Dans l'espace de Hilbert $L^{2}$ construit sur la mesure invariante à gauche $d x$, on notera

$$
\|f\|_{2}=\left\{\int|f(x)|^{2} d x\right\}^{1 / 2}, \quad[f, g]=\int f(x) \overline{g(x)} d x
$$

la norme et le produit scalaire. Les identités

$$
\int\left|f\left(s^{-1} x\right)\right|^{2} d x=\int|f(x)|^{2} d x=\int\left|f(x s)(\rho(s))^{1 / 2}\right|^{2} d x
$$

permettent d'y introduire deux représentations unitaires "canoniques" de $G$, à savoir

$$
U_{s} f(x)=f\left(s^{-1} x\right), \quad V_{s} f(x)=f(x s)(\rho(s))^{1 / 2},
$$

dont on voit sans difficulté la continuité.

Soir $\mu$ une mesure de type positif $\operatorname{sur} G$. Si $f, g \in L$ on aura (formule analogue à (I 15'), p. 29):

$$
f * \mu * g^{\sim}(e)=\iint f\left(x y^{-1}\right) \overline{g(x)} \frac{d \mu(y)}{(\rho(y))^{1 / 2}} d x=\int\left[V_{y^{-1}} f, g\right] d \mu(y)
$$

${ }^{(36)}$ On peut toutefois en déduire aisément une partie des relations d'orthogonalité de $\mathrm{V}$. Bargmann. Cf. C. R. Acad. Sci. Paris t. 225 (1947). Précisons que l'hypothèse $d x=d\left(x^{-1}\right)$ a été oublié dans l'énonce du Théorème de la Note II, mais qu'elle semble être indispensable. 
1948]

LES FONCTIONS DE TYPE POSITIF

67

d'où

$$
f * \mu * g^{\sim}(e)=\int\left[f, V_{x} g\right] d \mu(x)
$$

En particulier, suppons $\mu$ de la forme

$$
d \mu(x)=\phi(x) \frac{d x}{(\rho(x))^{1 / 2}} \quad \text { où } \quad \phi \in P^{2}=P \cap L^{2} ;
$$

on aura alors

$$
\begin{aligned}
f * \phi * g^{\sim}(e) & =\iint \phi\left(x^{-1} y\right) f(x) \overline{g(y)} d x d y=\int f(x) d x \int \phi\left(x^{-1} y\right) \overline{g(y)} d y \\
& =\int \overline{g(y)} d y \int \overline{\phi\left(y^{-1} x\right)} f(x) d x
\end{aligned}
$$

et donc (IV 1) se complète par

(IV 2)

$$
\begin{aligned}
f * \phi * g^{\sim}(e) & =\int f(x)\left[U_{x} \phi, g\right] d x=\int\left[f, U_{x} \phi\right] \overline{g(x)} d x \\
& =\int\left[f, V_{x} g\right] \phi(x) \frac{d x}{(\rho(x))^{1 / 2}} .
\end{aligned}
$$

Remarques. (1) $P^{2}$ est l'ensemble des $\phi \in P$ qui sont de carré sommable pour la mesure invariante $a$ gauche $d x$; mais c'est aussi l'ensemble des $\phi \in P$ qui possèdent la même propriété relativement à la mesure invariante d droite $d x / \rho(x)$; car

$$
\phi\left(x^{-1}\right)=\overline{\phi(x)}
$$

entraine

$$
\int|\phi(x)|^{2} d x=\int\left|\phi\left(x^{-1}\right)\right|^{2} \frac{d x}{\rho(x)}=\int|\phi(x)|^{2} \frac{d x}{\rho(x)} .
$$

On notera $P^{2}=P \cap L^{2}$ l'ensemble des fonctions continues de $P^{2}$.

(2) Si $\phi \in P^{2}$ et si $f \in L^{2}$, on a

$$
f * \phi(x)=\int f(x y) \phi\left(y^{-1}\right) d y=\int f(x y) \overline{\phi(y)} d y
$$

et par suite

$$
f * \phi(x)=\left[f, U_{x} \phi\right] .
$$

Si $f \in L$ (ou même si $f \in L^{1} \cap L^{2}$ ) le lemme 7 (p. 12) prouve que $f * \phi \in L^{2}$. 
Alors, pour $f, g \in L,(I V 2)$ s'écrit encore

$$
f * \phi * g \sim(e)=[f, g * \phi]=[f * \phi, g] .
$$

Opérateur associé à une mesure bornée de type positif. On a vu au lemme 6 (p. 12) que si $f \in L^{2}$ et si $\mu$ est bornée, on a $f * \mu \in L^{2}$ et même

$$
\|f * \mu\|_{2} \leqq\|\mu\|_{1} \cdot\|f\|_{2} \text {. }
$$

Nous désignerons par $A_{\mu}$ l'opérateur borné ainsi défini dans $L^{2}: A_{\mu} f=f * \mu$. Il est clair qu'on a

$$
f * \mu * g \sim(e)=\int f * \mu(x) \overline{g(x)} d x=[f * \mu, g]
$$

et en particulier

$$
f * \mu * f^{\sim}(e)=\left[A_{\mu} f, f\right] .
$$

D'où immédiatement la

Proposition 11. Pour qu'une mesure bornée $\mu$ soit de type positif sur $G$, il faut el il suffit que l'opérateur $A_{\mu}$ associé dans $L^{2}$ soit hermitien positif.

La formule donnant $f * \nu$ dans le lemme 6 met en évidence, sans qu'il soit utile d'insister, le fait fondamental que les $A_{\mu}$ sont permutables aux $U_{x}$.

Ces faits valent en particulier si

$$
d \mu(x)=\phi(x) \frac{d x}{(\rho(x))^{1 / 2}}
$$

où $\phi$ est sommable pour la mesure $d x /(\rho(x))^{1 / 2}$; l'ensemble des $\phi \in P$ qui possèdent cette propriété sera noté $P^{1}$; de même, $P^{1}$ sera l'ensemble des fonctions continues de $P^{1}$. On observera que $P^{1}$ est une partie de l'espace $E$ défini au chap. II (p. 39). On se servira donc ici encore de la notation

$$
\|\phi\|=\int|\phi(x)| \frac{d x}{(\rho(x))^{1 / 2}} .
$$

$\left(\|\phi\|\right.$ n'est autre que la norme de la mesure $\phi(x) d x /(\rho(x))^{1 / 2}$.) Une propriété qui n'est pas évidente si $\rho(s) \not \equiv 1$ est la

Proposition 12. Toute fonction continue de type positif, sommable pour $d x /(\rho(x))^{1 / 2}$, est de carré sommable pour $d x$ (c'est d̀ dire: $\left.\mathcal{P}^{1} \subset \mathcal{P}^{2}\right)$.

En effet, notons $A_{\phi}$ l'opérateur associé à la mesure $\phi(x) d x /(\rho(x))^{1 / 2}$. On a évidemment

$$
A_{\phi * \phi}=A_{\phi}^{2}
$$

et comme la norme de $A_{\phi}$ est majorée par $\|\phi\|$, on voit que l'opérateur 


$$
\|\phi\| \cdot A_{\phi}-A_{\phi * \phi}
$$

est hermitien positif. La fonction continue

$$
\psi(x)=\|\phi\| \cdot \phi(x)-\phi * \phi(x)
$$

est donc de type positif, d'où résulte, en écrivant $\psi(e) \geqq 0$, que

$$
\|\phi\|_{2}^{2}=\phi * \phi(e) \leqq\|\phi\| \cdot \phi(e)
$$

d'où la proposition:

Opérateur associé à une $\phi \in P^{2}$. Si $\phi$ est un élément quelconque de $P^{2}$, la fonction $f * \phi$ est dans $L^{2}$ si $f \in L$-mais non nécéssairement si $f$ est quelconque dans $L^{2}$. Toutefois l'opérateur

$$
f \rightarrow f * \phi
$$

défini sur le sous-espace partout dense $L$ de $L^{2}$ est symétrique et positif (d'après (IV 4)) et bien entendu permutable aux $U_{x}$. Les procédés classiques de prolongement $\left.{ }^{(27}\right)$ permettent alors d'obtenir le résultat suivant (dont nous laissons la démonstration au lecteur):

Proposition 13. A toute $\phi \in P^{2}$ est associe un opérateur autoadjoint positif $A_{\phi}$, défini dans un domaine $D_{\phi}$ partout dense dans $L^{2}$, contenant $L$, et invariant par les $U_{x} ;$ si $f \in \mathcal{D}_{\phi}$ on $a$

$$
A_{\phi} f(x)=f * \phi(x)=\left[f, U_{x} \phi\right] .
$$

Naturellement, si $\phi \in P^{1}, A_{\phi}$ est borné et coincide avec l'opérateur précédemment défini.

Elements bornés de $P^{2}$. Nous dirons que $\phi$ est un élément borné de $P^{2}$ si l'opérateur associé $A_{\phi}$ est borné au sens habituel.

On a à ce sujet la

Proposition 14. Toute $\phi \in P^{2}$ est limite forte (au sens de $L^{2}$ ) d'éléments bornês de $P^{2}$.

En effect soit

$$
A_{\phi}=\int_{0}^{+\infty} \lambda d E_{\lambda}
$$

la décomposition spectrale de $A_{\phi}$. $A_{\phi}$ étant permutable aux $U_{x}$, il en est de même des projecteurs $E_{\lambda}$, qui sont donc associés à des variétés $V_{\lambda}$ invariantes par $U_{x}$. Soit $\phi_{\lambda}=E_{\lambda} \phi$ la projection de $\phi$ sur $V_{\lambda}$. Si $f \in V_{\lambda}$ on a

$$
A_{\phi} f(x)=\left[f, U_{x} \phi\right]=\left[E_{\lambda} f, U_{x} \phi\right]=\left[f, U_{x} \phi_{\lambda}\right] ;
$$

si au contraire $f \in L^{2} \theta V_{\lambda}$ on a $\left[f, U_{x} \phi_{\lambda}\right] \equiv 0$ : donc l'opérateur, hermitien

(37) B. von Sz. Nagy, op. cit. pp. 35-36. 
positif et borné, donné par

$$
A_{\lambda}=A_{\phi} \circ E_{\lambda}
$$

est associé à $\phi_{\lambda}$-qui est donc un élement borné de $P^{2}$. Comme $\phi_{\lambda}=E_{\lambda} \phi$ converge fortement vers $\phi$ quand $\lambda \rightarrow+\infty$, la proposition est démontrée. Ajoutons que, comme les $E_{\lambda}$, les $\phi_{\lambda}$ constituent une famille "croissante" dans $P^{2}$-c'est à dire que ${ }^{(38)}$

$$
\lambda \leqq \mu \text { entraine } \phi_{\lambda} \ll \phi_{\mu} .
$$

27. Algèbre des éléments bornés de $P^{2}$. Si $\phi, \psi$ sont deux éléments bornés de $P^{2}$, on a $\phi * \psi \in L^{2}$ (puisque $=A_{\psi} \phi$ ).

On peut se demander à quelle condition on a en outre $\phi * \psi \in P^{2}$. Une condition nécessaire est que l'on ait $\phi * \psi\left(x^{-1}\right)=\overline{\phi * \psi(x)}$, ce qui se traduit immédiatement par $\phi * \psi=\psi * \phi$. Si cette condition est remplie, on a alors pour $f \in L$

$$
\begin{aligned}
A_{\phi} \circ A_{\psi} f(x) & =\left[A_{\psi} f, U_{x} \phi\right]=\left[f, U_{x} A_{\psi} \phi\right]=\left[f, U_{x} \phi * \psi\right] \\
& =\left[f, U_{x} \psi * \phi\right]=\left[A_{\phi} f, U_{x} \psi\right]=A_{\psi} \circ A_{\phi} f(x)
\end{aligned}
$$

donc: $A_{\phi} \circ A_{\psi}$, produit de deux hermitiens positifs bornés permutables, est lui-même hermitien positif borné. D'où la

Proposition 15. $\phi$ et $\psi$ étant deux éléments bornés de $P^{2}$, la condition nécessaire et suffisante pour qu'il en soit de même de $\phi * \psi$ est que $\phi * \psi=\psi * \phi$; on a alors

$$
A_{\phi * \psi}=A_{\phi} \circ A_{\psi} .
$$

Si $\phi$ et $\psi$ vérifient la condition précédente (nous dirons alors que $\phi$ et $\psi$ sont permutables), $\phi * \psi$, étant évidemment continue d'après

$$
\phi * \psi(x)=\left[\phi, U_{x} \psi\right]
$$

est non seulement dans $P^{2}$, mais aussi dans $\mathcal{P}^{2}$. D'où résulte

$$
\phi * \psi(e)=[\phi, \psi] \geqq 0 .
$$

En particulier, soient $\phi$ et $\psi$ deux éléments bornés de $P^{2}$, vérifiant

$$
\phi * \psi=\psi * \phi,
$$

$\phi \ll \psi$.

Alors on a évidemment

$$
\phi * \phi \ll \phi * \psi \ll \psi * \psi
$$

d'où pour $x=e$

(38) D'où résulte que, si $\phi \in \mathcal{P}^{2}$ est elementaire, le spectre de $A_{\phi}$ (au sens ordinaire) se reduit à un point $\lambda(0<\lambda<+\infty)$, et que $\phi * \phi=\lambda \phi$ : ce qui conduit aux relations d'orthogonalité de $\mathrm{V}$. Bargmann déja signalées. 
(IV 5)

$$
\|\phi\|_{2}^{2} \leqq[\phi, \psi] \leqq\|\psi\|_{2}^{2}
$$

De plus on a

$$
\|\psi-\phi\|_{2}^{2}=[\psi-\phi, \psi-\phi]=\|\phi\|^{2}+\|\psi\|^{2}-2[\phi, \psi]
$$

et (IV 5) donne alors

$$
\|\psi-\phi\|_{2}^{2} \leqq\|\psi\|_{2}^{2}-\|\phi\|_{2}^{2}
$$

On déduit de là la

Proposition 16. Soit, dans l'ensemble des éléments bornés de $P^{2}$, un ensemble ordonné filtrant croissant d'éléments $\phi_{\alpha}$ deux à deux permutables. Une condition necessaire et suffisante pour que cet ordonné converge fortement est que $\sup _{\alpha}\left\|\phi_{\alpha}\right\|^{2}$ $<+\infty$.

Démonstration (a) Rappelons que:

(1) $\phi_{\alpha}$ est filtrant croissant si, quels que soient $\alpha, \beta$ il existe $\gamma$ avec $\phi_{\gamma} \gg \phi_{\alpha}$ et $\phi_{\gamma} \gg \phi_{\beta}$;

(2) $\phi_{\alpha}$ converge fortement vers $\phi$ si à tout $\epsilon>0$ correspond un indice $\alpha$ tel que

$$
\phi_{\beta} \gg \phi_{\alpha} \text { entraine }\left\|\phi-\phi_{\beta}\right\|_{2}<\epsilon .
$$

Naturellement, le critère de Cauchy vaut ici: on peut remplacer 2 par

$\left(2^{\prime}\right) \phi_{\alpha}$ converge fortement si, à tout $\epsilon>0$, correspond un indice $\alpha$ tel que

$$
\left.\begin{array}{l}
\phi_{\beta} \gg \phi_{\alpha} \\
\phi_{\gamma} \gg \phi_{\alpha}
\end{array}\right\} \quad \text { entraine }\left\|\phi_{\beta}-\phi_{\gamma}\right\|_{2}<\epsilon .
$$

(b) Ceci étant, la condition $\sup _{\alpha}\left\|\phi_{\alpha}\right\|_{2}<+\infty$ est évidemment nécessaire d'après (IV 5). Supposons la vérifiée, et soit

$$
d=\sup _{\alpha}\left\|\phi_{\alpha}\right\|_{2}
$$

$\epsilon>0$ étant donné, il existe un indice $\alpha$ avec

$$
d^{2}-\epsilon \leqq\left\|\phi_{\alpha}\right\|_{2}^{2} \leqq d^{2}
$$

Si alors $\phi_{\beta} \gg \phi_{\alpha}$ et $\phi_{\gamma} \gg \phi_{\alpha}$, on aura d'après (IV 5)

$$
d^{2}-\epsilon \leqq\left\|\phi_{\beta}\right\|_{2}^{2} \leqq d^{2}, \quad d^{2}-\epsilon \leqq\left\|\phi_{\gamma}\right\|_{2}^{2} \leqq d^{2} ;
$$

puis d'après (IV 6)

$$
\left\|\phi_{\beta}-\phi_{\alpha}\right\|_{2}^{2} \leqq\left\|\phi_{\beta}\right\|_{2}^{2}-\left\|\phi_{\alpha}\right\|_{2}^{2} \leqq 2 \epsilon \text { et }\left\|\phi_{\gamma}-\phi_{\alpha}\right\|_{2}^{2} \leqq 2 \epsilon,
$$

d'où 


$$
\left\|\phi_{\beta}-\phi_{\gamma}\right\|_{2}^{2} \leqq 4 \epsilon:
$$

ce qui prouve la proposition puisque le critère de Cauchy est vérifié.

Bien entendu, la limite $\phi$ des $\phi_{\alpha}$ est dans $P^{2}$-mais n'en est pas necéssairement un élément borné.

Il est au surplus à peu près immédiat de voir que $\phi \gg \phi_{\alpha}$ pour tout $\alpha$, et que

$$
f * \phi * f \sim(e)=\sup _{\alpha} f * \phi_{\alpha} * f^{\sim}(e) \quad \text { pour toute } f \in L .
$$

28. Racine carrée d'une fonction de $\mathcal{P}^{2}$. $\phi$ étant un élément borné de $P^{2}$, l'opérateur $A_{\phi}$-qu'on peut supposer $\leqq 1-$ a une racine carrée positive $B$ qu'on obtient de la façon suivante $\left({ }^{(39)}\right.$ :

On pose

$$
B_{0}=0, \cdots, B_{n+1}=B_{n}+\left(A_{\phi}-B_{n}^{2}\right) / 2
$$

alors les $B_{n}$ sont hermitiens positifs $\leqq 1$, deux à permutables, et vont en crolssant: ils ont donc une borne supérieure $B$-qui vérifie $B^{2}=A_{\phi}$.

Mais la proposition 14 nous permet de définir corrélativement les éléments bornés suivants de $P^{2}$ :

$$
\phi_{0}=0, \cdots, \phi_{n+1}=\phi_{n}+\left(\phi-\phi_{n} * \phi_{n}\right) / 2 ;
$$

il est trivial que

$$
B_{n}=A_{\phi_{n}}:
$$

les $\phi_{n}$ constituent donc une suite croissante d'éléments deux à deux permutables; de plus on a

$$
B_{n}^{2} \leqq A_{\phi}
$$

donc

$$
\phi_{n} * \phi_{n} \ll \phi .
$$

Comme les $\phi_{n} * \phi_{n}$ vérifient alors les conditions de la proposition 16, on en conclut qu'ils ont une borne supérieure-nécéssairement égale à $\phi$ puisque

$$
A_{\phi}=\sup A_{\phi_{n^{*} \phi_{n}}} \text {. }
$$

Remarquons maintenant que $\phi_{n} * \phi_{n} \in \mathcal{P}^{2}$ : nous avons la

Proposition 17. Tout élément borné de $P^{2}$ est limite forte d' une suite croissante d'élements bornés deux à deux permutables de $\mathbf{P}^{2}$.

Supposons maintenant que, pour un voisinage $V$ de l'unité, on ait

(39) B. von Sz. Nagy, op. cit. 


$$
|\phi(x)| \leqq M<+\infty
$$

Alors on a $\phi_{n} * \phi_{n}(e) \leqq M$ c'est à dire

$$
\left\|\phi_{n}\right\|_{2}^{2} \leqq M
$$

et d'après la proposition 15 la suite $\phi_{n}$ elle-même converge vers un élément $\psi \in P^{2}$ tel que

$$
A_{\psi}=\sup A_{\phi_{n}}=B \text { : }
$$

d'où

$$
A_{\phi}=A_{\psi}^{2} \quad \text { c'est a dire } \phi=\psi * \psi,
$$

et donc $\phi(x)$ est presque partout égal à la fonction continue $\psi * \psi(x)$. On retrouve ainsi la proposition 4 dans un cas particulier-et on a en même temps démontré que toute $\phi$ bornée de $\mathcal{P}^{2}$ est de la forme $\psi * \psi(x)$, où $\psi$ est un élément bornée de $P^{2}$. Soit maintenant une $\phi$ non bornée de $\Phi^{2}$. Soit, comme au $\$ 26$,

$$
A_{\phi}=\int_{0}^{+\infty} \lambda d E_{\lambda}
$$

la décomposition spectrale de $A_{\phi}$; la fonction $\phi_{n}=E_{n} \phi$ de $P^{2}$ est majorée par $\phi$-donc continue-c'est à dire dans $\Phi^{2}$; comme elle est de plus "bornée", on a

$$
\phi_{n}=\psi_{n} * \psi_{n}
$$

où $\psi_{n} \in P^{2}$. Il est clair que, comme les $\phi_{n}$, les $\psi_{n}$ forment une suite croissante et sont deux à deux permutables. De plus on a

$$
\left\|\psi_{n}\right\|_{2}^{2}=\phi_{n}(e) \leqq \phi(e)
$$

et la proposition 16 prouve alors que $\psi_{n}$ converge fortement vers $\psi \in P^{2}$. Par suite

$$
\phi_{n}(x)=\left[\psi_{n}, U_{x} \psi_{n}\right]
$$

converge uniformément sur $G$ vers $\psi * \psi$-qui est donc nécéssairement égale à $\phi$ puisque $\phi_{n}$ converge fortement vers $\phi$. Ainsi est prouvé le

Theoreme 17. Toute fonction continue $\phi(x)$, de type positif et de carre sommable, est de la forme

$$
\phi(x)=\psi * \psi \sim(x)=\int \psi(x y) \overline{\psi(y)} d y \quad \text { où } \quad \psi \in P^{2} .
$$

Comme $L$ est dense dans $L^{2}$, en approchant $\psi$ par des $f \in L$ on voit que $\phi$ 
est limite, uniforme sur $G$, de fonctions de la forme $f * \tilde{f}-c^{\prime}$ 'est à dire de fonctions de type positif nulles en dehors de compacts.

Par ailleurs, le théorème 17 s'écrit

$$
\phi(x)=\left[\psi, U_{x} \psi\right]:
$$

on en conclut que la structure unitaire associée à $\phi$ est contenue dans $\left\{L^{2}, U_{x}\right\}$.

Le théorème 17 permet de prouver une inégalité importante:

Proposition 18. Si $\phi$ et $\psi$ sont dans $P^{2}$, on a

$$
\int \phi(x) \psi(x) \frac{d x}{(\rho(x))^{1 / 2}} \geqq 0 .
$$

Démonstration. (a) Posons d'une manière générale

$$
f^{\sim}(x)=f\left(x^{-1}\right) /(\rho(x))^{1 / 2} .
$$

Si $f \in L$ on a $f^{\sim} \in L$, et

$$
\|f\|_{2}=\|f\|_{2}
$$

l'opérateur $f \rightarrow f^{2}$ est donc prolongeable à tout $L^{2}$, et est unitaire.

(IV 7) s'écrit alors $\left[\phi, \psi^{\prime}\right] \geqq 0$, ce qui prouve que le premier membre de (IV 7) a un sens.

(b) Tout élément de $P^{2}$ étant (Proposition 14) limite forte d'éléments bornés de $P^{2}$, on peut se ramener, pour démontrer (IV 7), au cas où $\phi$ et $\psi$ sont euxmêmes des éléments bornés de $P^{2}$. En outre, $\mathscr{P}^{2}$ étant dense dans $P^{2}$ (Proposition 17) on peut supposer $\phi \in \mathbb{P}^{2}$, d'où $\phi=\theta * \theta^{\sim}$, auquel cas (IV 7) se réduit à prouver

$$
\int\left[\theta, U_{x} \theta\right] \psi(x) \frac{d x}{(\rho(x))^{1 / 2}} \geqq 0 .
$$

(c) Observons maintenant qu'on a d'une manière générale, si $f, g \in L^{2}$ :

$$
\left[f, U_{x} g\right]=\left[f^{\sim}, V_{x^{-1}} g^{-}\right] \text {. }
$$

Alors $(\alpha)$ se ramène à

$$
\int\left[V_{x} \theta^{\smile}, \theta^{\smile}\right] \psi(x) \frac{d x}{(\rho(x))^{1 / 2}} \geqq 0,
$$

ou, en remplacant $\psi$ par $\Psi$ (qui est aussi un élément borné de $P^{2}$ ), à

$$
\int\left[\theta^{\smile}, V_{x} \theta^{\smile}\right] \psi(x) \frac{d x}{(\rho(x))^{1 / 2}} \geqq 0 .
$$

En comparant avec (IV 2), la proposition sera donc prouvée si l'on démontre que 


$$
\int\left[\theta^{\curlyvee}, V_{x} \theta^{\smile}\right] \psi(x) \frac{d x}{(\rho(x))^{1 / 2}}=\left[A_{\psi} \theta^{\smile}, \theta^{\smile}\right] .
$$

(d) D'après (IV 2) et (IV 4) on a d'abord

$$
\int\left[f, V_{x} g\right] \psi(x) \frac{d x}{(\rho(x))^{1 / 2}}=\left[A_{\psi} f, g\right]
$$

pour $f, g \in L$. Supposons $g$ fixe $(\in L)$ et faisons tendre $f$ fortement vers $\theta^{\smile}$. $A_{\psi}$ étant borné, le second membre tend vers $\left[A_{\psi} \theta^{-}, g\right]$. Dans le premier, posons

$$
h(x)=\frac{1}{(\rho(x))^{1 / 2}}\left[f, V_{x} g\right]
$$

Alors on a

$$
h^{\smile}(x)=\left[V_{x} f, g\right]=\left[f^{\sim}, U_{x} g^{\smile}\right]=f^{\sim} *\left(g^{\smile}\right)^{\sim}(x) .
$$

Comme $g_{1}=\left(g^{\smile}\right)^{\sim}$ est dans $L$, et que, comme on l'a déja vu, on a

$$
\left\|\sim{ }^{*} g_{1}\right\|_{2} \leqq\left\|g_{1}\right\| \cdot\|\sim\|_{2}
$$

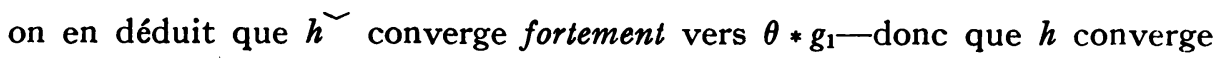
fortement vers

$$
\frac{1}{(\rho(x))^{1 / 2}}\left[\theta^{-}, V_{x} g\right]:
$$

comme $\psi \in L^{2}$, le premier membre tend vers sa valeur pour $f=\theta^{\smile}$, d'où résulte

$$
\int\left[\theta^{\smile}, V_{x} g\right] \psi(x) \frac{d x}{(\rho(x))^{1 / 2}}=\left[A_{\psi} \theta^{\smile}, g\right]
$$

Faisons maintenant tendre $g$ vers $\theta^{`}$. Le second membre tend vers $\left[A_{\downarrow} \theta^{2}, \theta^{-}\right]$; au premier, on a

$$
\left[\theta^{\smile}, V_{x} g\right]=\left[U_{x} \theta, g^{\smile}\right]=\overline{\left[g^{\smile}, U_{x} \theta\right]}=\overline{A_{\theta} g^{\complement}(x)}
$$

en sorte que ce premier membre s'écrit

$$
\left[\psi_{1}, A_{\theta} g^{\smile}\right] \quad \text { où } \quad \psi_{1}(x)=\frac{\psi(x)}{(\rho(x))^{1 / 2}}=\overline{\psi^{\complement}(x)} .
$$

Comme $A_{\theta}$ est borné ${ }^{(40)}$, le premier membre converge donc vers

$$
\left[\psi_{1}, A_{\theta} \theta^{\smile}\right]=\int\left[\theta^{\smile}, V_{x} \theta^{\smile}\right] \psi(x) \frac{d x}{(\rho(x))^{1 / 2}}
$$

${ }^{\left({ }^{40}\right)}$ Rappelons que $\theta$ est la "racine carrée positive" de l'viément borné $\phi$ de $\mathcal{P}^{2}$. 
et on a finalement

$$
\int \phi(x) \psi(x) \frac{d x}{(\rho(x))^{1 / 2}}=\int\left[\theta^{\smile}, V_{x} \theta^{\smile}\right] \psi(x) \frac{d x}{(\rho(x))^{1 / 2}}=\left[A_{\psi} \theta, \theta\right] \geqq 0
$$

ce qui achève de démontrer la proposition.

Un problème d'approximation. Si $\phi$ et $\psi$ appartiennant à $P^{2}$, la fonction $\zeta(x)=\phi(x) \psi(x)$ est, d'après ce qui précède, sommable pour $d x /(\rho(x))^{1 / 2}$; au surplus, elle est de type positif. En effet, si $f \in L$, on a

$$
f * \zeta * f^{\sim}(x)=\int f * f^{\sim}(x) \zeta(x) \frac{d x}{(\rho(x))^{1 / 2}}=\int f * f^{\sim}(x) \cdot \phi(x) \psi(x) \frac{d x}{(\rho(x))^{1 / 2}} ;
$$

comme $f * f^{\sim}$ est une fonction continue de type positif, et que $\phi$ est (Proposition 17) limite forte dans $L^{2}$ de fonctions $\phi_{n}$ continues de type positif, $f * f^{\sim}(x)$ - $\phi(x)$ est limite forte des fonctions continues de type positif $f * f^{\sim}(x) \phi_{n}(x)$ : l'inégalité $f * \zeta * f^{\sim}(e)>0$ s'obtient alors par la proposition 18. On a donc $\zeta \in P^{1}$.

Enfin, $\zeta$ vérifie (Proposition 18) l'inégalité

$$
\int \zeta(x) \frac{d x}{(\rho(x))^{1 / 2}} \geqq 0
$$

On est donc conduit au problème suivant, que je n'ai pu résoudre directement que dans le cas des groupes abéliens:

Problème 4. Si une mesure $\mu$ bornée est de type positif sur $G$, a-t-on( $\left.{ }^{41}\right)$

$$
\int d \mu(x) \geqq 0 \text { ? }
$$

L'importance de ce problème est son équivalence avec le

Probleme 5. Peut-on approcher la constante 1, uniformément sur tout compact, par des fonctions (de type positif) de la forme $f * \tilde{f}(x)$, où $f \in L$ ?

L'équivalence de ces deux problèmes résulte des remarques suivantes:

(1) Supposons qu'on puisse approcher 1 par des $f_{\alpha} * \tilde{f_{\alpha}}$, et ceci uniformément sur tout compact. Alors pour toute mesure bornée $\mu$ de type positif on aura

$$
\int d \mu(x)=\lim _{\alpha} \int f_{\alpha} * f_{\alpha}^{\sim}(x) d \mu(x) \geqq 0 .
$$

(2) Réciproquement, supposons qu'on ait

$$
\int d \mu(x) \geqq 0
$$

(11) Le problème 4 peut encore s'énoncer comme suit: soient $\left\{\mathcal{H C}, U_{x}\right\}$ une structure unitaire $\operatorname{sur} G$, et $\mu$ une mesure bornée de type positif; alors, l'opérateur de Radon $T_{\mu}$ associé à $\mu$ dans $\mathfrak{H C}$ (cf. \$6) est-il hermitien positif? 
pour toute $\mu$ bornée de type positif. On aura en particulier

$$
\int \phi(x) \frac{d x}{(\rho(x))^{1 / 2}} \geqq 0
$$

pour toute $\phi \in P^{1}$. Revenons alors à $\mathcal{P}_{0}, E, E^{\prime}$ (notations du chap. II, §14). Dans $\mathcal{P}_{0}$, les fonctions qui sont de carré sommable (c'est à dire dans $\mathbb{P}^{2}$ ) forment un ensemble convexe $A$; comme $P^{1}$ est l'ensemble des $\phi \in E$ qui vérifient

$$
(\phi, \psi)=\int \phi(x) \psi(x) \frac{d x}{(\rho(x))^{1 / 2}} \geqq 0 \text { pour toute } \psi \in A,
$$

le lemme $B$ (\$13) nous montre alors que la constante 1 est faiblement adhérente $a A$. Il est facile d'en déduire plus généralement qu'alors toute fonction continue de type positif est limite, uniforme sur tout compact, de fonctions (de type positif) de la forme $f * \tilde{f^{\prime}}(f \in L)$.

Il n'est peut être pas superflu d'indiquer que, de toute façon, l'adhérence faible de $A$ constitue une partie régulière de $\mathcal{P}_{0}$. Car si $\phi \in A$, on a $\phi=\psi * \psi$ $\left(\psi \in P^{2}\right.$; cf. Théorème 17). Donc si $\theta \ll \phi$, on a (Théorème 6$) \theta=\zeta * \zeta^{2}$ où $\zeta \in L^{2}$ -et par suite $\theta$ est limite dans $\Phi_{0}$ de fonctions $f * f^{\sim}(f \in L)$. Indiquons une voie par laquelle on pourrait tenter de résoudre la problème 5 : c'est de prouver d'une manière générale que, si une $\phi \in \mathcal{P}$ est en même temps positive au sens habituel, alors son spectre contient la constante 1 . En appliquant alors cette propriété à $\phi=f * \tilde{f}$ où $f \in L_{0}^{+}$, le problème 5 serait résolu. J'ignore si cette hypothèse est vérifiée dans le cas général; il est en tous cas possible de voir qu'elle l'est sur un groupe abélien ou compact.

29. Théorie des unités. Nous appellerons unité de $G$ toute $\phi \in P^{2}$ telle que

$$
\phi(x)=\phi * \phi(x)
$$

l'opérateur associé $A_{\phi}$ est alors un projecteur. L'existence de telles fonctions sur un groupe quelconque résulte de la

Proposition. 19. Soit $\psi$ un élément borné de $P^{2}$, et $E$ un projecteur dans $L^{2}$ tel que

(1) $E \leqq A_{\psi}\left({ }^{42}\right)$.

(2) Tout opérateur borné permutable à $A_{\psi}$ permute à $E$. Alors $E=A_{\phi}$ où $\phi$ est une unité.

Démonstration. Soit $\Phi$ la famille des éléments bornés $\theta \in P^{2}$ qui vérifient

$(\alpha)$ Tout opérateur borné permutable à $A_{\psi}$ permute à $A_{\theta}$;

(B) $E \leqq A_{\theta}$.

$\Phi$ n'est pas vide, car $\psi \in \Phi$. De plus, les $\theta \in \Phi$ sont, d'après $(\alpha)$, deux à deux

(12) Cette condition exprime que $E$ appartient à l' "anneau d'opérateurs" endendré par $A_{\psi}$. 
permutables, et les $A_{\theta}$ permutent à $E$. Soient maintenant deux éléments $\theta_{1}, \theta_{2} \in \Phi:$ je dis qu'il existe un $\theta_{3} \in \Phi$ avec $\theta_{3} \ll \theta_{1}, \theta_{3} \ll \theta_{2}$ - 'est à dire que $\Phi$ est un ordonné filtrant décroissant. Soit en effet $B=A_{\theta_{1}}-A_{\theta_{2}}$; désignons par $V^{+}$la "plus grande variété" sur laquelle $B$ est $\geqq 0$, et par $V^{-}$la variété supplémentaire. $V^{+}$et $V^{-}$sont naturellement invariantes par les $U_{x}$, et si $f \in V^{+}$on a

$$
B f(x)=\left[f, U_{x} \theta_{1}\right]-\left[f, U_{x} \theta_{2}\right]=\left[f, U_{x} \xi\right]
$$

où $\xi$ est la projection orthogonale de $\theta_{1}-\theta_{2}$ sur $V^{+}$. Comme $B$ est $\geqq 0$ sur $V^{+}$, il en résulte que $\xi \in P^{2}$ et que

$$
A_{\xi}=\left\{\begin{array}{lll}
B & \text { sur } & V^{+} \\
0 & \text { sur } & V^{-}
\end{array}\right.
$$

De même, si $-\eta$ est la projection de $\theta_{1}-\theta_{2}$ sur $V^{-}$, on a $\eta \in P^{2}$ et

$$
A_{\eta}=\left\{\begin{array}{rll}
0 & \text { sur } & V^{+} \\
-B & \text { sur } & V^{-}
\end{array}\right.
$$

Posons alors

$$
\theta_{3}=\theta_{2}-\eta \quad\left(=\inf \left(\theta_{1}, \theta_{2}\right) !\right):
$$

il est facile de voir que $\theta_{3}$ répond à la question.

Ceci étant, $\Phi$, qui est un ordonné filtrant décroissant d'éléments deux à deux permutables, possède un borne inférieure $\phi$. Evidemment $\phi \in \Phi$ (car les conditions $(\alpha)$ et $(\beta)$ se conservent par passage à la limite forte); et tout $\theta \in \Phi$ "majore" $\phi$. En particulier, comme $E \leqq A_{\phi}$, on a $E \leqq A_{\phi}^{2}$-donc $\phi * \phi \in \Phi$ et par suite

$$
\phi \ll \phi * \phi .
$$

Mais alors $\phi$, majoré par $\phi * \phi$ qui est continue, est lui même dans $P^{2}$-donc de la forme $\phi=\theta * \theta$ où $\theta \in P^{2}$. De $E \leqq A_{\phi}$ résulte $E \leqq A_{\theta}$ : et donc $\theta \in \Phi$; donc $\theta \gg \phi$ et

$$
\phi=\theta * \theta \gg \phi * \phi
$$

comparant (\#) et (\#\#) on obtient $\phi=\phi * \phi: \phi$ est une unité. Reste à prouver que $E=A_{\phi}$. Or on a $E \leqq A_{\phi}$; soit $\phi^{\prime}=E \phi$; on constate sans peine que $\phi^{\prime} \in \Phi-$ donc $\phi^{\prime} \gg \phi-$ mais aussi $\ll \phi\left({ }^{43}\right)$ : donc $\phi^{\prime}=E \phi$, et finalement la proposition 18 se trouve démontrée.

Suites spectrales. Soit $\phi$ un élément de $P^{2}$; soit

$$
A_{\phi}=\int_{0}^{\infty} \lambda d E_{\lambda}
$$

(4) Car on a $A_{\phi^{\prime}}=E A_{\phi}=A_{\phi} E \leqq A_{\phi}$. 
la décomposition spectrale de $A_{\phi}$. Alors le projecteur $F_{\lambda}=1-E_{\lambda}$ vérifie, comme on le sait, l'inégalité

$$
\lambda F_{\lambda} \leqq A_{\phi} .
$$

Si donc $\lambda>0$, on a $F_{\lambda}=A_{\phi_{\lambda}}$ où $\phi_{\lambda}$ est une unité. Il est claịr que les $\phi_{\lambda}$ jouissent des propriétés suivantes:

(1) $\phi_{\lambda}=\phi_{\lambda} * \phi_{\lambda}$;

(2) $\lambda \geqq \mu$ entraine $\phi_{\lambda} \ll \phi_{\mu}$;

(3) $\phi_{\lambda} * \phi_{\mu}=\phi_{\mu} * \phi_{\lambda}=\phi_{\nu}$ où $\nu=\inf (\lambda, \mu)$;

(4) Pour $\lambda \rightarrow \infty, \phi_{\lambda}$ tend vers zéro dans les deux topologies suivantes: convergence forte dans $L^{2}$; convergence uniforme sur $G$.

Il est clair aussi qu'on a

$$
\phi=-\int_{0}^{+\infty} \lambda d \phi_{\lambda}
$$

intégrale à laquelle la structure d'ordre de $P^{2}$ permet de donner un sens précis. Par ailleurs, si l'on subdivise $(0,+\infty)$ en intervalles partiels par des points $\lambda_{i}\left(\lambda_{i}<\lambda_{i+1}\right)$, et si l'on pose

$$
e_{i}=\phi_{\lambda_{i}}-\phi_{\lambda_{i+1}},
$$

$e_{i}$ est une unité et on parvient sans peine à la

Proposition 20. Toute $\phi \in P^{2}$ est limite forte de fonctions de la forme $\sum \lambda_{i} e_{i}(x)$ où les $e_{i}$ sont des unités permutables à $\phi$, et les $\lambda_{i}$ des nombres $>0$.

Application aux groupes compacts. Les résultats précédents permettent de retrouver aisément le théorème de Peter-Weyl pour les groupes compacts.

Observons tout d'abord que, si $G$ est compact, les opérateurs $A_{\phi}$ sont completement continus. En effet, si $f \in L^{2}$, on a

$$
A_{\phi} f(x)=\left[f, U_{x} \phi\right]
$$

$G$ étant compact, et l'application $x \rightarrow U_{x} \phi$ de $G$ dans $L^{2}$ fortement continue, les $U_{x} \phi$ forment une partie $\mathfrak{I}_{\phi}$ fortement compacte de $L^{2}$. Si alors $f$ converge faiblement dans $L^{2}$ vers $f_{0}$ en restant dans une sphère fixe, la fonction $A_{\phi} f(x)$ converge vers $A_{\phi} f_{0}(x)$ uniformément sur $\mathfrak{T}_{\phi}$ - 'est à dire sur $G$ (Lemme de Gelfand déja cité)—et donc fortement dans $L^{2}$, ce qui prouve la complète continuité (on notera aussi que, ici, tous les $\phi \in P^{2}$ sont bornés).

Ceci étant, soit $e$ une unité: $A_{e}$ est un projecteur complètement continudonc de dimension finie; par suite, $e$ est une somme finie de fonctions élémentaires. La proposition 20 prouve alors que les combinaisons linéaires finies de fonctions élémentaires sont denses dans $P_{2}$, donc dans $L_{2}$, et on en déduit sans peine le résultat en question de Péter-Weyl. Naturellement, la présente méthode n'a rien d'original: elle se trouve dans le travail de ces deux auteurs et, sous une forme plus systématique, dans l'ouvrage d'André Weil. 


\section{APPENDICE}

Cet appendice (écrit en novembre 1947 pendant la correction des épreuves) a pour but de complèter les résultats précédents sur quelques points, et d'indiquer leurs relations avec ceux d'autres auteurs-parus après la rédaction de ma Thèse.

\section{A. SUR LES REPRESENTATIONS UNITAIRES IRREDUCTIBLES DES GROUPES DISCRETS}

Soient $\Omega$ un ensemble infini, et $G$ un groupe de permutations de $\Omega$; on désignera par $s, t, \ldots$ les éléments de $G$; $\operatorname{par} \alpha, \beta, \cdots$ ceux de $\Omega$; et par $s \alpha$ le transformé d'un $\alpha \in \Omega$ par un $s \in G$. $\Omega$ et $G$ seront considérés comme des espaces topologiques discrets (c'est-à-dire, en fait, comme des ensembles dénués de topologie).

Désignons par 3 l'espace.de Hilbert (non séparable si $\Omega$ n'est pas dénombrable) formé par les fonctions numériques complexes $f(\alpha)$ définies sur $\Omega$ et vérifiant

$$
\sum_{\alpha \in \Omega}|f(\alpha)|^{2}<+\infty .
$$

Les fonctions

$$
e_{\alpha}(\beta)=\left\{\begin{array}{lll}
1 & \text { si } & \beta=\alpha, \\
0 & \text { si } & \beta \neq \alpha
\end{array}\right.
$$

constituent évidemment une base orthonormale de $\mathfrak{T C}$.

Une function numérique complexe $\mu(s, \alpha)$ définie sur $G \times \Omega$ sera un multiplicateur du groupe de transformations $G$ si elle possède les deux propriétés suivantes:

$$
\begin{array}{r}
|\mu(s, \alpha)|=1 \quad \text { quels que soient } s \in G, \alpha \in \Omega, \\
\mu(s t, \alpha)=\mu(s, \alpha) \mu\left(t, s^{-1} \alpha\right) \text { pour } s, t \in G, \alpha \in \Omega .
\end{array}
$$

Tout multiplicateur permet de définir une représentation unitaire de $G$ dans $\Re$, obtenue en posant

$$
U_{s}^{\mu} f(\alpha)=\mu(s, \alpha) f\left(s^{-1} \alpha\right) .
$$

Il est clair qu'on a

$$
U_{s}^{\mu} e_{\alpha}=\mu(s, s \alpha) e_{s \alpha}
$$

THEOREME. Supposons réalisées les conditions suivantes:

(a) $G$ est transitif dans $\Omega$;

(b) Si $\beta \neq \alpha$, les s $\beta$ où s vérifie s $\alpha \times \alpha$, forment un sous-ensemble infini de $\Omega$; alors la représentation unitaire $s \rightarrow U_{s}^{\mu}$ de $G$ dans $\mathfrak{H}$ est irréductible. 
Démonstration. Il suffit de prouver que tout opérateur linéaire borné $A$ permutable aux $U_{s}^{\mu}$ est un multiple de l'unité. Or, pour un tel $A$, on aura des équations de la forme

$$
A e_{\alpha}=\sum_{\beta} a(\alpha, \beta) e_{\beta}
$$

avec

$$
\sum_{\beta}|a(\alpha, \beta)|^{2}<+\infty .
$$

En écrivant que $A U_{s}^{\mu} e_{\alpha}=U_{s}^{\mu} A e_{\alpha}$, on trouve immédiatement

$$
a(s \alpha, \beta) \mu(s, s \alpha)=a\left(\alpha, s^{-1} \beta\right) \mu(s, \beta),
$$

d'où résulte d'après (1)

$$
\left|a\left(\alpha, s^{-1} \beta\right)\right|^{2}=|a(\alpha, \beta)|^{2} \quad \text { pour } s \alpha=\alpha .
$$

Si donc $\beta \neq \alpha$, la série (5) comprendra-condition (b)-une infinité de termes égaux à $|a(\alpha, \beta)|^{2}$ : on a donc

$$
a(\alpha, \beta)=0
$$

pour $\beta \neq \alpha$.

Par ailleurs, $(6)$ donne $(\beta=s \alpha)$ :

$$
a(s \alpha, s \alpha) \mu(s, s \alpha)=a(\alpha, \alpha) \mu(s, s \alpha)
$$

d'où

$$
a(\beta, \beta)=a(\alpha, \alpha)=\lambda \quad \text { quels que soient } \alpha, \beta \in \Omega
$$

puisque l'on peut toujours-condition (a)-écrire $\beta=s \alpha$. Donc $A e_{\alpha}=\lambda e_{\alpha}$ pour tout $\alpha$, et le théorème est démontré.

Exemple 1. $\Omega=Q$ corps des nombres rationnels; $G$ : transformations

$$
s(a, b): \quad \alpha \rightarrow a \alpha+b
$$

où $a, b \in Q, a \neq 0$; multiplicateurs: soit $\chi$ un caractère du groupe multiplicatif des rationnels $\neq 0$-par exemple $\chi(a)=|a|^{i \sigma}, \sigma$ réel-il suffit alors de prendre

$$
\mu(s, \alpha)=\chi(a) \quad \text { pour } s=s(a, b) .
$$

Naturellement, ce procédé est valable pour tout corps commutatif infini.

Exemple 2. $\Omega=Q_{\infty}$ ensemble des nombres rationnels auquel on ajoute formellement un point $\infty ; G$ : groupe modulaire arithmétique

$$
s(a, b, c, d): \alpha \rightarrow \frac{a \alpha+b}{c \alpha+d} \quad \text { pour } \alpha \cdot \neq \infty ; \infty \rightarrow \frac{a}{c}
$$


avec $a, b, c, d$ entiers rationnels, $a d-b c=1$; le multiplicateur le plus simple est $\mu(s, \alpha) \equiv 1$.

En effectuant une représentation conforme du demi-plan $\Im(z) \geqq 0$ sur le cercle $|\zeta| \leqq 1$, on pourrait représenter $G$ comme sous-groupe du groupe hyperbolique-substitutions $z \rightarrow(a z+b) / \bar{b} z+\bar{a}$ avec $a \bar{a}-b \bar{b}=1$-et le réaliser ainsi comme groupe de permutations d'un sous-ensemble dénombrable et partout dense de la circonférence $|\zeta|=1$; on aurait alors en évidence les multiplicateurs utilisés par V. Bargmann $\left({ }^{44}\right)$ pour le groupe hyperbolique tout entier.

Cet exemple prouve que-contrairement à l'hypothèse émise au \$15un groupe maximalement presque périodique peut aussi possèder des représentations unitaires irréductibles de dimension infinie.

Exemple 3. $\Omega=Q_{\infty} ; G$ : substitutions

$$
\alpha \rightarrow \frac{a \alpha+b}{c \alpha+d}
$$

$a, b, c, d$ rationnels, $a d-b c=1$; multiplicateur: $\mu(s, \alpha)=1$.

On obtiendrait d'autres représentations de ce groupe en le considérant comme sous-groupe partout dense du groupe hyperbolique. On sait par ailleurs que $G$ est minimalement presque-périodique.

Exemple 4. $\Omega=C_{\infty}$, sphère de Riemann (munie de la topologie discrète); $G$ : groupe de Lorentz

$$
\alpha \rightarrow \frac{a \alpha+b}{c \alpha+d},
$$

$a, b, c, d$ complexes, $a d-b c=1$; multiplicateur $\mu(s, \alpha)=1$. On est ainsi conduit à une représentation unitaire irréductible non continue du groupe de Lorentz. On observera qu'ici $3 C$ est non séparable. On pourrait évidemment donner des exemples analogues - et pathologiques-avec la plupart des groupes connus. Il serait, de beaucoup, plus intéressant de découvrir une généralisation topologique du Théorème, de façon à posséder des démonstrations purement topologiques de l'irréductibilité des représentations construites récemment par V. Bargmann et Gelfand et Neumark( $\left.{ }^{45}\right)$. Il est évident que des conditions de transitivité assez fortes doivent jouer un rôle décisif dans ce problème.

On observera que des considérations analogues aux précédentes ont permis à F. J. Murray et J. von Neumann-apparemment, inventeurs de la

(4) V. Bargmann, Irreducible unitary representations of the Lorentz group, Ann. of Math. t. 48 (1947) pp. 568-640.

(45) I. Gelfand et M. Neumark, Unitary representations of the Lorentz group, Journal of Physics, Acad. Sci. URSS. t. 10 (1946) pp. 93-94. Voir aussi des notes ultérieures sur le groupe $S L(n, C)$ et le groupe affine de la droite. 
notion capitale de multiplicateur-de construire explicitement des exemples de "facteurs" dans la théorie des anneaux d'opérateurs. Il est par ailleurs certain que cette dernière théorie mériterait une étude systématique et approfondie dans ses rapports avec la théorie des groupes $\left({ }^{46}\right)$.

\section{B. SUR LE PROBleme 2}

Soit $G$ le groupe modulaire arithmétique (cf. $A$, Exemple 2). Il est bien connu que $G$ possède deux générateurs $s$ et $t$ assujettis à vérifier les conditions $s^{2}=t^{3}=e$. Dans ces conditions, tout couple d'opérateurs unitaires $U$ et $V$ d'un espace de Hilbert $\mathfrak{H}$, vérifiant $U^{2}=V^{3}=1$, conduit à une représentation unitaire de $G$.

Prenons alors pour $\mathcal{H}$ l'espace de dimension 2. Soient $X_{1}, X_{2}$ et $Y_{1}, Y_{2}$ deux bases orthogonales de $\mathfrak{H}$, reliées (par exemple) par

$$
\begin{aligned}
& Y_{1}=X_{1} \cos \theta+X_{2} \sin \theta, \\
& Y_{2}=-X_{1} \sin \theta+X_{2} \cos \theta,
\end{aligned}
$$

et posons

$$
\begin{array}{ll}
U X_{1}=X_{1}, & V Y_{1}=j Y_{1}, \\
U X_{2}=-X_{2}, & V Y_{2}=j^{2} Y_{2},
\end{array}
$$$$
j=e^{2 i \pi / 3}
$$

Il est clair que pour chaque $\theta \neq k \pi / 2$ on aura une représentation unitaire irréductible de $G$. Soit alors

$$
X_{0}=\alpha_{1} X_{1}+\alpha_{2} X_{2} \quad\left(\alpha_{1} \neq 0, \alpha_{2} \neq 0\right) ;
$$

désignons par $x \rightarrow U_{x}^{\theta}$ la représentation unitaire de $G$ définie par les formules (1) et (2). Alors, pour $\theta \neq k \pi / 2$, la fonction de type positif $\left(U_{x}^{\theta} X_{0}, X_{0}\right)$ est élémentaire; quand $\theta$ tend vers 0 , elle converge faiblement vers la fonction non élémentaire $\left(U_{x}^{0} X_{0}, X_{0}\right) \not \equiv 0$.

Le Problème $2, \$ 15$, n'admet donc pas de réponse affirmative-même dans les cas les plus simples. Il existe naturellement une foule de contre-exemples plus compliqués.

Des résultats "normaux" ont été obtenus( $\left.{ }^{47}\right)$ récemment par l'auteur en considérant des groupes $G$ vérifiant la condition suivante: les automorphismes intérieurs de $G$ forment un groupe compact. Les groupes abéliens et les groupes compacts rentrent dans cette catégorie.

${ }^{(46)}$ Voir à ce sujet (outre les articles de J. von Neumann): I. E. Segal, Unitary representations of rings of operators, Bull. Amer. Math. Soc. t. 53 (1947). On remarquera que (1) il n'est pas certain que la méthode de Segal conduise à toutes les représentations unitaires irréductibles du groupe considéré, (2) par contre, dans l'algèbre définie par Segal, tout idéal maximal est engendré par une représentation unitaire irréductible. Cf. la partie $\mathrm{C}$ de cet appendice.

${ }^{(47)}$ R. Godement, Analyse harmonique dans les groupes centraux, C. R. Acad. Sci. Paris t. 225 (1947) pp. 19-21 et 221-223. 


\section{SUR LE SPECTRE D'UNE Fonction DE $L^{\infty}$}

Sur la question de savoir si toute $\phi \in L^{\infty}$ possède un spectre au sens du $\S 18$, nous nous bornerons ici à quelques remarques:

(1) Tout d'abord, on pourrait donner au mot spectre d'autres sens: on peut, par exemple, se borner à considèrer les translatées à gauche, ou à droite, de $\phi$. Les différents spectres qu'on obtiendrait ainsi-s'ils existent-sont évidemment distincts. Il est toutefois remarquable que, dans le cas d'un groupe $G$ compact, les trois spectres ainsi définis conduisent aux mêmes représentations unitaires irréductibles de $G$.

(2) Il n'est pas difficile de voir que la question dépend en grande partie de la suivante: si a est un idéal à gauche maximal et fermé de l'algèbre $L^{1}$, peut-on trouver une représentation unitaire irréductible $\left\{\mathfrak{F}, U_{x}\right\}$, de $G$ et un $X \in \mathfrak{H}$ tels que a soit l'ensemble des $f \in L^{1}$ pour lesquels

$$
T_{f} X=0 \quad\left(T_{f}=\int U_{x} f(x) d x\right) ?
$$

En d'autres termes: peut-on réaliser toute représentation linéaire irréductible (au sens purement algèbrique de ce terme) de $L^{1}$ dans une représentation unitaire irréductible (au sense adopté dans ce travail) de $G$ ? L'importance de ce problème est évidente $\left({ }^{48}\right)$.

(3) Des résultats définitifs dans ce sens-mais qui concernent des algèbres normées quelque peu différentes des algèbres $L^{1}$-ont été obtenus(49) par $I$. Gelfand et $M$. Neumark; voir aussi des résultats plus récents, aussi positifs, et moins restrictifs de D. A. Raikovo( $\left.{ }^{50}\right)$.

Notons qu'un problème analogue - et qui admet, cette fois, une réponse affirmative - se poserait si, au lieu de considérer $L^{1}$, on envisageait l'algèbre d'opérateurs (au sens de J. von Neumann) engendrée dans l'espace $L^{2}$ par les opérateurs

$$
\phi \rightarrow f * \phi \quad\left(\phi \in L^{2}, f \in L^{1}\right) .
$$

\section{BIBLIOGRAPHIE}

Pour les travaux antérieurs à 1940, voir:

1. A. Weil, L'integration dans les groupes topologiques et ses applications (Actualités Scientifiques et Jndustrielle, no. 869), Paris, Hermann, 1940.

2. I. Gelfand et D. Ralkov, Irreducible unitary representations of arbitrary locally bicompact groups, Rec. Math. (Mat. Sbornik) N.S. t. 13 (1943).

(48) Ce problème est aussi posé par I. E. Segal, The group algebra of a locally compact group, Trans. Amer. Math. Soc. t. 61 (1947) pp. 69-105.

(49) I. Gelfand et M. Neumark, On the imbedding of normed rings into the ring of operators in Hilbert space, Rec. Math. (Mat. Sbornik) N.S. t. 12 (1943) pp. 197-212.

${ }^{(50)}$ D. A. Rarkov, To the theory of normed rings with involution, C. R. Acad. Sci. URSS. t. 54 (1946) pp. 387-390.

NANCY, FRANCE. 